NUREG-1350

Vol. 1

\title{
Nuclear Regulatory Commission 1989 Information Digest
}

U.S. Nuclear Regulatory Commission

Office of the Controller

$$
\begin{aligned}
& \text { DO NOT MICROFILM } \\
& \text { COVER }
\end{aligned}
$$




\title{
DO NOT MICROFILM COVER
}

\section{AVAILABILITY NOTICE}

\author{
Avallability of Reference Materials Cited in NRC Publications
}

Most documents cited in NRC publications will be available from one of the following sources:

1. The NRC Public Document Room, 2120 L Street, NW, Lower Lovel, Washington, DC 20555

2. The Superintendent of Documents, U.S. Government Printing Office, P.O. Box 37082, Washington, DC 20013-7082

3. The National Technical Information Service, Springfield, VA 22161

Although the listing that follows represents the majority of documents cited in NRC publications, it is not intended to be exhaustive.

Referenced documents available for inspection and copying for a fee from the NRC Public Document Room include NRC correspondence and internal NRC memoranda; NRC Office of Inspection and Enforcement bulletins, circulars, information notices, inspection and investigation notices; Licensee Event Reports; vendor reports and correspondence; Commission papers; and applicant and licensee documents and correspondence.

The following documents in the NUREG series are available for purchase from the GPO Sales Program: formal NRC staff and contractor reports, NRC-sponsored conference proceedings, and NRC booklets and brochures. Also available are Regulatory Guides, NRC regulations in the Code of Federal Regulations, and Nuclear Regulatory Commission Issuances.

Documents available from the National Technical information Service include NUREG series reports and technical reports prepared by other federal agencies and reports prepared by the Atomic Energy Energy Commission, forerunner agency to the Nuclear Regulatory Commission.

Documents available from public and special technical libraries include ail open literature items, such as books, journal and periodical articles, and transactions. Federal Register notices, federal and state legislation, and congressional reports can usually be obtained from these libraries.

Documents such as theses, dissertations, foreign reports and translations, and non-NRC conference proceedings are available for purchase from the organization sponsoring the publication cited.

Single copies of NRC draft reports are available free, to the extent of supply, upon written request to the Office of Information Resources Management, Distribution Section, U.S. Nuclear Regulatory Commission, Washington, DC 2055.

Copies of industry codes and standards used in a substantive manner in the NRC regulatory process are maintained at the NRC Library, 7920 Norfolk Avenue, Bethesda, Maryland, and are available there for reference use by the public. Codes and standards are usually copy-righted and may be purchased from the originating organization or, if they are American National Standards, from the American National Standards Institute, 1430 Broadway, Now York NY 10018. 


\section{DISCLAIMER}

This report was prepared as an account of work sponsored by an agency of the United States Government. Neither the United States Government nor any agency Thereof, nor any of their employees, makes any warranty, express or implied, or assumes any legal liability or responsibility for the accuracy, completeness, or usefulness of any information, apparatus, product, or process disclosed, or represents that its use would not infringe privately owned rights. Reference herein to any specific commercial product, process, or service by trade name, trademark, manufacturer, or otherwise does not necessarily constitute or imply its endorsement, recommendation, or favoring by the United States Government or any agency thereof. The views and opinions of authors expressed herein do not necessarily state or reflect those of the United States Government or any agency thereof. 


\section{DISCLAIMER}

Portions of this document may be illegible in electronic image products. Images are produced from the best available original document. 


\section{Nuclear Regulatory Commission 1989 Information Digest}

Manuscript Completed: February 1989

Date Published: March 1989

Division of Budget and Analysis

Office of the Controller

U.S. Nuclear Regulatory Commission

Washington, D.C. 20555

\section{MASTER}

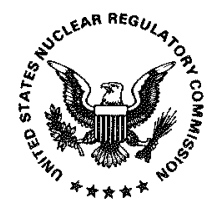

DISTRIBUTION OF THIS DOCUMEAT IS UMLIRITES 


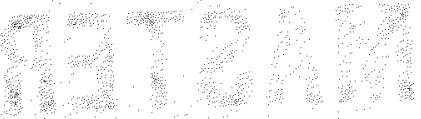




\section{ABSTRACT}

The Nuclear Regulatory Commission 1989 Information Digest provides summary information regarding the U.S. Nuclear Regulatory Commission, its regulatory responsibilities, and areas licensed by the Commission. This is the first of an annual publication for the general use of the NRC staff and is available to the public.

The Digest is divided into two parts: the first presents an overview of the U.S. Nuclear Regulatory Commission and the second provides data on NRC commercial nuclear reactor licensees and commercial nuclear power reactors worldwide.

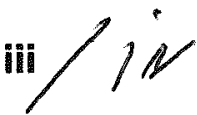




\section{TABLE OF CONTENTS}

\section{PART I: NRC OVERVIEW}

MISSION

STATUTORY AUTHORITY

MAJOR ACTIVITIES

ORGANIZATION

Exhibit 1, U.S. Nuclear Regulatory Commission

GEOGRAPHIC LOCATIONS

Exhibit 2, U.S. NRC Geographic Locations

RESOURCE PROFILES

Exhibit 3, NRC FY 1989 Staff By Location

Exhibit 4, NRC FY 1989 Staff By Function

Exhibit 5, NRC FY 1989 Resources By Mission Area

Exhibit 6, NRC Total Obligations in Constant Dollars (FY 1975-1989)

NUCLEAR POWER REACTORS

Exhibit 7.1, U.S. Commercial Nuclear Power Reactor Sites

Exhibit 7.2, NRC Region I Commercial Reactor Sites

Exhibit 7.3, NRC Region II Commercial Reactor Sites

Exhibit 7.4, NRC Region III Commercial Reactor Sites

Exhibit 7.5, NRC Region IV Commercial Reactor Sites

Exhibit 7.6, NRC Region V Commercial Reactor Sites 


\section{TABLE OF CONTENTS}

Exhibit 8.1, 1987 U.S. Electric Net Generation By Energy Source

Page

Exhibit 8.2, U.S. Electric Net Generation By Nuclear Reactors In Commercial Operation (CY 1976-1988)

Exhibit 8.3, Average Annual U.S. Nuclear Reactor Capacity Factor

Exhibit 9.1, 1987 World Energy Production By Power Source

Exhibit 9.2, 1987 World Nuclear Power Production By Reactors in Commercial Operation

Exhibit 9.3, World Net Nuclear Electric Power Production

Exhibit 10.1, U.S. Commercial Power Reactors By Age/Population

Exhibit 10.2, U.S. Commercial Power Reactors By Age/Electric Generation

Exhibit 10.3, U.S. Commercial Power Reactors By License Expiration Date

NONPOWER NUCLEAR REACTORS

Exhibit 11, Nonpower Nuclear Reactor Sites 35

\section{FUTURE REACTOR DESIGNS}

NUCLEAR MATERIAL SAFETY

Exhibit 12, Major Fuel Cycle Facilities In The U.S.

Exhibit 13, Agreement States

NUCLEAR MATERIAL TRANSPORTATION AND SAFEGUARDS

HIGH LEVEL NUCLEAR WASTE DISPOSAL

Exhibit 14.1, Spent Fuel Stored At Nuclear Power Plants By State 


\section{TABLE OF CONTENTS}

LOW LEVEL NUCLEAR WASTE DISPOSAL

Exhibit 15, Low Level Radioactive Waste By Source

Page

Exhibit 16, Commercial Low Level Waste Disposal Sites

Exhibit 17, Regional Low Level Waste Compacts

PART II: NRC LICENSEE AND FOREIGN POWER REACTOR DATA

SECTION 1. U.S. COMMERCIAL NUCLEAR POWER REACTORS: GENERAL DATA BY UNIT NAME

SECTION 2. U.S. COMMERCIAL NUCLEAR POWER PLANTS FORMERLY LICENSED TO OPERATE

SECTION 3. U.S. REACTOR CANCELLATIONS

Units Under Construction Permit Review

78-79

Units With Construction Permit

SECTION 4. U.S. NUCLEAR POWER REACTORS BY LICENSEE

82-83

SECTION 5. WORLD LIST OF NUCLEAR POWER REACTORS 
PART I: NRC OVERVIEW 


\section{MISSION}

TO ENSURE THAT THE CIVILIAN USES OF NUCLEAR MATERIALS AND FACILITIES ARE CONDUCTED IN A MANNER CONSISTENT WITH PUBLIC HEALTH AND SAFETY, ENVIRONMENTAL QUALITY, NATIONAL SECURITY, AND

ANTITRUST LAWS. THE MAJOR SHARE OF THE NRC's WORK IS FOCUSED ON REGULATING THE USE OF NUCLEAR ENERGY TO GENERATE ELECTRIC POWER.

\section{STATUTORY AUTHORITY}

- ATOMIC ENERGY ACT OF 1954, AS AMENDED.

- ENERGY REORGANIZATION ACT OF 1974, AS AMENDED

- URANIUM MILL TAILINGS RADIATION CONTROL ACT OF 1978.

- NUCLEAR NON-PROLIFERATION ACT OF 1978.

- loW-leVel radioActive waste policy act Of 1980.

- NUCleAR WASTE POLICY ACT OF 1982.

- LOW-LEVEL RADIOACTIVE WASTE POLICY AMENDMENTS ACT OF 1985.

- DIPLOMATIC SECURITY AND ANTI-TERRORISM ACT OF 1986.

- NUCLEAR WASTE POLICY AMENDMENTS ACT OF 1987. 


\section{MAJOR ACTIVITIES}

NRC FULFILLS ITS RESPONSIBILITIES THROUGH A SYSTEM OF LICENSE AND REGULATORY ACTIVITIES THAT INCLUDE:

- LICENSING THE CONSTRUCTION AND OPERATION OF NUCLEAR REACTORS AND OTHER NUCLEAR FACILITIES.

- LICENSING THE POSSESSION, USE, PROCESSING, HANDLING, AND DISPOSAL OF NUCLEAR MATERIAL.

- DEVELOPING AND IMPLEMENTING RULES AND REGULATIONS THAT GOVERN LICENSED NUCLEAR ACTIVITIES.

- INSPECTING LICENSED FACILITIES AND ACTIVITIES.

- INVESTIGATING NUCLEAR INCIDENTS AND ALLEGATIONS CONCERNING ANY MATTER REgULATED BY NRC.

- ENFORCING NRC LICENSES AND REGULATIONS.

- CONDUCTING PUBLIC HEARINGS ON MATTERS OF NUCLEAR AND RADIOLOGICAL SAFETY, ENVIRONMENTAL CONCERN, COMMON DEFENSE AND SECURITY, AND ANTITRUST LAWS.

- DEVELOPING EFFECTIVE WORKING RELATIONSHIPS WITH THE STATES REGARDING REGULATION OF NUCLEAR MATERIAL. 


\section{ORGANIZATION}

FIVE-MEMBER COMMISSION APPOINTED BY THE PRESIDENT WITH THE ADVICE AND CONSENT OF THE SENATE; STAGGERED, 5-YEAR TERM APPOINTMENTS.

PRINCIPAL OFFICES

- NUCLEAR REACTOR REgULATION - DIRECTS ALL LICENSE AND INSPECTION ACTIVITIES ASSOCIATED WITH THE DESIGN, CONSTRUCTION, AND OPERATION OF NUCLEAR POWER PLANTS AND NONPOWER REACTORS.

- NUCLEAR MATERIAL SAFETY AND SAFEGUARDS - DIRECTS ALL LICENSE AND INSPECTION ACTIVITIES ASSOCIATED WITH NUCLEAR FUEL CYCLE FACILITIES, USERS OF NUCLEAR MATERIAL, TRANSPORT OF NUCLEAR MATERIAL, LICENSE ACTIVITIES INVOLVING THE EXPORT OF SPECIAL NUCLEAR MATERIAL, AND DECONTAMINATION AND DECOMMISSIONING OF FACILITIES AND SITES.

- NUCLEAR REGULATORY RESEARCH - PROVIDES INDEPENDENT EXPERTISE AND INFORMATION FOR MAKING TIMELY REGULATORY JUDGEMENTS, RESOLVING SAFETY ISSUES AND DEVELOPING TECHNICAL REGULATIONS AND STANDARDS. ANTICIPATES PROBLEMS OF POTENTIAL SAFETY SIGNIFICANCE FOR WHICH NEW OR EXPANDED KNOWLEDGE CAN ASSIST THE NRC IN PURSUING ITS MISSION.

- ANALYSIS AND EVALUATION OF OPERATIONAL DATA - COLLECTS, ANALYZES, AND DISSEMINATES INFORMATION ABOUT THE OPERATIONAL SAFETY OF COMMERCIAL NUCLEAR POWER REACTORS AND CERTAIN NON-REACTOR ACTIVITIES.

- REGIONAL OFFICES - CARRY OUT INSPECTION, ENFORCEMENT, LICENSING, AND EMERGENCY RESPONSE PROGRAMS ORIGINATING IN THE HEADQUARTERS' OFFICES.

SEE ORGANIZATIONAL STRUCTURE ON PAGES 4-5. 


\section{U.S. NUCLEAR REGULATORY COMMISSION}

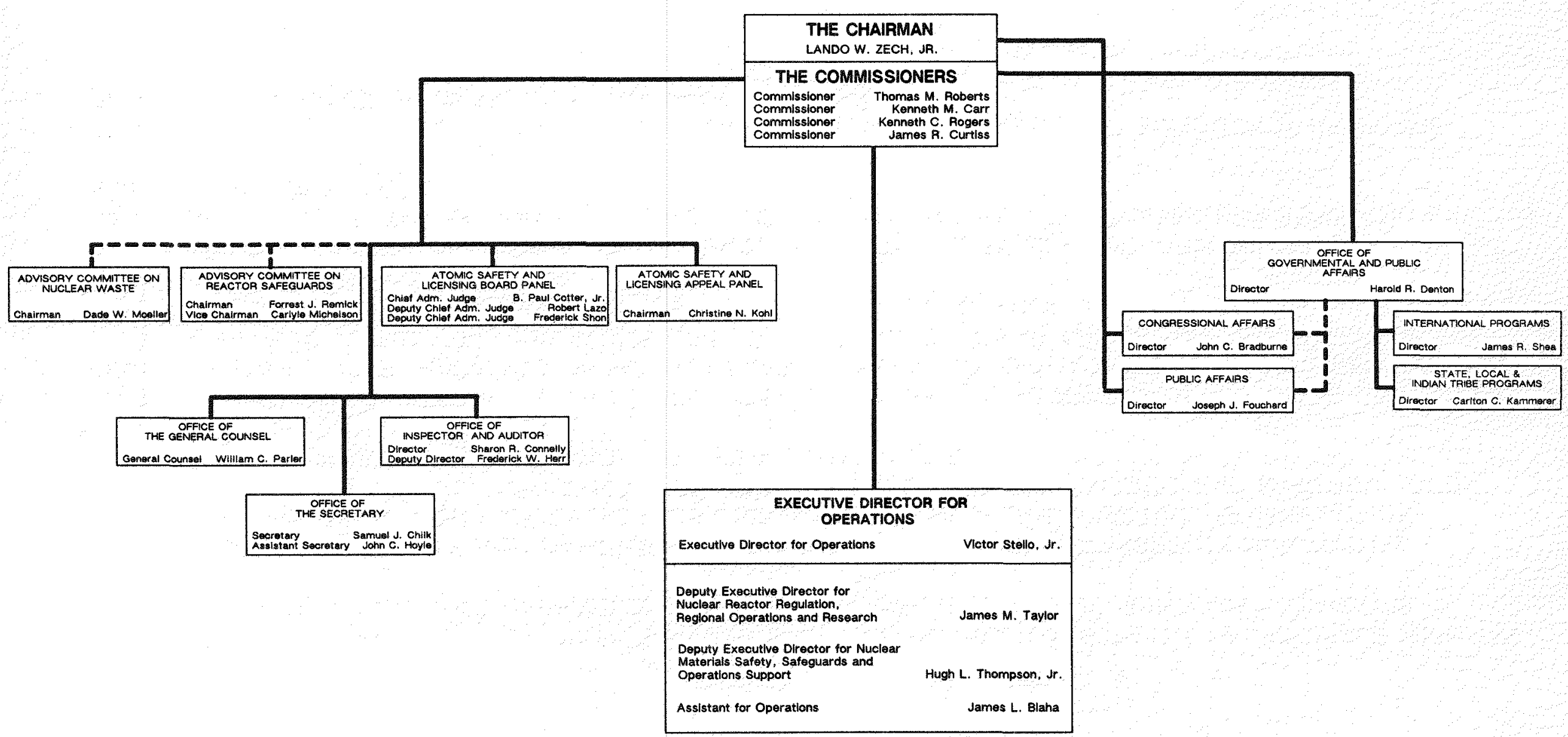




\section{U.S. NUCLEAR REGULATORY COMMISSION}

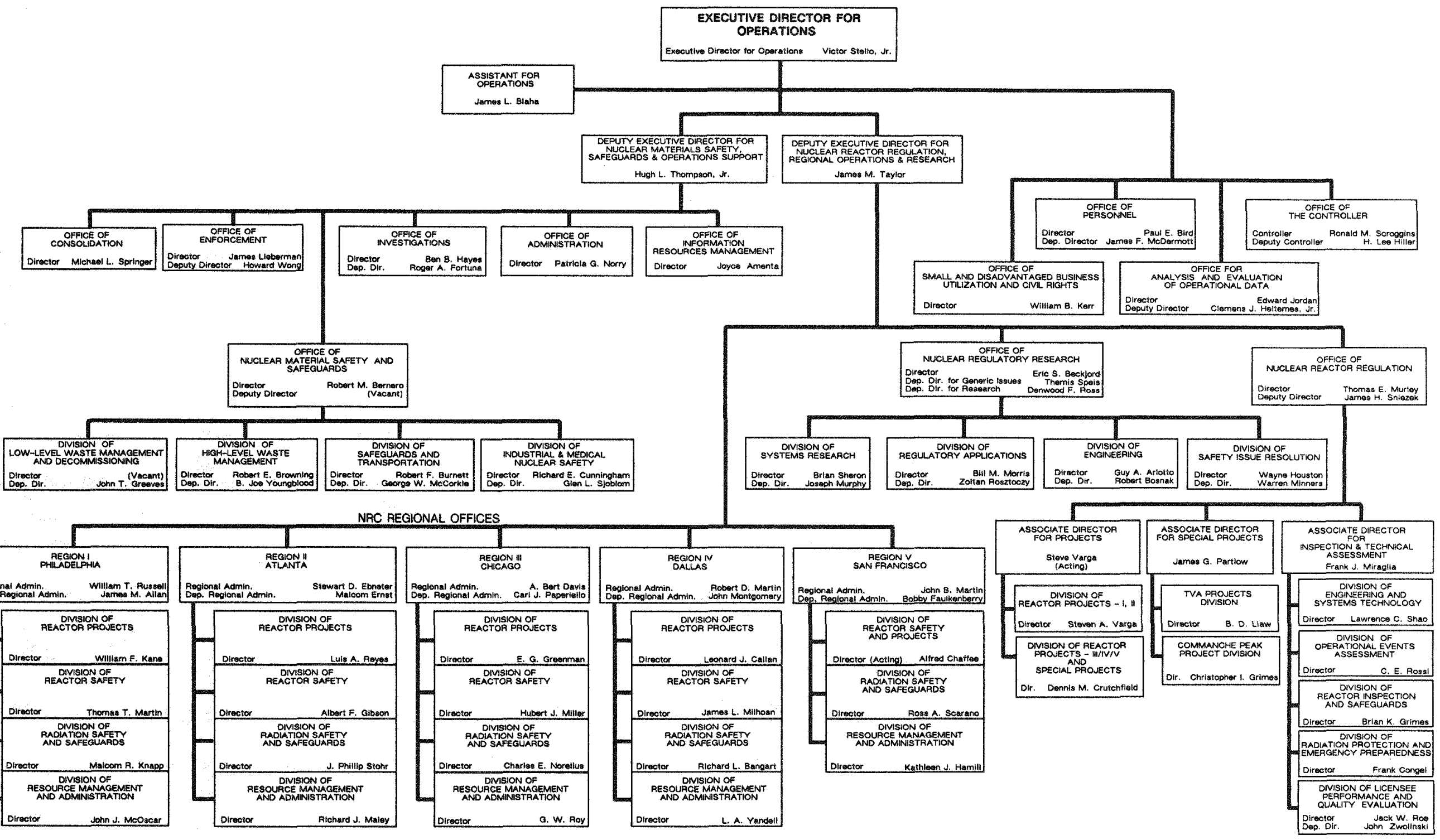




\section{GEOGRAPHIC LOCATIONS}

HEADQUARTERS

- GREATER WASHINGTON, DC AREA (7 DIFFERENT LOCATIONS).

- PROCEEDING WITH TWO-STEP CONSOLIDATION IN ROCKVILLE, MARYLAND.

- OVER ONE-HALF OF HEADQUARTERS EMPLOYEES CONSOLIDATED AT ONE WHITE FLINT NORTH BY APRIL 1988.

- GSA EXPECTED TO CONCLUDE AGREEMENT WITH DEVELOPERS BY SPRING 1989 TO CONSTRUCT ADJACENT OFFICE BUILDING TO HOUSE REMAINDER OF HEADQUARTERS STAFF.

FIVE REGIONAL OFFICES

- REGION I, KING OF PRUSSIA, PA.

- REgion II, ATLANTA, Ga.

- REGION III, GLEN ELLYN, IL.

- REgION IV, ARLINGTON, TX.

- REgION V, WALNUT CREEK, CA.

TECHNICAL TRAINING CENTER - CHATTANOOGA, TN.

URANIUM RECOVERY FIELD OFFICE - GOLDEN, CO.

RESIDENT INSPECTORS AT ALL NUCLEAR POWER PLANT SITES (SEE EXHIBIT 3.1, PAGE 8). 


\section{U.S. NUCLEAR REGULATORY COMMISSION GEOGRAPHIC LOCATIONS}

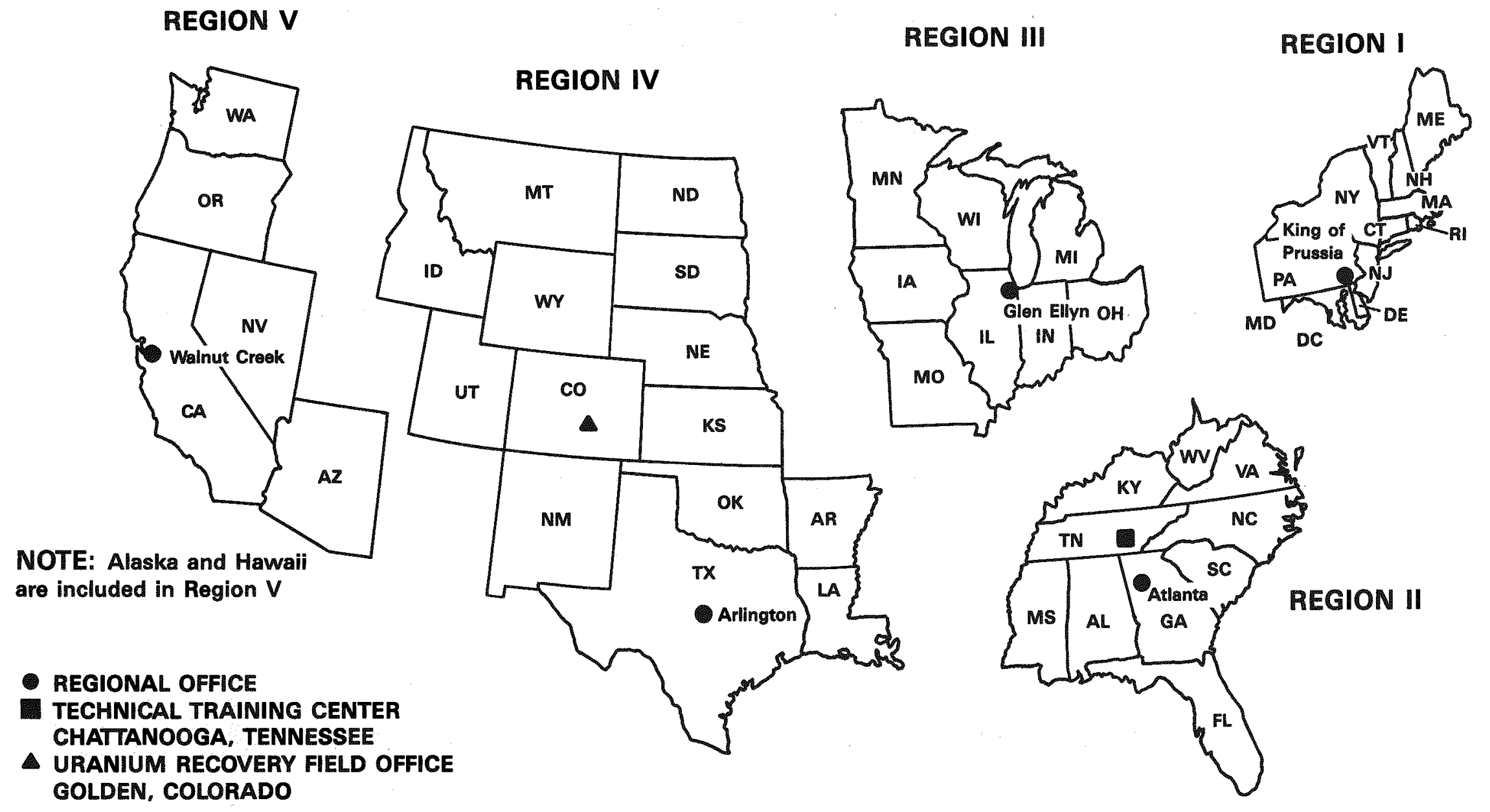




\section{RESOURCE PROFILES}

STAFFING

- FY 1989 ALlowANCE 3,180 FTE.

- ABOUT 68\% AT HEADQUARTERS, 27\% IN REgIONS, AND 5\% AT REACTOR SITES.

- Allocation by Mission AREa (SEe EXHIBIT 5, PAge 11).

FUNDING

- FY 1989 BUDGET \$420 MILLION.

- ABOUT 63\% OF NRC BUDGET REQUIRED FOR SALARIES, LOGISTICAL SUPPORT AND TRAVEL ISEE EXHIBIT 4, PAGE 10).

- allocation by mission area (SEe eXHibit 5, PAge 11).

- FY 1989 BUDGET IN 1975 (YEAR NRC EST.) CONSTANT DOLLARS EQUATES TO \$208 MILLION (SEE EXHIBIT 6, PAGE 12). THERE WERE 51 REACTORS LICENSED TO OPERATE IN OCTOBER 1975, IN DECEMBER 1988 THERE WERE 111 .

\section{LICENSE FEES}

- PUBLIC LAW 100-203 AUTHORIZED NRC TO COllect FEes that TOTAL AT LEAST 45\% OF ITS ANNUAL BUDGET (FY 1988-1989 ONLY).

- NRC's AUTHORITY TO COLLECT FEES WILL REVERT TO THE PERCENT REQUIREMENTS ESTABLISHED BY PUBLIC LAW 99-272 AND WILL BE CAPPED AT NOT MORE THAN 33\% OF ITS ANNUAL BUDGET AFTER FY 1989. 


\section{NRC FY 1989 STAFF RESOURCES BY LOCATION}

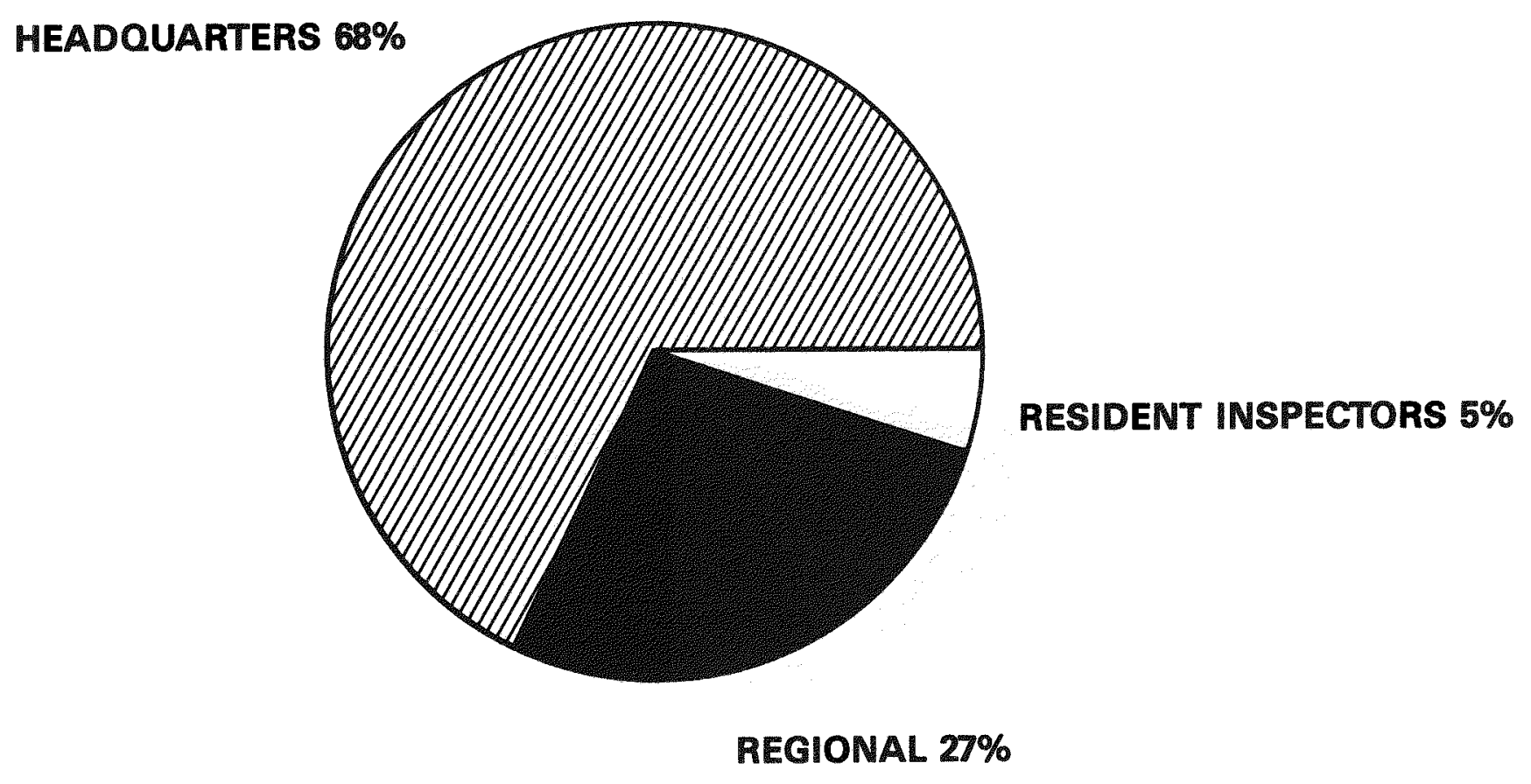




\section{NRC FY 1989 STAFF RESOURCES BY FUNCTION}
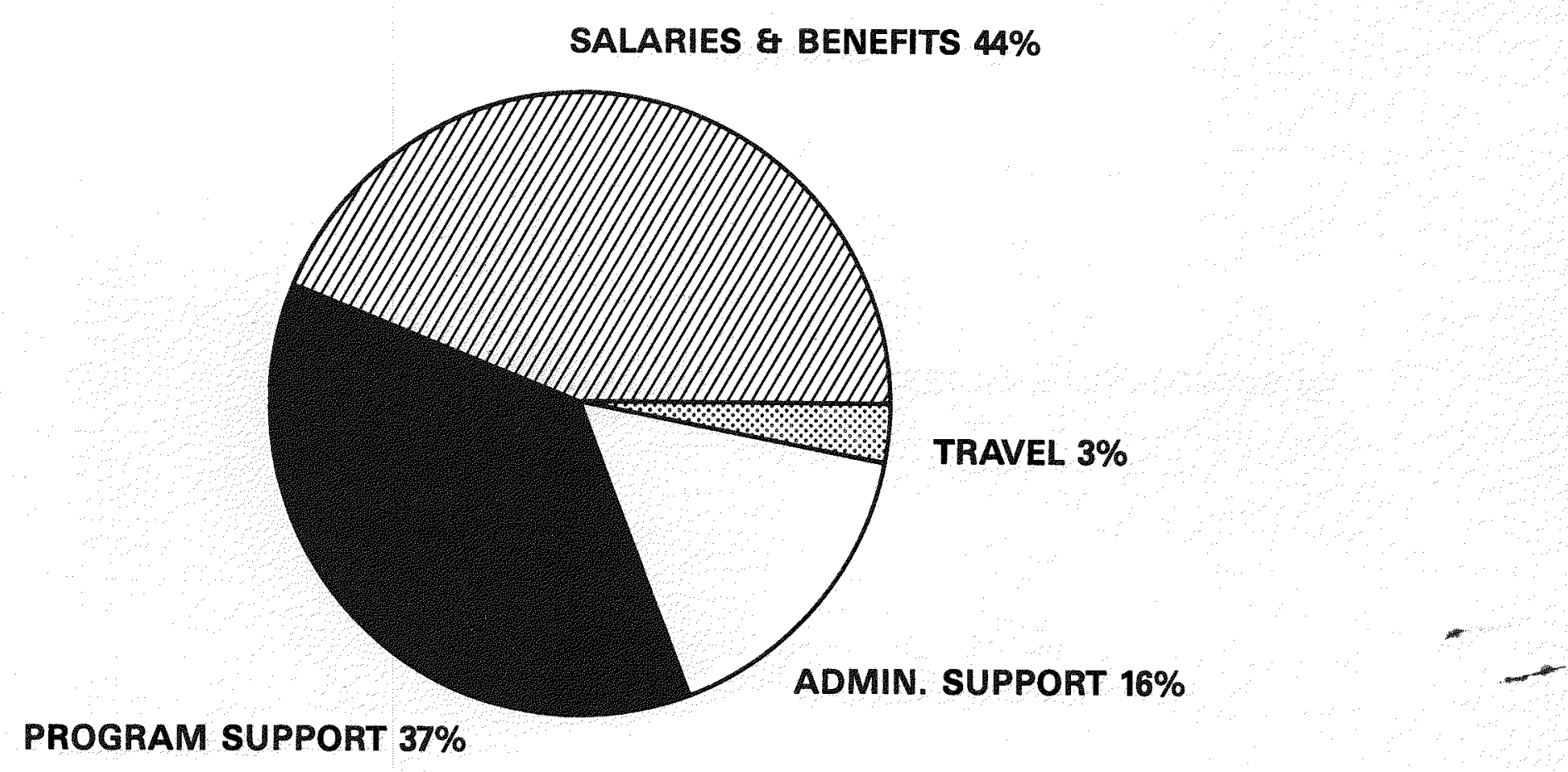


\section{NRC FY 1989 RESOURCES BY MISSION AREA}

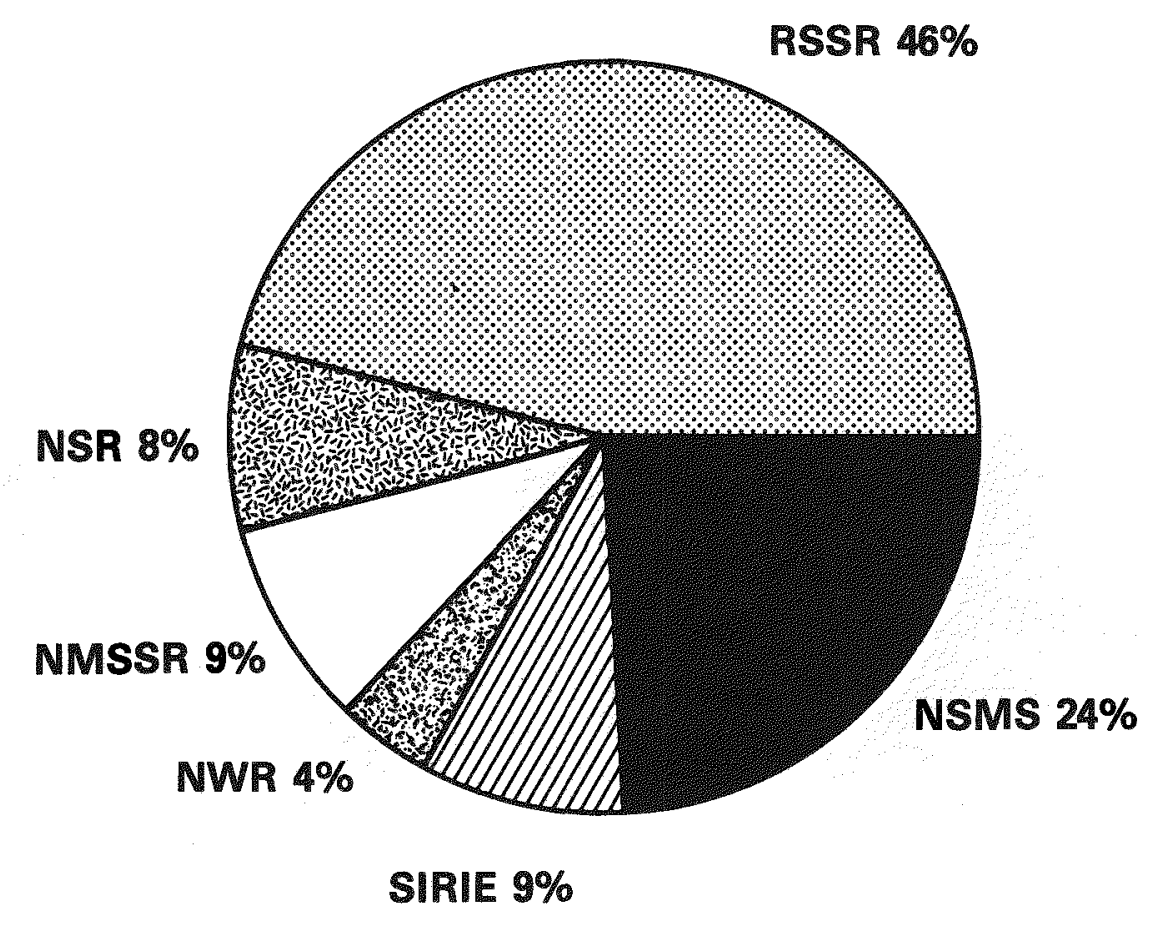

STAFF

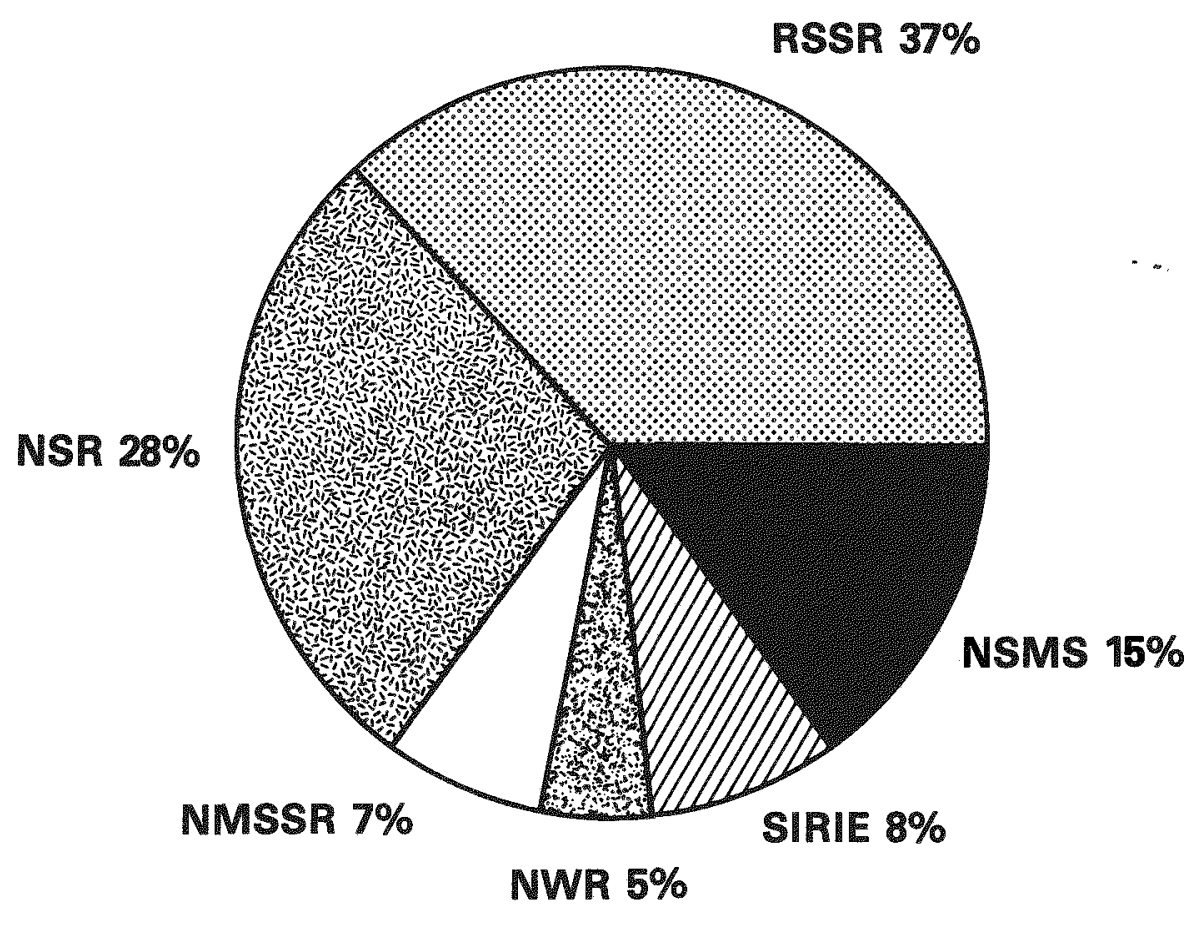

DOLLARS

\section{LEGEND}

REACTOR SAFETY \& SAFEGUARDS REGULATION (RSSR)

NUCLEAR SAFETY RESEARCH (NSR)

NUCLEAR MATERIAL SAFETY \& SAFEGUARDS REGULATION (NMSSR)

NUCLEAR WASTE REGULATION (NWR)

SPECIAL/INDEPENDENT REVIEWS, INVESTIGATIONS \& ENFORCEMENT (SIRIE)

NUCLEAR SAFETY MANAGEMENT \& SUPPORT (NSMS) 


\section{U.S. NUCLEAR REGULATORY COMMISSION TOTAL OBLIGATIONS IN CONSTANT DOLLARS (DOLLARS IN MILLIONS)}

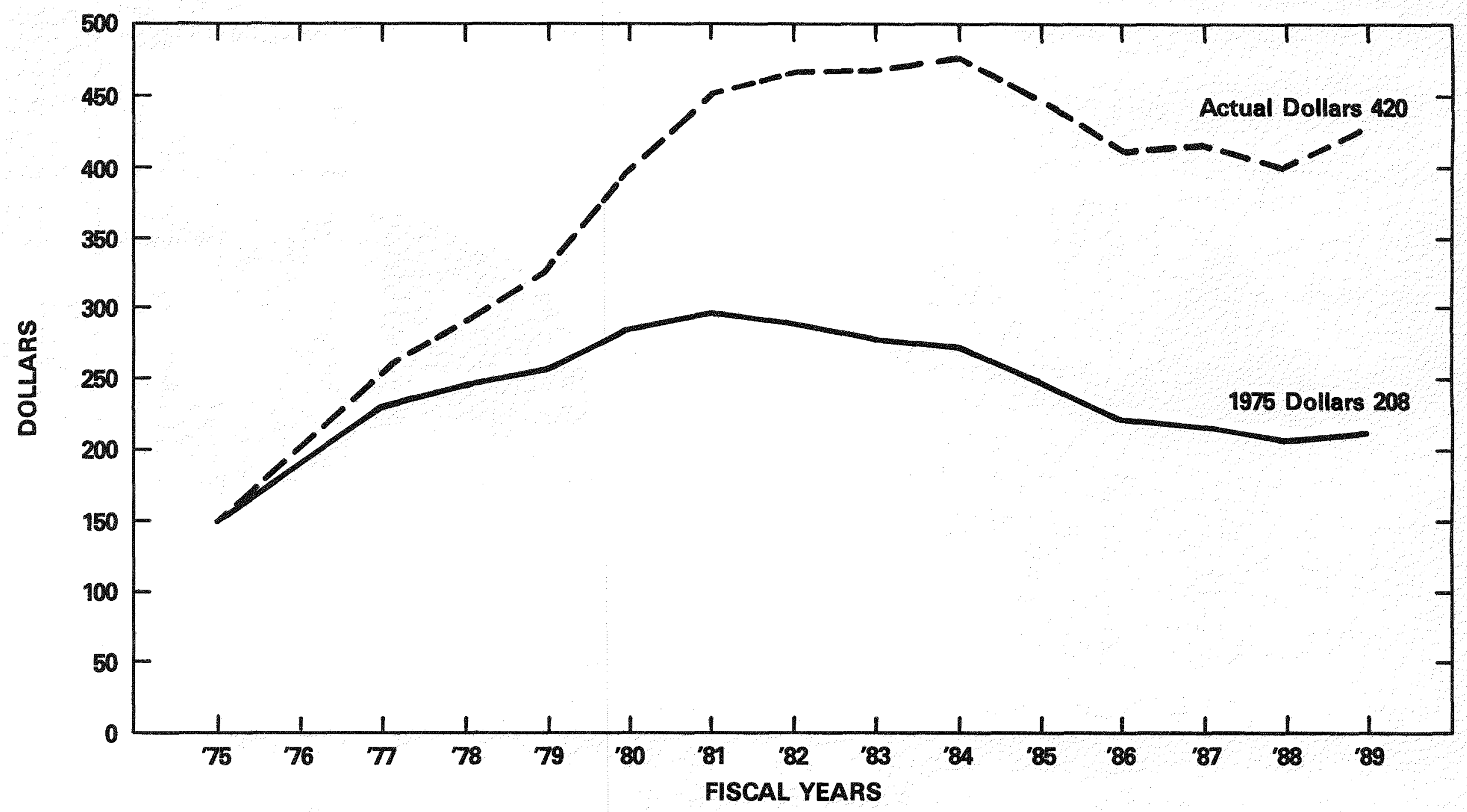




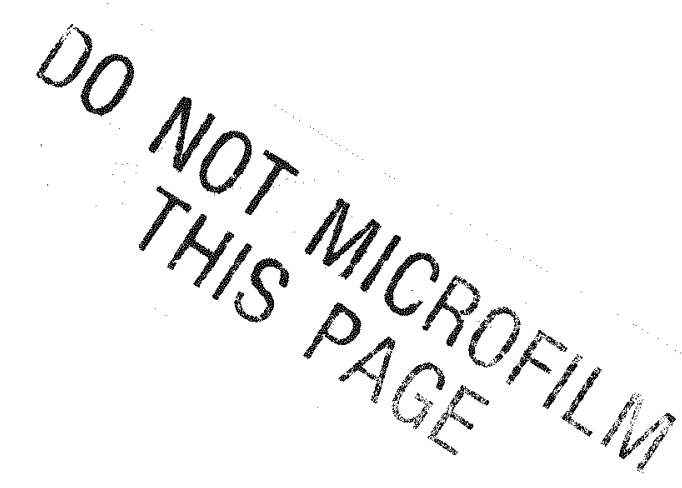

Page 13 


\section{NUCLEAR POWER REACTORS}

OPERATING AND UNDER CONSTRUCTION (SEE EXHIBITS 7.1-7.6 PAGES 15-20)

- 111 LICENSED TO OPERATE.

- 5 SCHEDULED FOR COMPLETION BY 1995.

- 7 PARTIALLY COMPLETED BUT CONSTRUCTION DEFERRED.

REACTOR DIVERSITY

- ALTHOUgh theRe ARE MANY SIMILARITIES, EACH REACTOR DESIGN CAN BE CONSIDERED UNIQUe

- 4 REACTOR VENDORS.

- 54 LICENSEES.

- 81 DIFFERENT DESIGNS.

- 75 SITES.

- 34 STATES.

ABOUT 5,400 LICENSED RACTOR OPERATORS

- EACH OPERATOR IS LICENSED For A SPECIFIC REACTOR.

- EACH OPERATOR IS REQUALIFIED PRIOR TO RENEWAL OF A SIX-YEAR LICENSE.

NRC CONDUCTS THE PRINCIPAL U.S. GOVERNMENT PROGRAM ON LIGHT WATER REACTOR SAFETY RESEARCH.

NRC CONDUCTS AROUND 3,000 POWER REACTOR INSPECTIONS ANNUALLY.

ABOUT 20 SEPARATE LICENSE CHANGES ARE REQUESTED PER POWER REACTOR EACH YEAR-RESULTS IN MORE THAN 2,000 SEPARATE NRC REVIEWS PER YEAR.

ABOUT 5,000 REACTOR EVENT REPORTS ASSESSED BY NRC ANNUALLY. 


\section{U.S. COMMERCIAL NUCLEAR POWER REACTOR SITES}

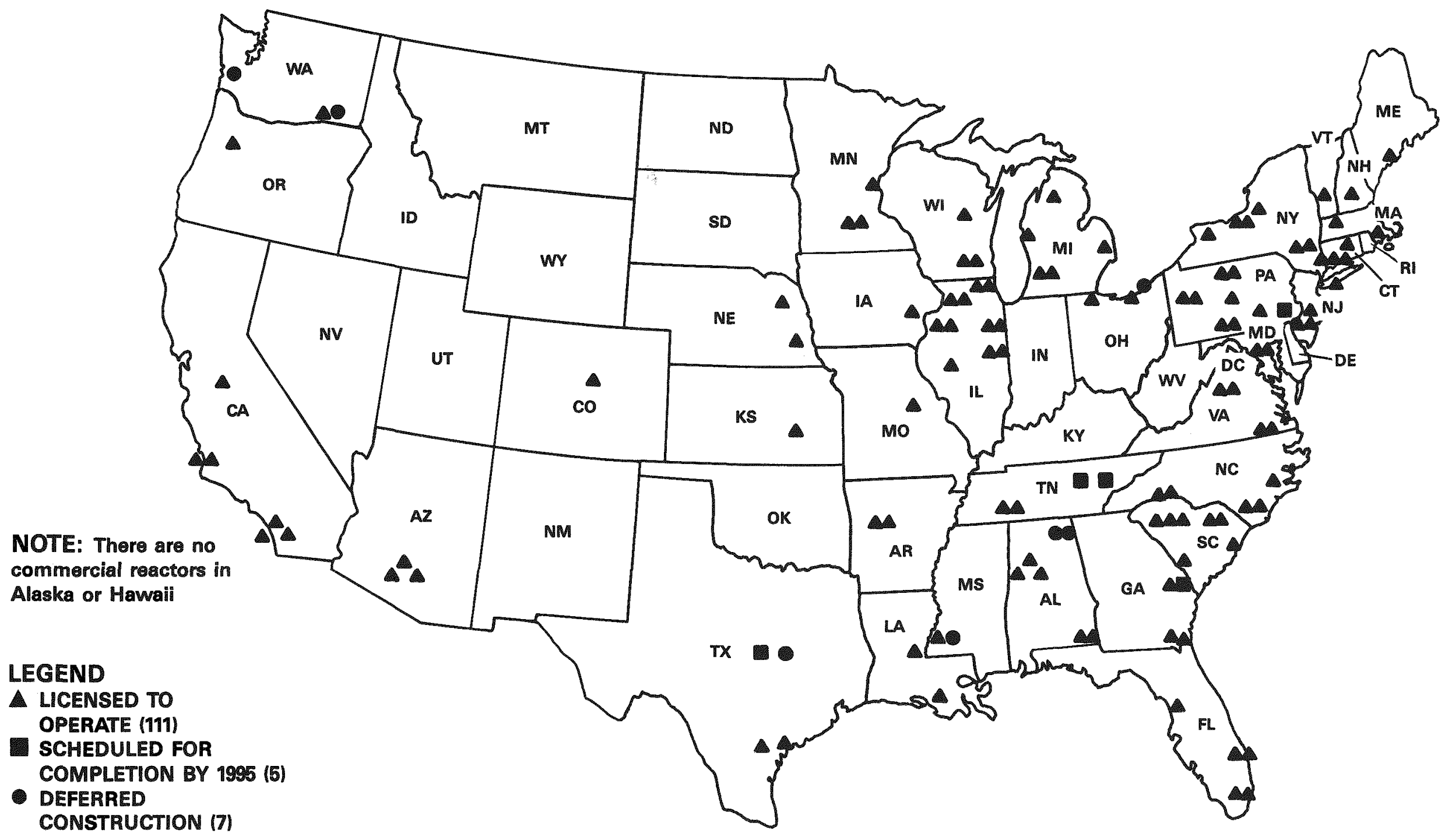




\section{NRC REGION I COMMERCIAL NUCLEAR POWER REACTOR SITES}

\section{LEGEND}

- LICENSED TO OPERATE (30) SCHEDULED FOR COMPLETION (1)

SOURCE: NRC 1/31/89

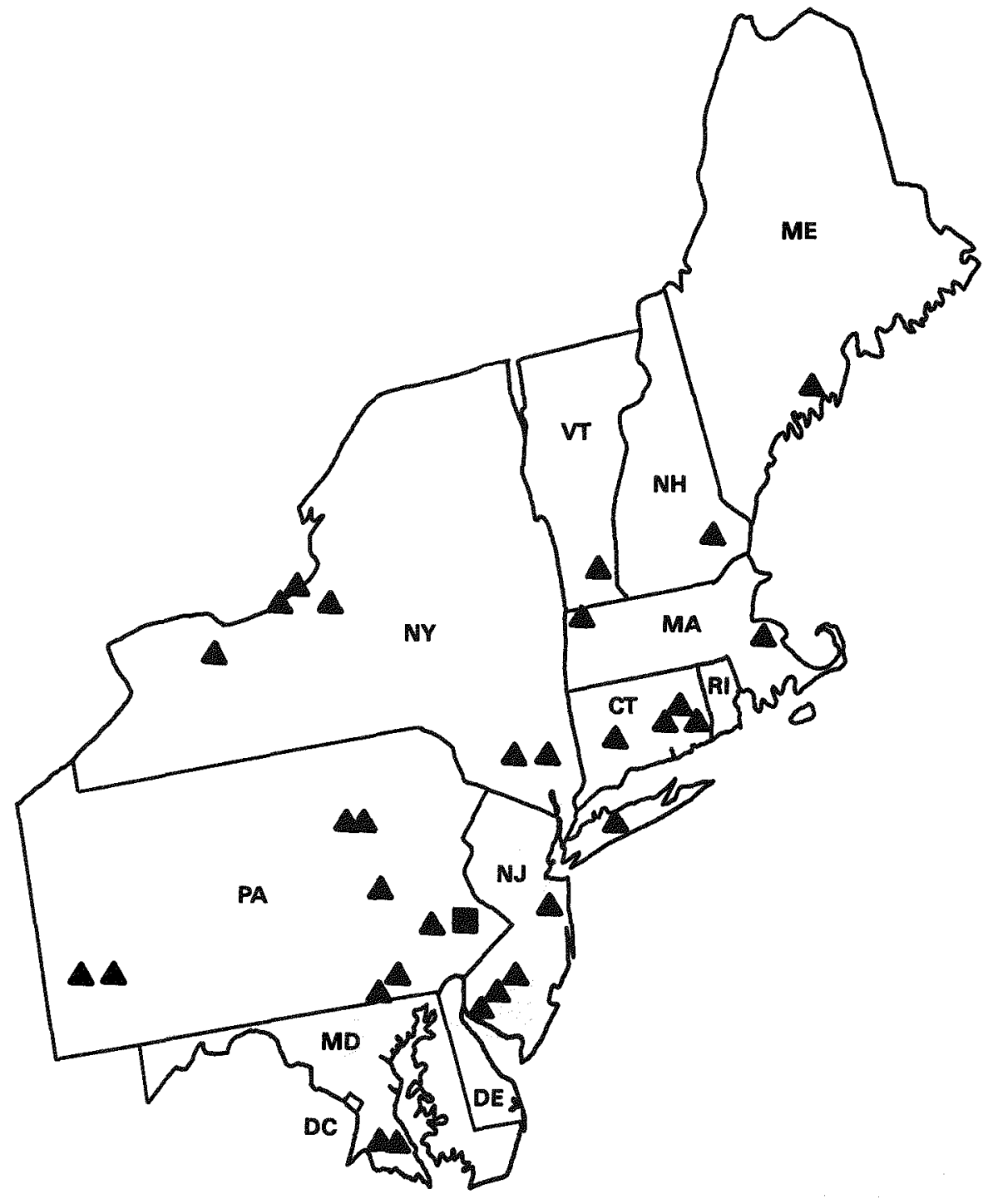

Exhibit 7.2
CONNECTICUT

HADDAM NECK

$\triangle$ MILLSTONE 1, 2, and 3 MAINE

MAINE YANKEE

MARYLAND

$\triangle$ CALVERT CLIFFS 1 and 2 MASSACHUSETTS

$\triangle$ PILGRIM 1

$\triangle$ YANKEE ROWE

NEW HAMPSHIRE

$\triangle$ SEABROOK 1 (Zero Power) NEW JERSEY

$\triangle$ HOPE CREEK 1

$\triangle$ OYSTER CREEK 1

$\triangle$ SALEM 1 and 2

NEW YORK

AITZPATRICK

A GINNA

A INDIAN POINT 2 and 3

$\Delta$ NINE MILE POINT 1 and 2

A SHOREHAM (Low Power License) PENNSYLVANIA

A BEAVER VALLEY 1 and 2

- LIMERICK 1

LIMERICK 2

$\triangle$ PEACH BOTTOM 2 and 3

4 SUSQUEHANNA 1 and 2

$\triangle$ THREE MILE ISLAND 1 VERMONT

$\triangle$ VERMONT YANKEE 


\section{NRC REGION II \\ COMMERCIAL NUCLEAR POWER REACTOR SITES}

\section{LEGEND}

LICENSED TO OPERATE (32)

SCHEDULED FOR COMPLETION (3)

- DEFERRED CONSTRUCTION (3)

SOURCE: NRC 1/31/89

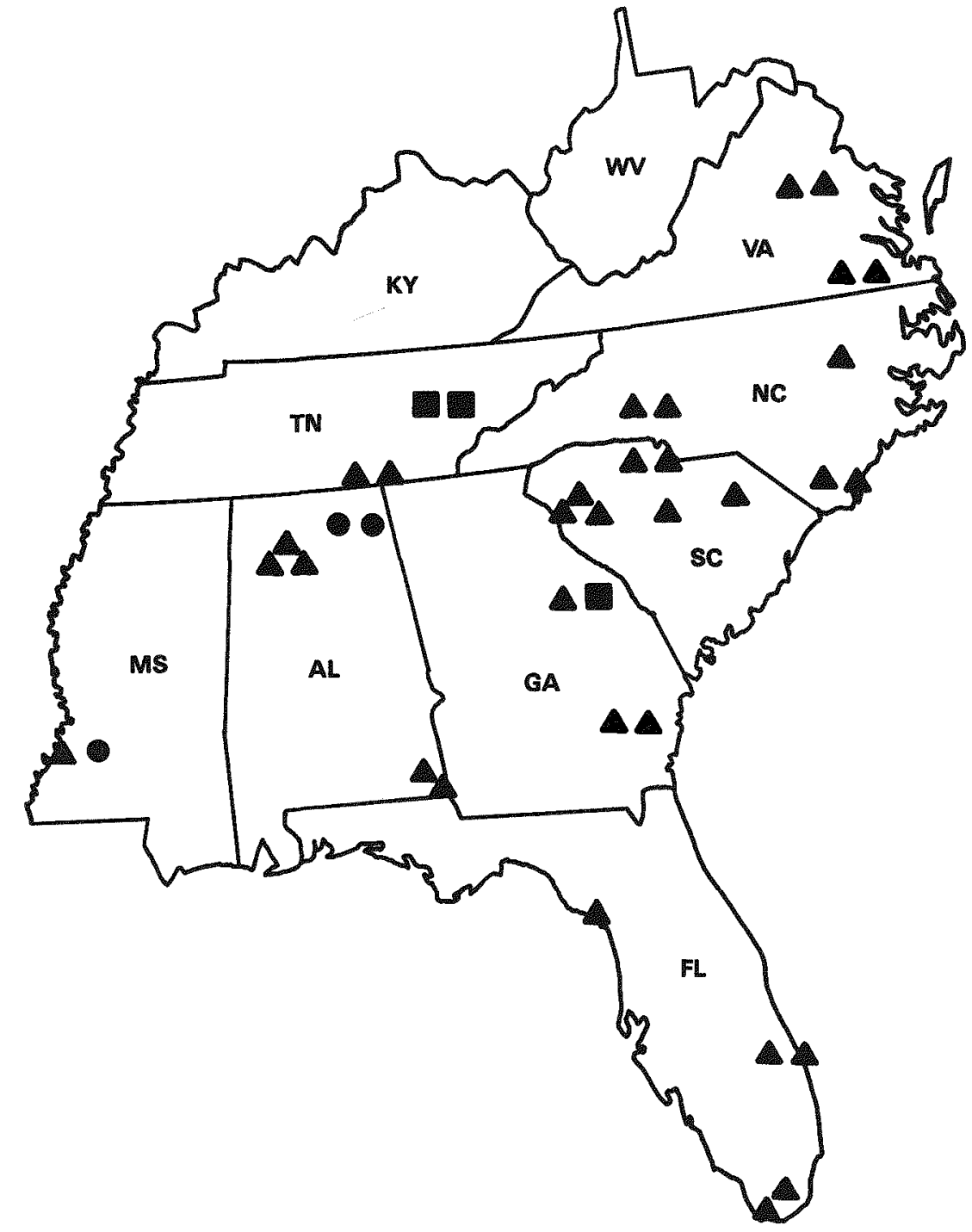

ALABAMA

$\triangle$ BROWNS FERRY 1,2 , and 3

$\triangle$ JOSEPH M. FARLEY 1 and 2

- BELLEFONTE 1 and 2 FLORIDA

A CRYSTAL RIVER 3

$\triangle$ ST. LUCIE 1 and 2

TURKEY POINT 3 and 4 GEORGIA

$\triangle$ HATCH 1 and 2

A VOGTLE 1

VOGTLE 2

MISSISSIPPI

$\triangle$ GRAND GULF 1

- GRAND GULF 2 NORTH CAROLINA

A BRUNSWICK 1 and 2

4 McGUIRE 1 and 2 A SHEARON HARRIS 1 SOUTH CAROLINA

A CATAWBA 1 and 2

$\triangle$ OCONEE 1, 2, and 3

$\triangle$ ROBINSON 2

SUMMER 1

TENNESSEE

$\triangle$ SEQUOYAH 1 and 2

WATTS BAR 1 and 2 VIRGINIA

4 NORTH ANNA 1 and 2

$\triangle$ SURRY 1 and 2 


\section{NRC REGION III \\ COMMERCIAL NUCLEAR POWER REACTOR SITES}

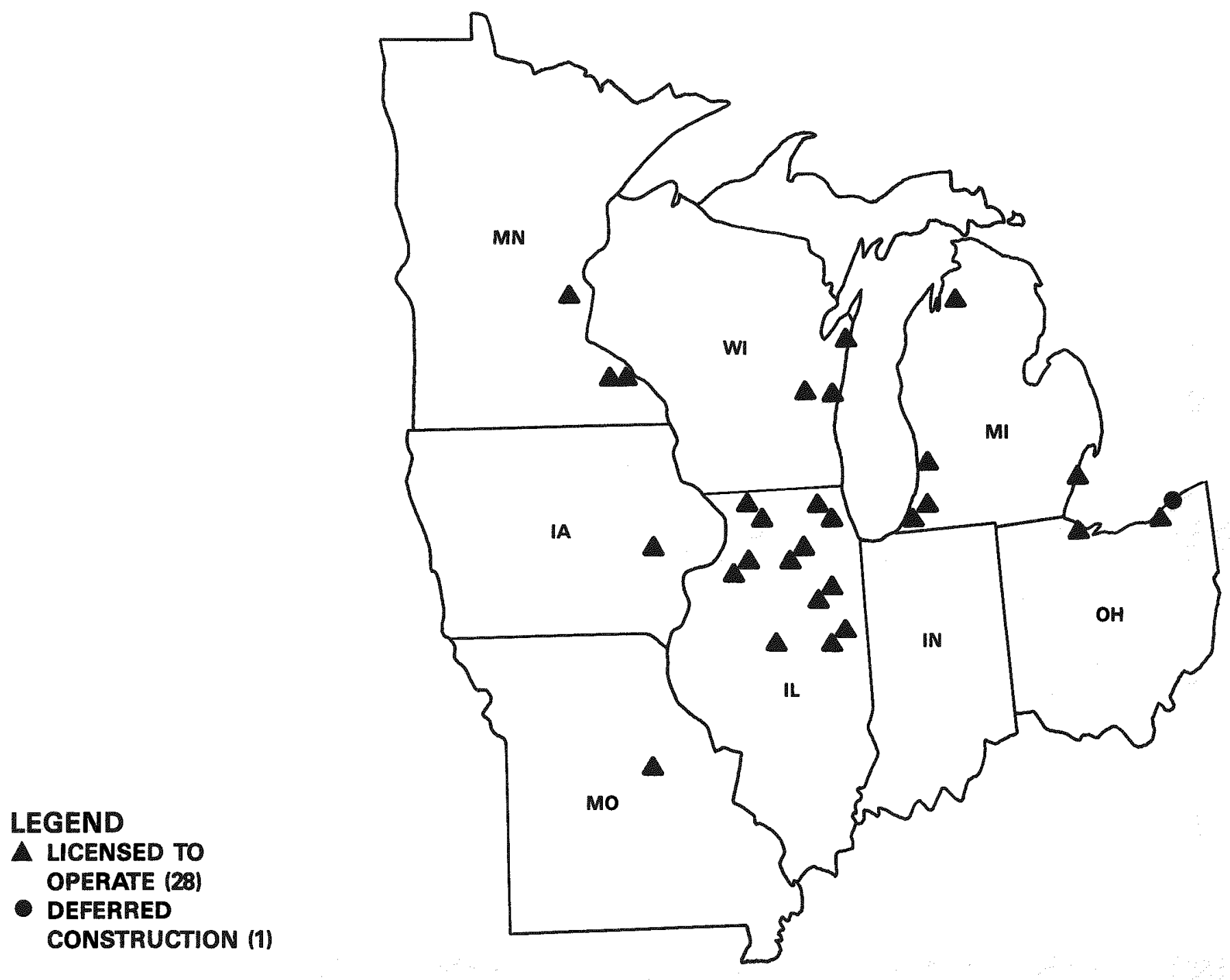

ILLINOIS

$\triangle$ BRAIDWOOD 1 and 2

BYRON 1 and 2

A CLINTON 1

1 DRESDEN 2 and 3

A LASALLE 1 and 2

$\triangle$ QUAD CITIES 1 and 2

$\triangle$ ZION 1 and 2 IOWA

$\triangle$ DUANE ARNOLD 1 MICHIGAN

A BIG ROCK POINT

COOK 1 and 2

$\triangle$ FERMI 2

$\triangle$ PALISADES

MINNESOTTA

$\triangle$ MONTICELLO

ARAIRIE ISLAND 1 and 2

MISSOURI

A CALLAWAY 1

OHIO

DAVIS-BESSE 1

$\triangle$ PERRY 1

- PERRY 2

WISCONSIN

A KEWAUNEE

4 POINT BEACH 1 and 2 


\section{NRC REGION IV COMMERCIAL NUCLEAR POWER REACTOR SITES}

\section{LEGEND}

- LICENSED TO OPERATE (10) SCHEDULED FOR COMPLETION (1)

- DEFERRED CONSTRUCTION (1)

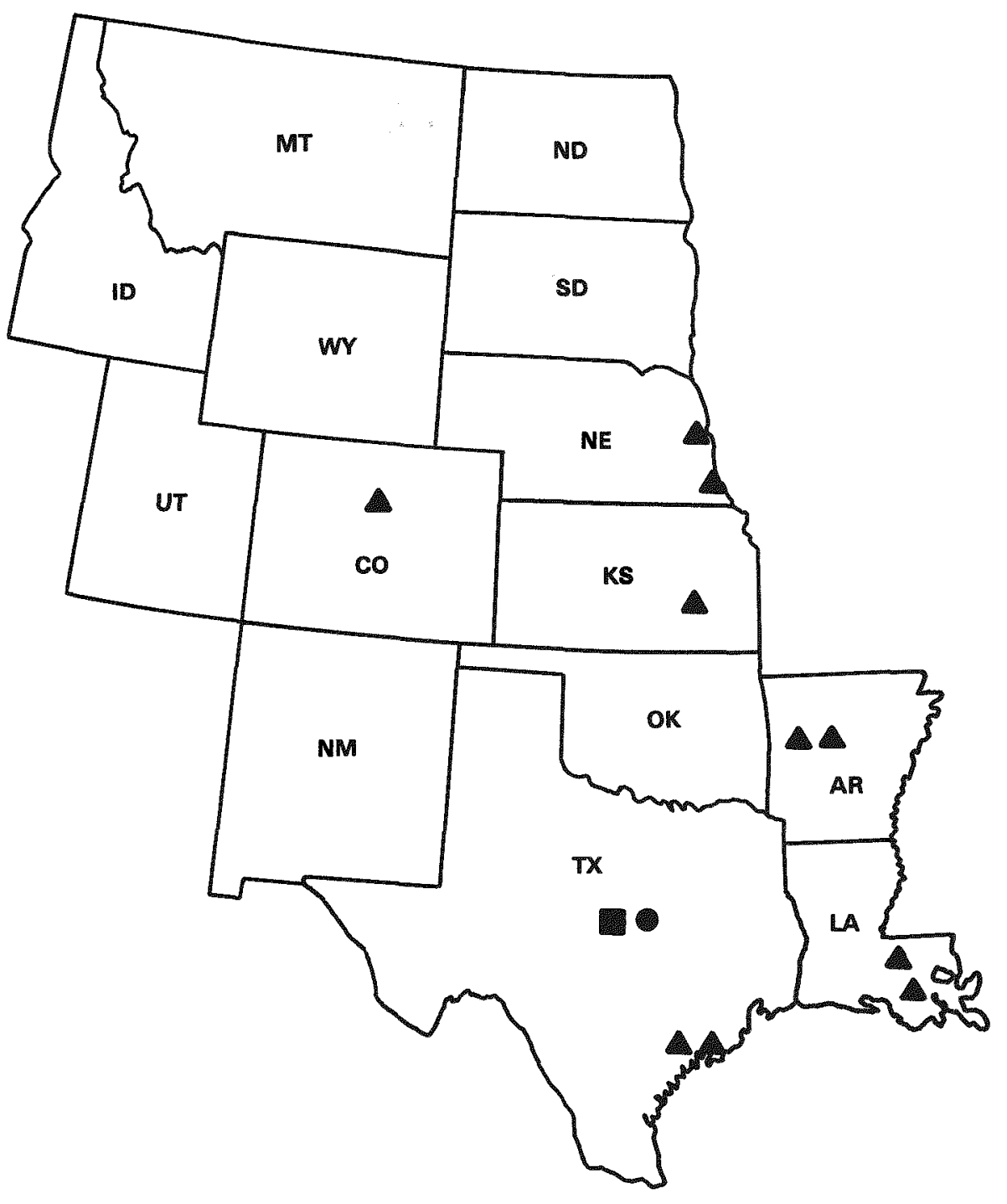

ARKANSAS

ARKANSAS 1 and 2 COLORADO

$\triangle$ FORT ST. VRAIN

KANSAS

WOLF CREEK

LOUISIANA

4 RIVER BEND 1

$\triangle$ WATERFORD 3 NEBRASKA

$\triangle$ COOPER STATION $\triangle$ FORT CALHOUN 1 TEXAS

COMANCHE PEAK 1

- COMANCHE PEAK 2

$\triangle$ SOUTH TEXAS 1

A SOUTH TEXAS 2 (Low Power License) 


\section{NRC REGION V COMMERCIAL NUCLEAR POWER REACTOR SITES}

NOTE: There are no commercial reactors in Alaska or Hawail

\section{LEGEND}

$\triangle$ LICENSED TO

OPERATE (11)

- DEFERRED

CONSTRUCTION (2)

SOURCE: NRC 1/31/89

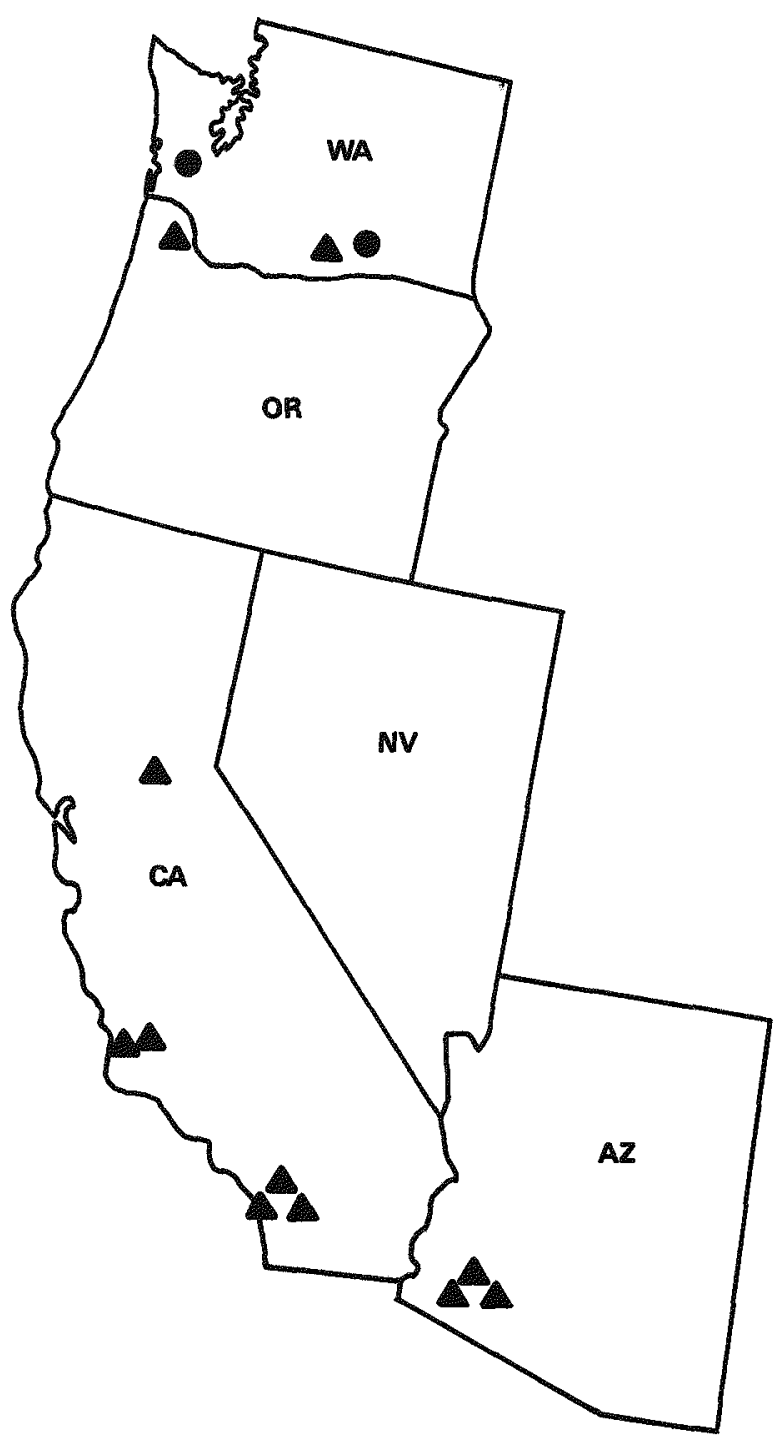

ARIZONA

$\triangle$ PALO VERDE 1,2 , and 3 CALIFORNIA

$\triangle$ DIABLO CANYON 1 and 2 $\triangle$ RANCHO SECO 1

$\triangle$ SAN ONOFRE 1, 2, and 3 OREGON

A TROJAN 1 WASHINGTON

A WPPSS 2

- WPPSS 1

WPPSS 3 


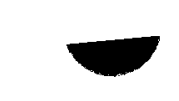

\section{DO NOT MICROFILM THIS PAGE}




\section{NUCLEAR POWER REACTORS}

111 LICENSED TO OPERATE

- GENERATE ABOUT 18\% OF NATION'S ELECTRICITY (SEE EXHIBITS 8.1-8.2, PAGES 23-24).

- REPLACEMENT ENERGY COSTS WOULD EXCEED \$15 BILLION ANNUALLY.

- AVERAGE REACTOR CAPACITY FACTOR IN 1988 ABOUT 65\% (SEE EXHIBIT 8.3, PAGE 25).

- ABOUT 28\% OF WORLD'S NUCLEAR ELECTRICITY PRODUCED IN THE U.S. (SEE EXHIBITS 9.1-9.3, PAGES 26-28). 


\section{U.S. ELECTRIC NET GENERATION BY ENERGY SOURCE}

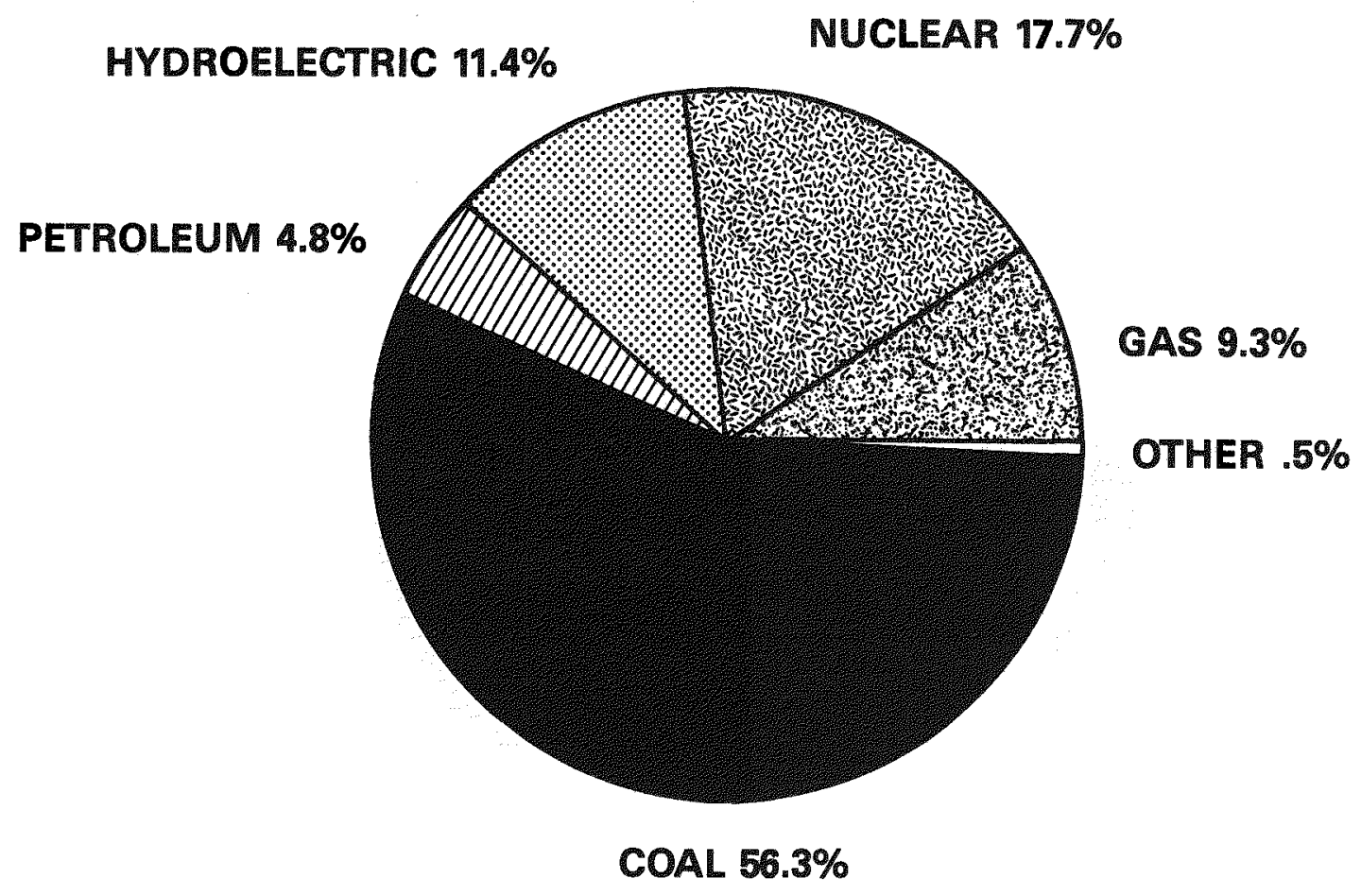




\section{U.S. ELECTRIC NET GENERATION BY NUCLEAR REACTORS IN COMMERCIAL OPERATION}

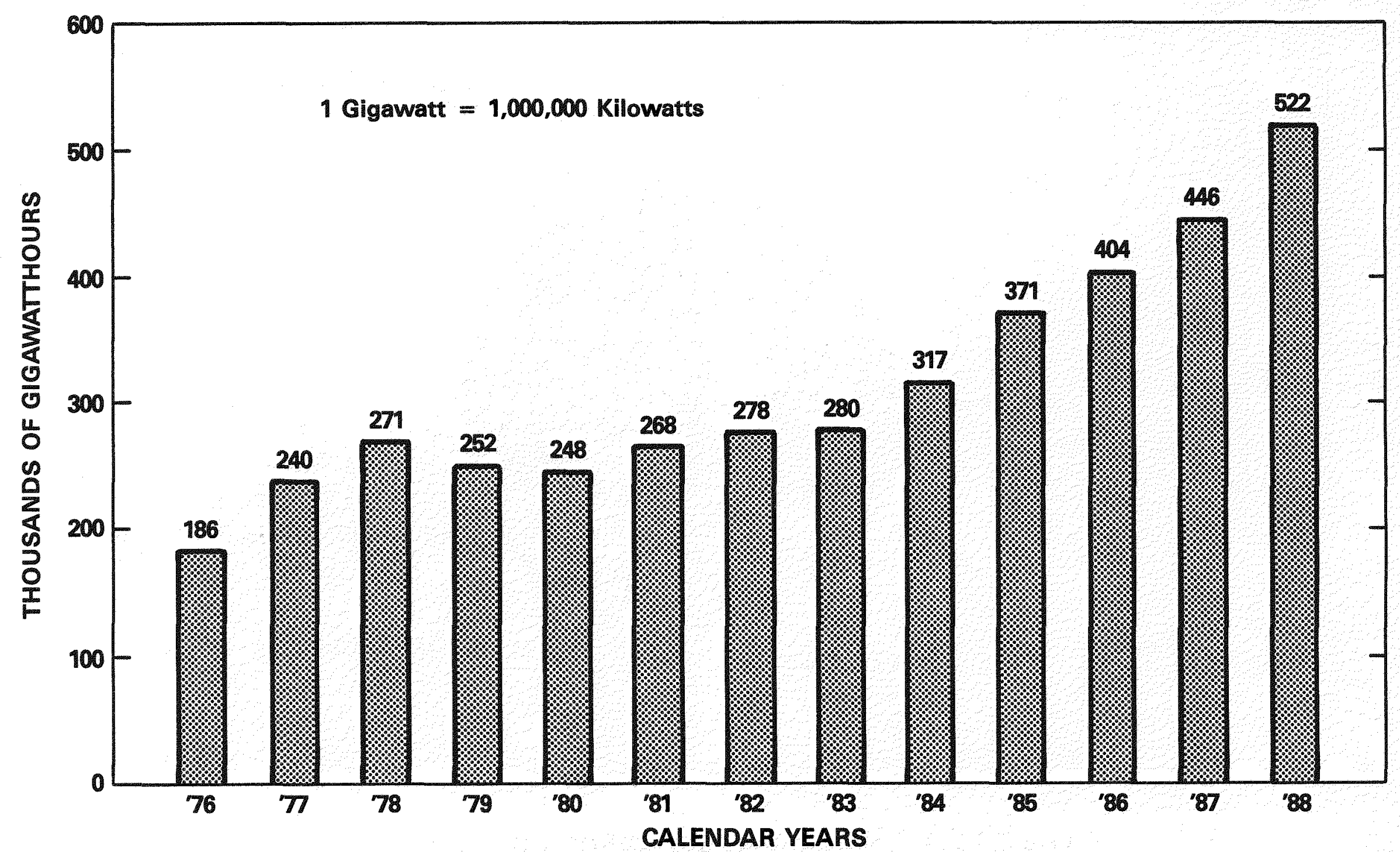




\section{AVERAGE ANNUAL U.S. REACTOR CAPACITY FACTOR USING MAXIMUM DEPENDABLE CAPACITY}

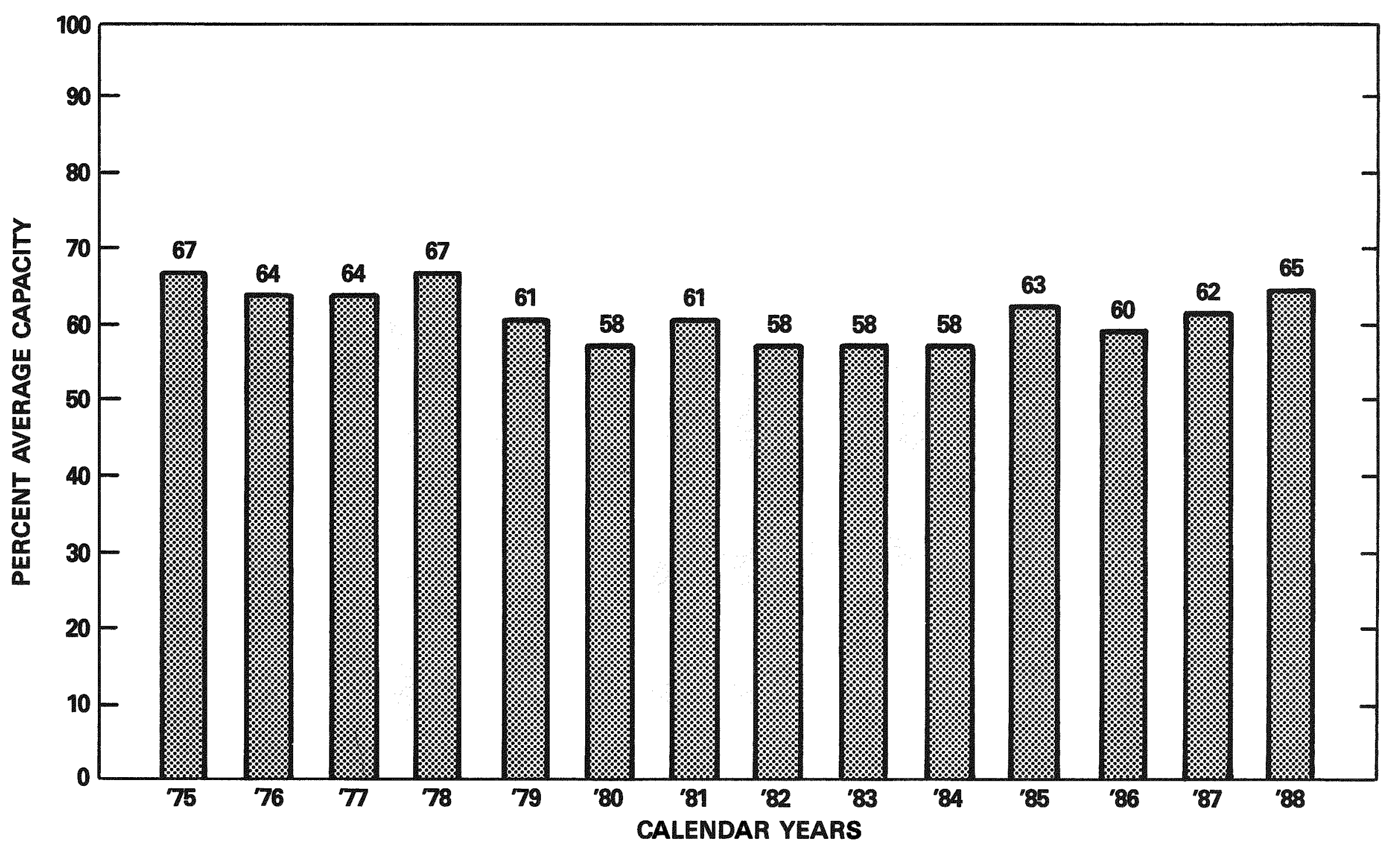




\section{WORLD ENERGY PRODUCTION BY POWER SOURCE}

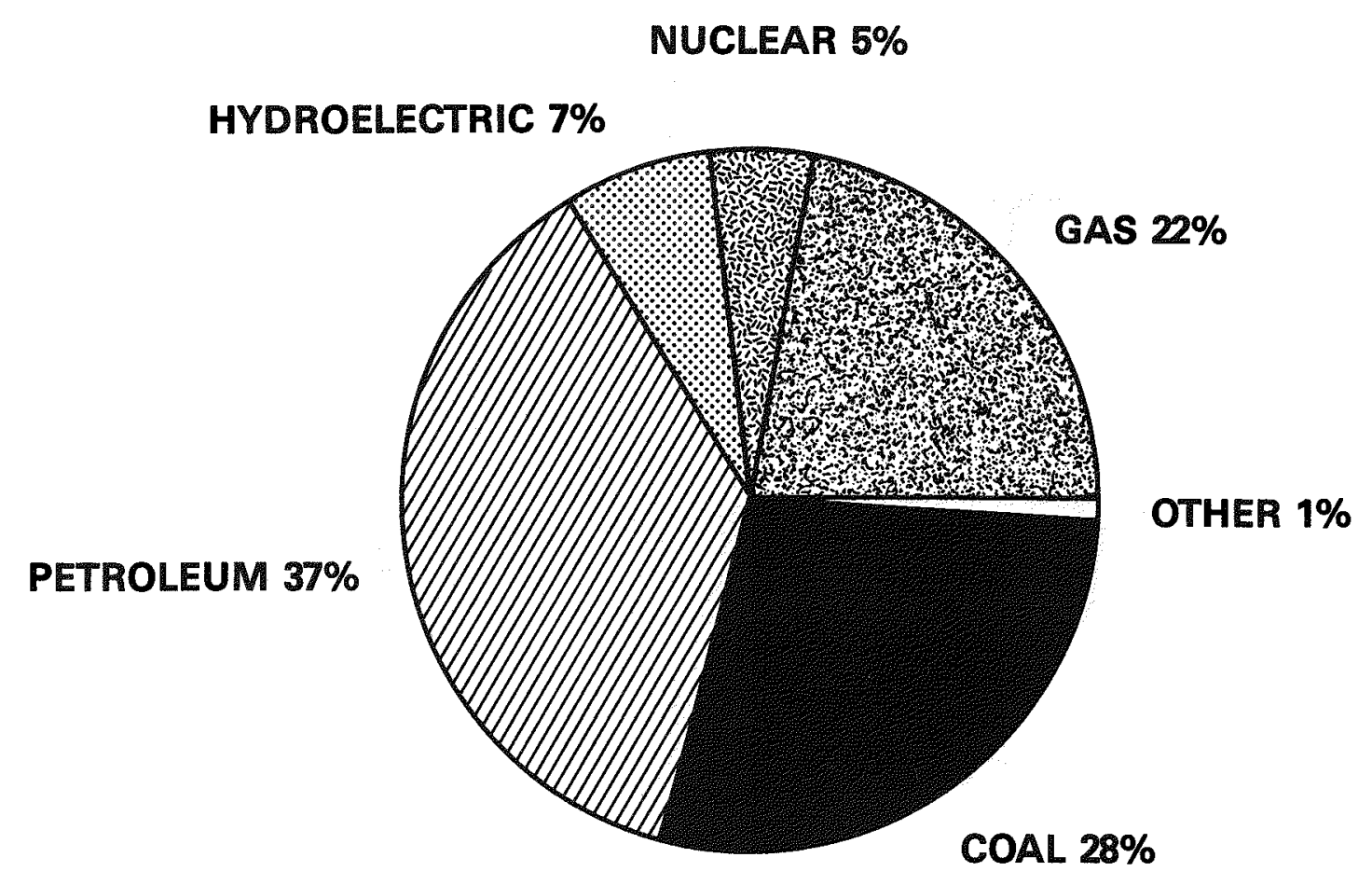




\section{WORLD NUCLEAR ELECTRIC POWER PRODUCTION BY REACTORS IN COMMERCIAL OPERATION}

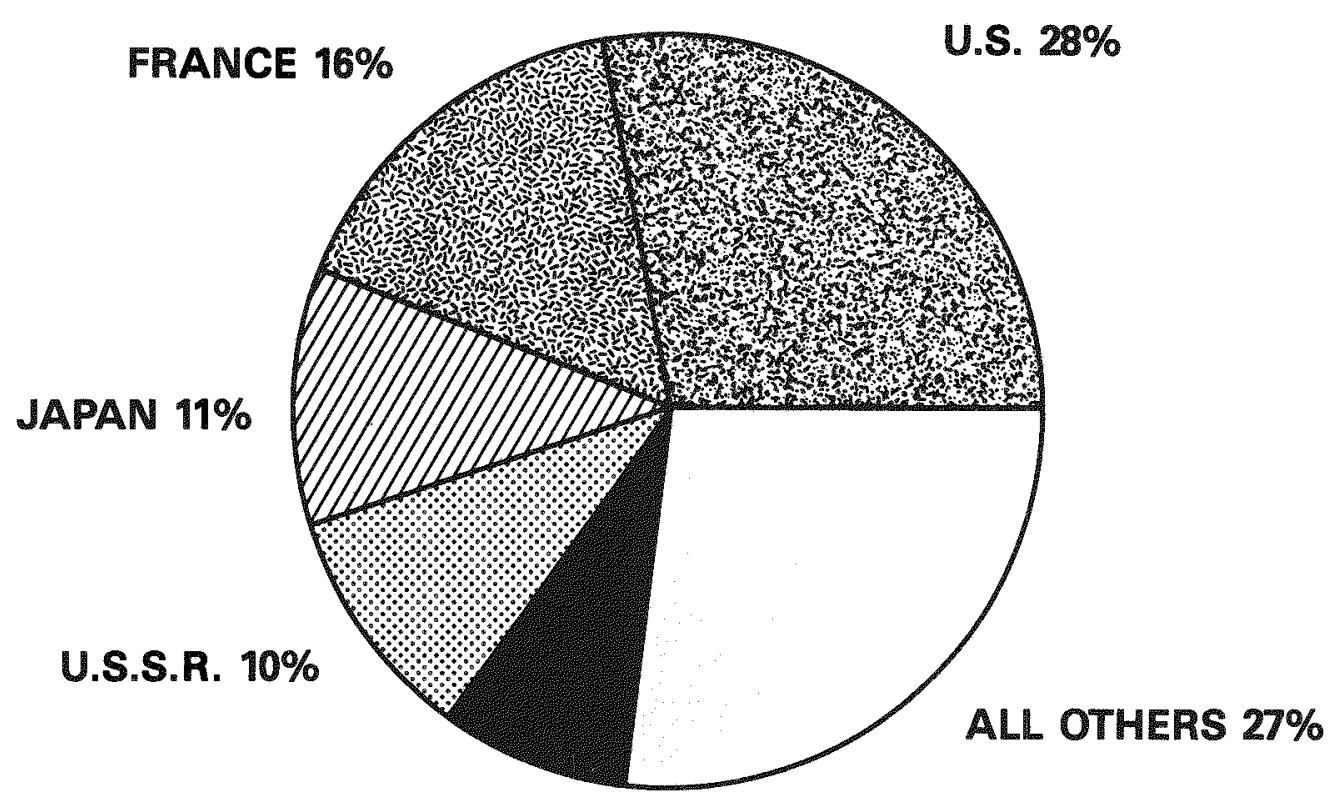

WEST GERMANY $8 \%$ 
1987 NUCLEAR ELECTRIC POWER AS PERCENT OF TOTAL ELECTRICITY PRODUCTION

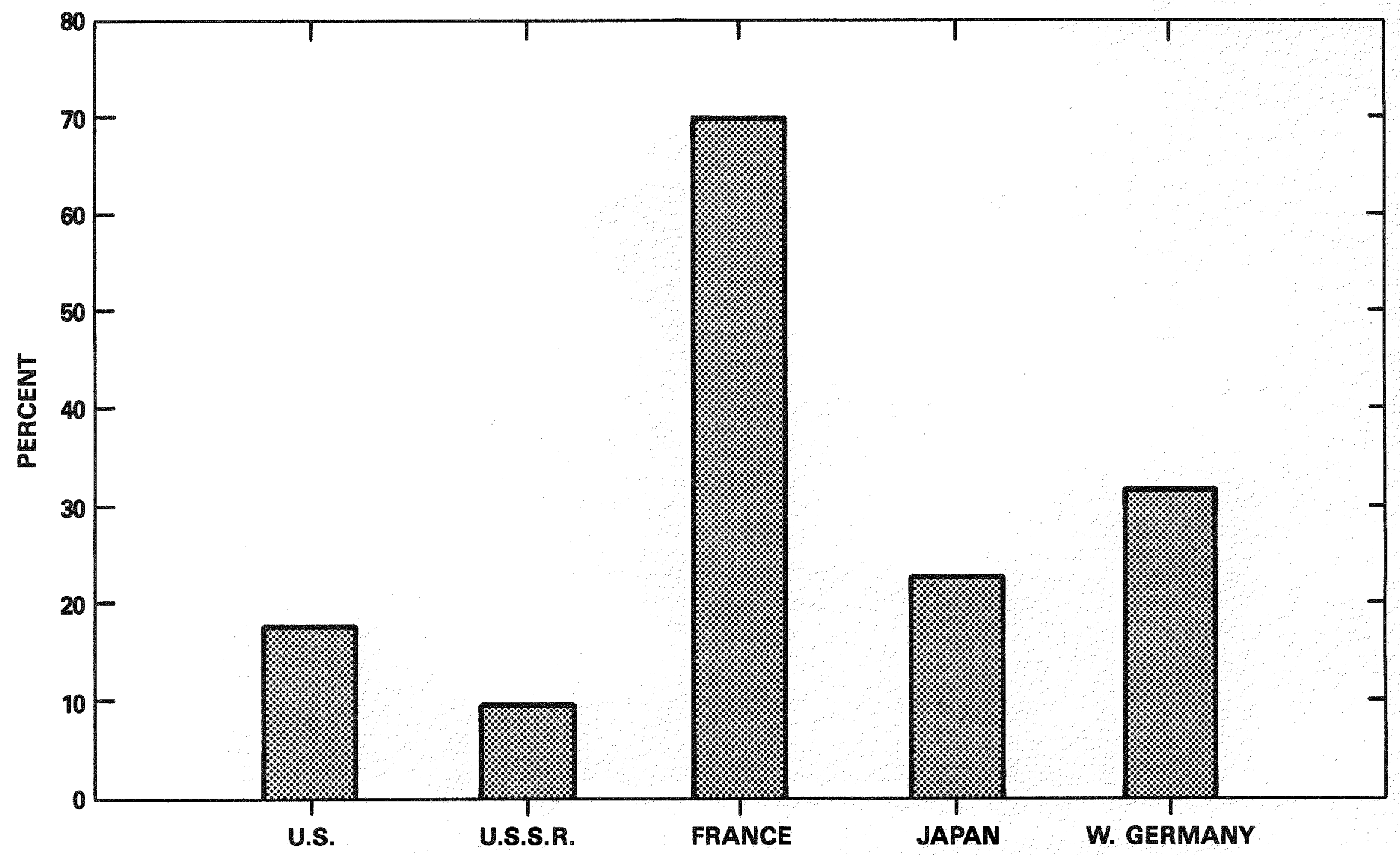




\section{NUCLEAR POWER REACTORS}

U.S. NUCLEAR POWER PLANTS GROWING OLDER (SEE EXHIBITS 10.1-10.2, PAGES 31-33)

- IN 1985 ABOUT 2\% WERE MORE THAN 20 YEARS OLD. (PRODUCING ABOUT 1\% OF THE NUCLEAR ELECTRICITY.)

- IN 1995 NEARLY 35\% WILL BE MORE THAN 20 YEARS OLD. (PRODUCING ABOUT 27\% OF THE NUCLEAR ELECTRICITY.I

- MUST ENSURE THAT CRITICAL REACTOR COMPONENTS AND SAFETY SYSTEMS WILL PROVIDE ADEQUATE RELIABILITY AS PLANTS AGE. 
DO NOT MICROFILM THIS PAGE 


\section{U.S. COMMERCIAL POWER REACTORS DISPLAYED BY AGE \& POPULATION}

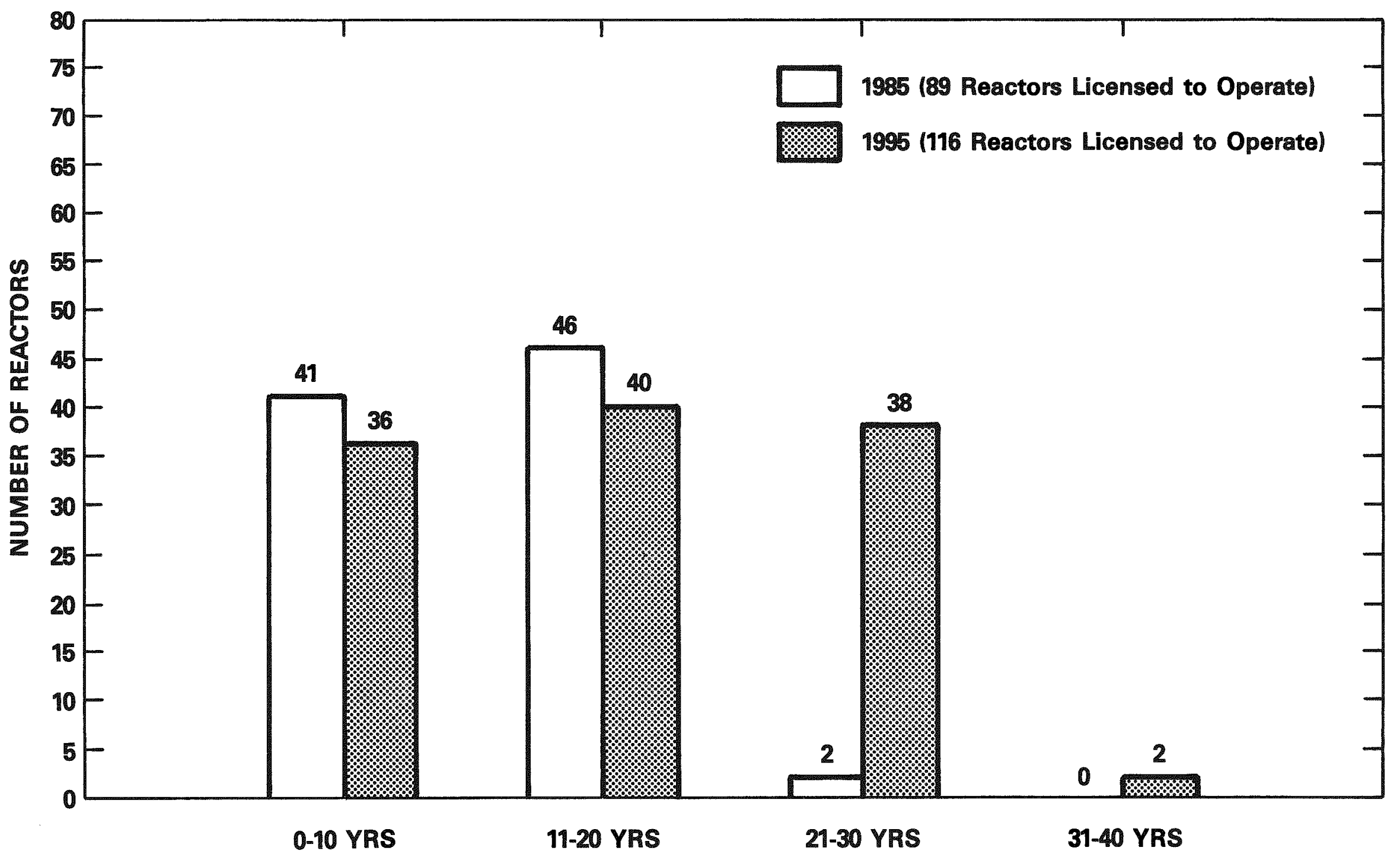


U.S. COMMERCIAL POWER REACTORS

DISPLAYED BY AGE \& ELECTRIC GENERATION

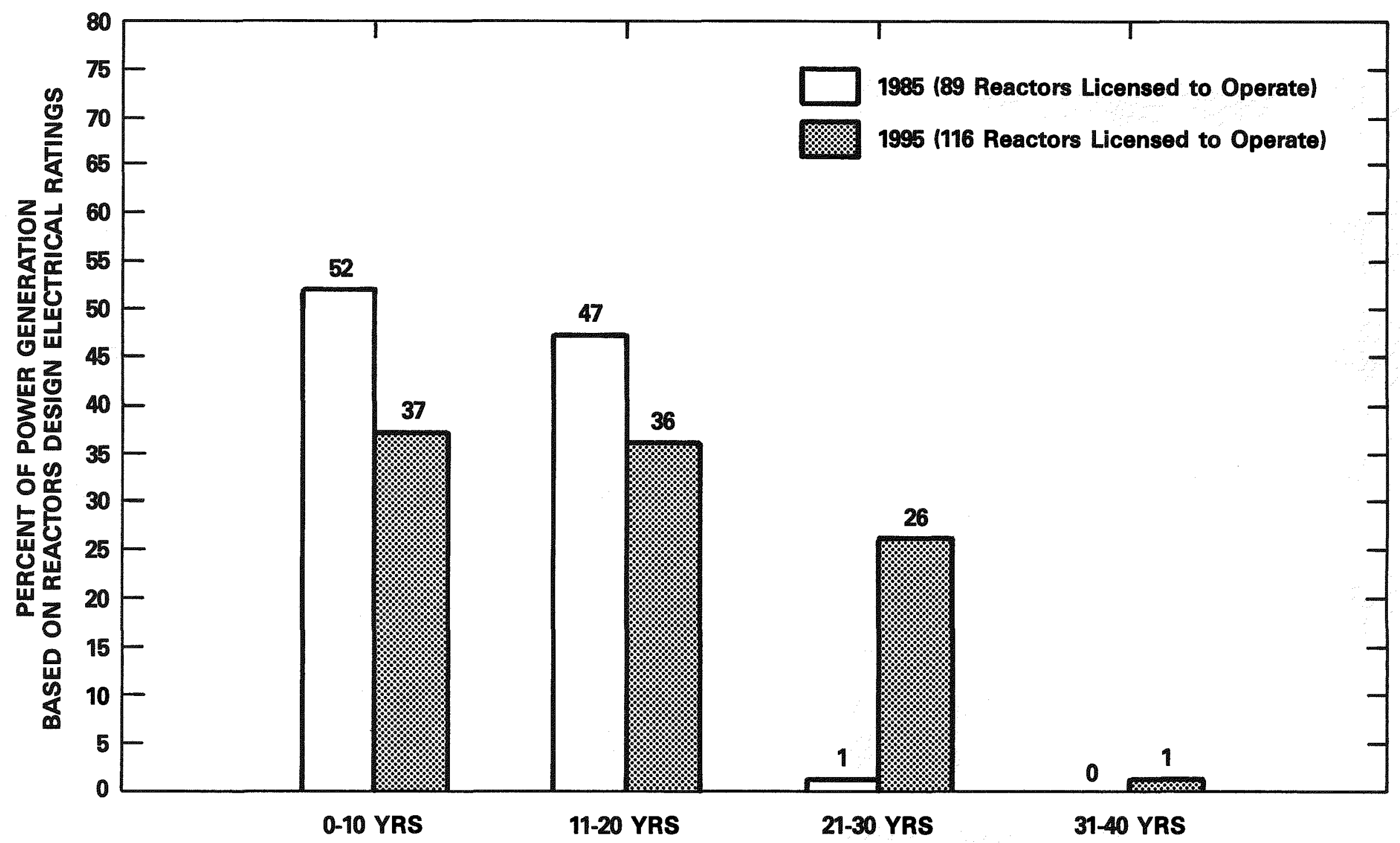




\section{U.S. COMMERCIAL POWER REACTORS BY LICENSE EXPIRATION DATE}
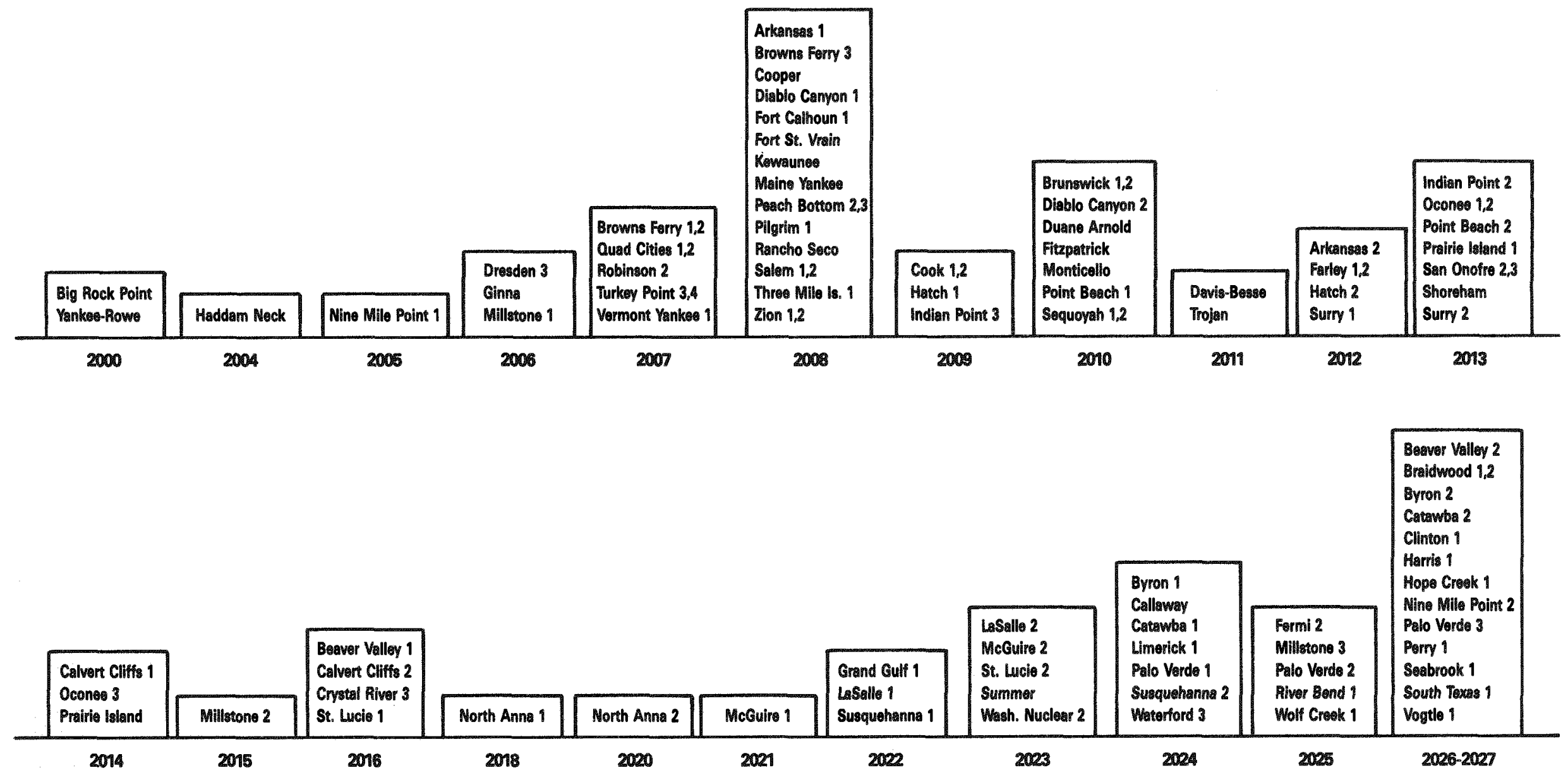

NOTE:

Dresden 2, Oyster Creak, Palisades, and San Onofre 1

have provisional operating licenses and have applied for

full-term licenses. 


\section{NONPOWER NUCLEAR REACTORS}

NONPOWER NUCLEAR REACTORS ARE DESIGNED AND UTILIZED FOR RESEARCH, TESTING AND EDUCATION

PURPOSES, I.E.

- IN THE PERFORMANCE OF RESEARCH AND TESTING IN THE AREAS OF PHYSICS, CHEMISTRY, BIOLOGY, MEDICINE, MATERIALS SCIENCES AND RELATED FIELDS.

- IN EDUCATING PEOPLE FOR NUCLEAR RELATED CAREERS IN THE POWER INDUSTRY, NATIONAL DEFENSE, AND RESEARCH AND EDUCATION.

OPERATING AND UNDER CONSTRUCTION

- 51 LICENSED TO OPERATE.

- 1 UNDER CONSTRUCTION AT THE UNIVERSITY OF TEXAS. 


\section{NONPOWER NUCLEAR REACTOR SITES}

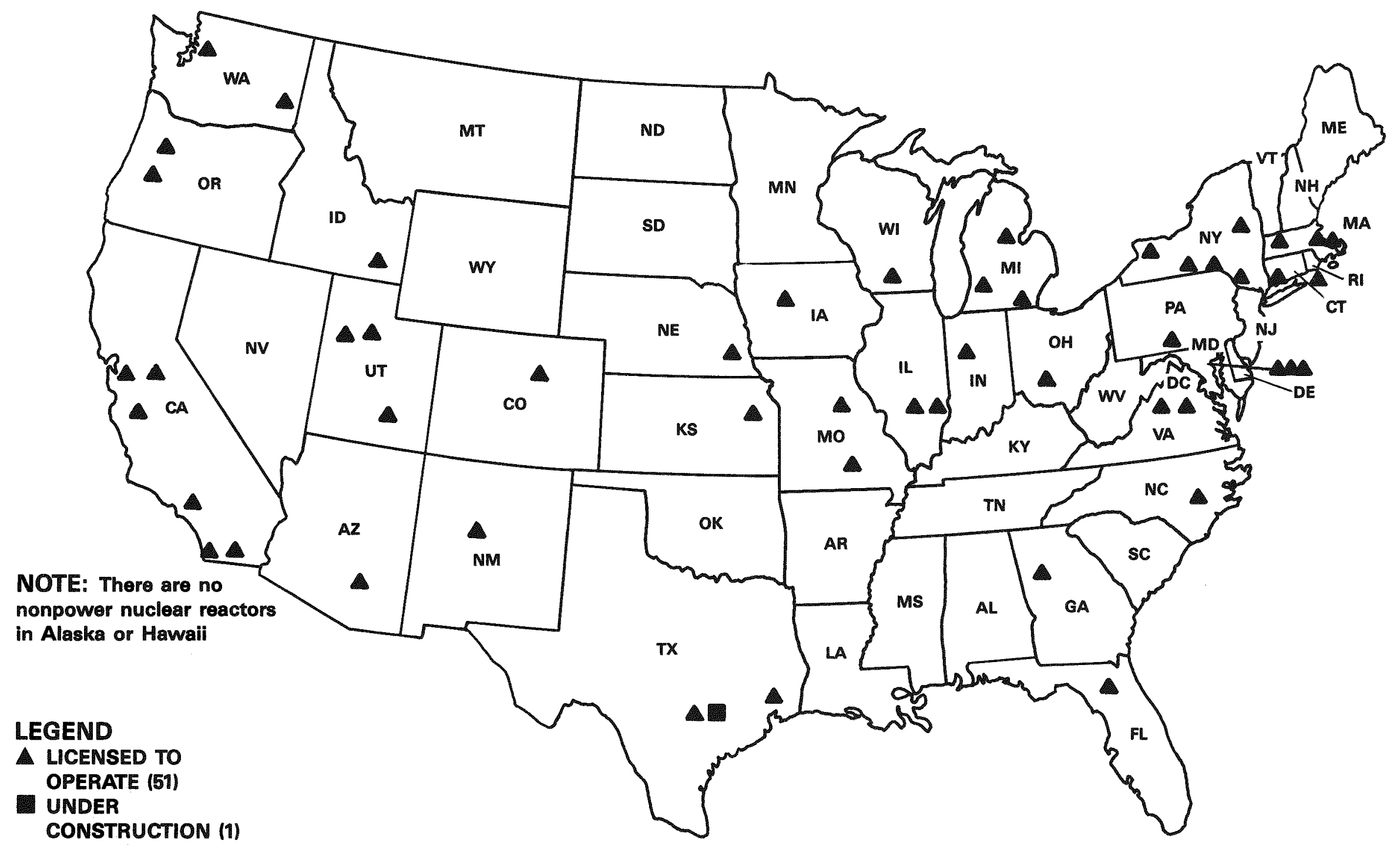




\section{FUTURE REACTOR DESIGNS}

STANDARDIZATION OF FUTURE NUCLEAR POWER PLANT DESIGNS IS AN IMPORTANT COMMISSION INITIATIVE THAT CAN SIGNIFICANTLY ENHANCE SAFETY AND RELIABILITY OF NUCLEAR POWER PLANTS

- NRC CONDUCTING DESIGN CERTIFICATION REVIEWS FOR ADVANCED DESIGNS FROM GENERAL ELECTRIC AND COMBUSTION ENGINEERING AND A PRELIMINARY DESIGN APPROVAL FOR WESTINGHOUSE.

- NRC REVIEWING THE ELECTRIC POWER RESEARCH INSTITUTE'S ADVANCED LIGHT WATER REACTOR REQUIREMENTS DOCUMENT.

- NRC CONDUCTING INITIAL LICENSABILITY REVIEW OF THREE DOE ADVANCED REACTOR CONCEPTS ITWO LIQUID METAL REACTORS AND ONE MODULAR HIGH TEMPERATURE GAS REACTOR). 


\section{NUCLEAR MATERIAL SAFETY}

FUEL CYCLE

- NRC LICENSES AND INSPECTS ALL CIVILIAN NUCLEAR FUEL FACILITIES INVOLVED IN THE PROCESSING AND FABRICATION OF URANIUM ORE INTO REACTOR FUEL.

12 MAJOR FACILITIES LICENSED to OPERATE. 
$L^{\varepsilon^{\text {obd }}}$

\author{
JDVd SHI \\ M7LOSOIN LON 00
}




\section{MAJOR FUEL CYCLE FACILITIES IN THE UNITED STATES}

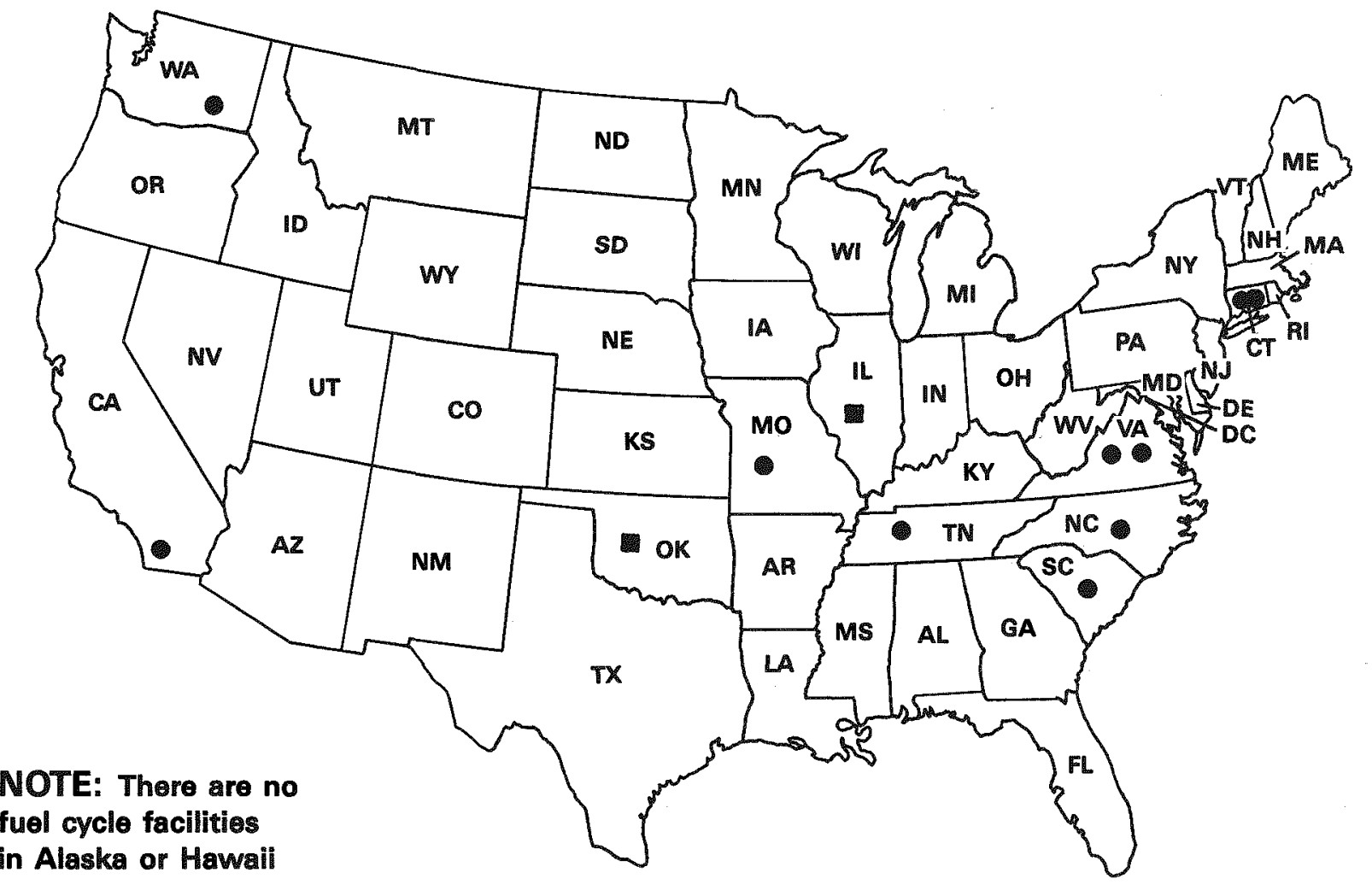

URANIUM HEXAFLUORIDE

PRODUCTION FACILITIES

- ALLIED CORPORATION METROPOLIS, IL

- SEQUOYAH FUELS CORP. SEQUOYAH, OK

URANIUM FUEL FABRICATION FACILITIES

- ga TECHNOLOgies SAN DIEGO, CA

- COMBUSTION ENGINEERING WINDSOR, CT

- UNITED NUCLEAR CORP.

UNCASVILLE, CT

- COMBUSTION ENGINEERING

HEMATITE, MO

- genERAL electric

WILMINGTON, NC

- WESTINGHOUSE

COLUMBIA, SC

- NUCLEAR fUEL SERVICEs ERWIN, TN

- BABCOCK \& WILCOX (COMM'L) LYNCHBURG, VA

- BABCOCK \& WILCOX (NAVAL) LYNCHBURG, VA

- ADVANCED NUCLEAR FUELS RICHLAND, WA 


\section{NUCLEAR MATERIAL SAFETY}

MATERIAL LICENSEES

- ABOUT 23,000 LICENSED MEDICAL, ACADEMIC, AND INDUSTRIAL USERS OF NUCLEAR MATERIAL

- 8,000 LICENSES ISSUED BY NRC.

- 15,000 LICENSES ISSUED BY THE 29 STATES THAT PARTICIPATE IN THE NRC AGREEMENT STATES PROGRAM.

- MEDICAL: OVER 7 MILLION CLINICAL PROCEDURES USING RADIOACTIVE MATERIAL PERFORMED ANNUALLY

- ABOUT 7 MILLION FOR DIAGNOSIS.

- ABOUT 200 THOUSAND FOR TREATMENT OF PATIENTS.

- ACADEMIC: USED IN UNIVERSITIES, COLLEGES, AND OTHER ACADEMIC INSTITUTIONS IN COURSE WORK AND RESEARCH.

- INDUSTRIAL: USED IN SUCH AREAS AS RADIOGRAPHY, GAUGING DEVICES, GAS CHROMOTOGRAPHY, WELL LOGGING, AND SMOKE DETECTORS.

- NRC RECEIVES FOR REVIEW NEARLY 5,200 NEW, RENEWAL, OR LICENSE AMENDMENTS FOR MATERIAL LICENSES ANNUALLY.

- NRC CONDUCTS ABOUT 2,600 HEALTH AND SAFETY INSPECTIONS OF NUCLEAR MATERIAL LICENSEES ANNUALLY. 


\section{AGREEMENT STATES}

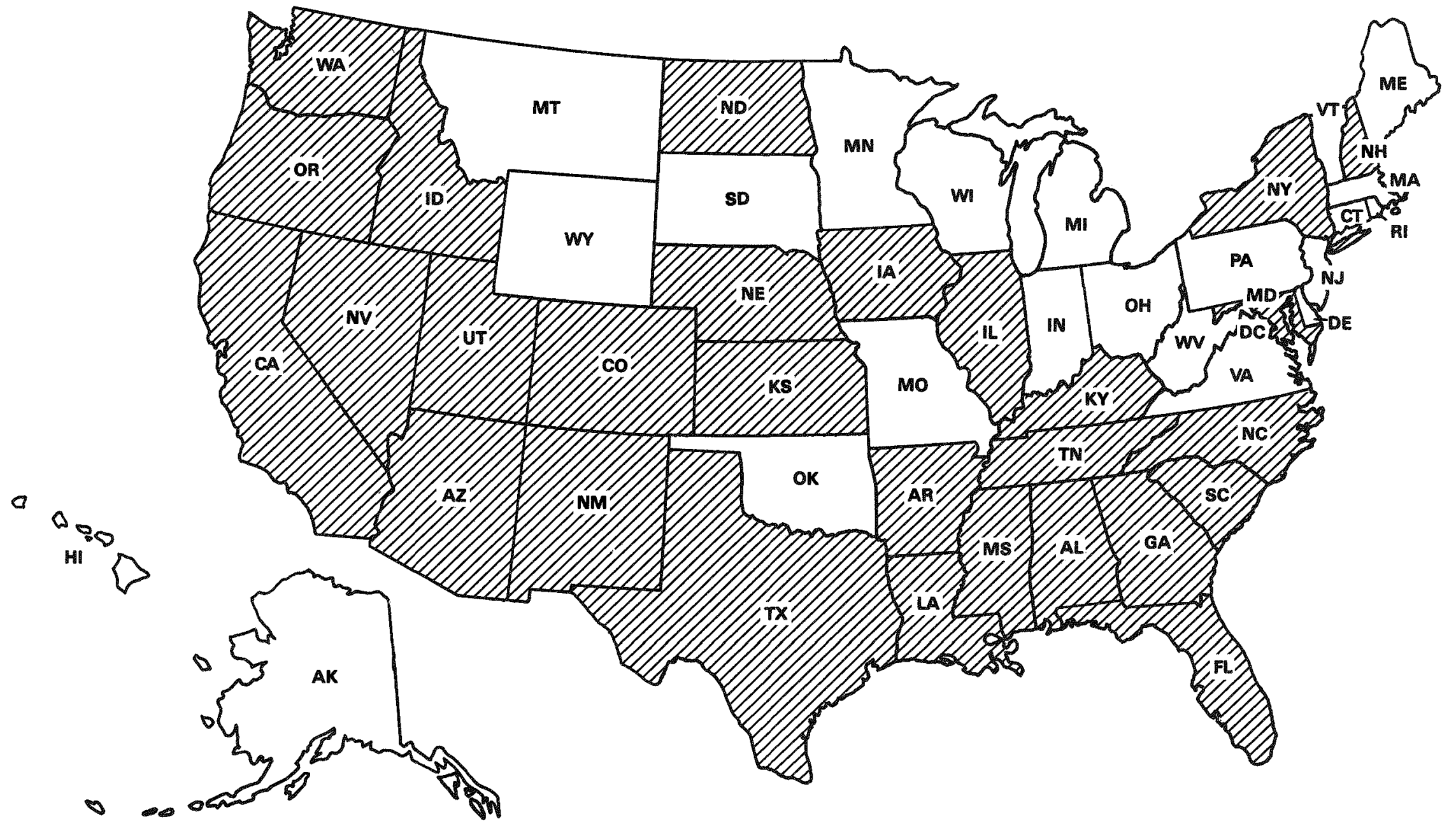

$\square$ AGREEMENT STATES (29)

A STATE THAT HAS SIGNED AN AGREEMENT WITH THE NRC, ALLOWING THE STATE TO REGULATE THE USE OF RADIOACTIVE MATERIAL WITHIN THAT STATE 


\section{NUCLEAR MATERIAL TRANSPORTATION AND SAFEGUARDS}

NRC REVIEWS, EVALUATES, AND CERTIFIES ABOUT 90 CONTAINER-DESIGN APPLICATIONS FOR TRANSPORT AND SHIPMENT OF NUCLEAR MATERIAL ANNUALLY.

NRC REVIEWS AND EVALUATES ABOUT 140 LICENSE APPLICATIONS FOR THE EXPORT OF NUCLEAR MATERIAL FROM THE UNITED STATES ANNUALLY.

NRC CONDUCTS TRANSPORT-RELATED SAFETY INSPECTIONS, QUALITY ASSURANCE INSPECTIONS, AND SAFEGUARDS INSPECTIONS OF NUCLEAR MATERIAL.

NRC AND DOE CONTINUE JOINT OPERATION OF A NATIONAL DATABASE AND INFORMATION SUPPORT SYSTEM TO TRACK MOVEMENT OF DOMESTIC AND FOREIGN NUCLEAR MATERIAL UNDER SAFEGUARDS CONTROL.

NRC INSTITUTES NEWLY DEVELOPED TECHNIQUES TO EVALUATE SECURITY SYSTEMS THROUGH TACTICAL RESPONSE EXERCISES TO EVALUATE THE OPERATIONAL EFFECTIVENESS OF LICENSEES WHO POSSESS SIGNIFICANT QUANTITIES OF STRATEGIC SPECIAL NUCLEAR MATERIAL. 


\section{HIGH LEVEL NUCLEAR WASTE DISPOSAL}

ABOUT 18,000 METRIC TONS OF NUCLEAR SPENT FUEL CURRENTLY STORED IN POOLS AT OPERATING NUCLEAR POWER PLANTS (SEE EXHIBITS 14.1-14.2, PAGES 45-46).

372,000 CUBIC METERS OF HIGH LEVEL WASTE FROM DOE ACTIVITIES CURRENTLY STORED AT HANFORD, SAVANNAH RIVER, AND IDAHO DOE SITES.

TWO OFF-SITE (I.E., NOT AT THE REACTOR SITE) SPENT FUEL STORAGE FACILITIES BUT NO LONGER ACCEPTING SPENT FUEL FOR STORAGE.

- WEST VALLEY, NEW YORK

- MORRIS, ILLINOIS

NUCLEAR WASTE POLICY ACT OF 1982 AND NUCLEAR WASTE POLICY AMENDMENTS ACT OF 1987 GIVE THE NRC REGULATORY RESPONSIBILITY FOR HIGH LEVEL WASTE DISPOSAL AND FOR LICENSING A DEEP GEOLOGICAL REPOSITORY WHICH DOE PLANS TO HAVE IN OPERATION BY 2003.

NWPAA OF 1987 NAMED YUCCA MOUNTAIN, NV, AS THE PRIME CANDIDATE SITE FOR THE HIGH LEVEL WASTE REPOSITORY AND LINKED CONSTRUCTION OF A MONITORED RETRIEVABLE STORAGE FACILITY TO NRC'S AUTHORIZING CONSTRUCTION OF A RESPOSITORY 



\section{SPENT FUEL STORED AT NUCLEAR POWER PLANTS TOTAL METRIC TONS BY STATE}

\begin{tabular}{|c|c|c|c|c|c|}
\hline STATE & 1986 & 1995* & STATE & 1986 & 1995* \\
\hline Alabama & 963 & 1826 & Mississippi & 49 & 340 \\
\hline Arizona & 0 & 594 & Missouri & 39 & 261 \\
\hline Arkansas & 329 & 637 & Nebraska & 242 & 510 \\
\hline California & 351 & 1364 & New Hampshire & 0 & $1 \pi$ \\
\hline Colorado & 0 & 11 & New Jersey & 498 & 1369 \\
\hline Connecticut & 719 & 1415 & New York & 1042 & 2203 \\
\hline Florida & 765 & 1595 & N. Carolina & 621 & 1546 \\
\hline Georgia & 342 & 1209 & Ohio & 93 & 469 \\
\hline Illinois & 2192 & 4676 & Oregon & 174 & 378 \\
\hline lowa & 129 & 262 & Pennsylvania & 964 & 2829 \\
\hline Kansas & 24 & 224 & S. Carolina & 890 & 2162 \\
\hline Louisiana & 39 & 471 & Tennessee & 159 & 802 \\
\hline Maine & 300 & 466 & Texas & 0 & 656 \\
\hline Maryland & 402 & 730 & Vermont & 246 & 391 \\
\hline Massachusetts & 330 & 520 & Virginia & 640 & 1304 \\
\hline Michigan & 673 & 1430 & Washington & 24 & 275 \\
\hline Minnesota & 390 & 747 & Wisconsin & 514 & 879 \\
\hline \multicolumn{3}{|c|}{$\begin{array}{l}\text { *Projected } \\
\text { Based On } 117 \text { Reactors }\end{array}$} & Total & 14,143 & 34,728 \\
\hline
\end{tabular}




\section{PROJECTED SPENT FUEL STORAGE CAPABILITY OF REACTORS LICENSED TO OPERATE}

Projected Year Reactor Will Lose Full Core Reserve

\begin{tabular}{|l|l|l|l|}
\hline \multicolumn{1}{c|}{} & $\begin{array}{l}\text { Calvert Cliffs 1,2 } \\
\text { Crystal River 3 } \\
\text { Farley 1 } \\
\text { Millstone 1 } \\
\text { Ocones 1,2,3 } \\
\text { Robinson 2 } \\
\text { Three Mile is. 1 }\end{array}$ & $\begin{array}{l}\text { Brunswick 1 } \\
\text { Fitzpatrick } \\
\text { Palisades } \\
\text { Vogtle 1 }\end{array}$ \\
\hline
\end{tabular}

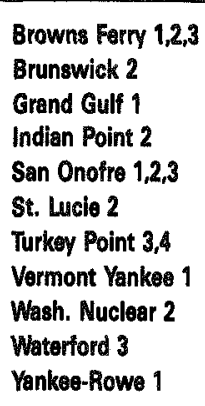

Browns Farry 1,2,3 Brunswick 2

Grand Gulf 1

Indian Point 2

San Onofre 1,2,3

St. Lucio 2

Turkey Point 3,4

Vermont Yanke 1

Wash. Nuclear 2

Waterford 3

Yankeo-Rowe 1

1992
1991

\begin{tabular}{|l|}
\hline Farley 2 \\
Fort Calhoun 1 \\
Indian Point 3 \\
Millstone 2 \\
Oyster Crook \\
Prairie lsiand 1,2 \\
Sequoyah 1,2 \\
\hline
\end{tabular}

1994

\begin{tabular}{l|}
\hline Beaver Valley 1 \\
Big Rock Point \\
Byron 1 \\
Davis-Besse 1 \\
LaSalle 1,2 \\
Peach Bottom 2 \\
Point Beach 1,2 \\
Zion 1,2 \\
\hline
\end{tabular}

1995
Arkansas 1

Fort St. Vrain

Millstone 3

Haddam Nock

Main Yankee

Nine Mile Point 1

Peach Bottom 3
Braidwood 1,2

Dresden 3

Rancho Seco 1

Salem 1

2001

1999

2000

1998

1997

1990

\begin{tabular}{|c|c|c|}
\hline Cooper Station & $\begin{array}{l}\text { Limerick } 1 \\
\text { Trojan }\end{array}$ & $\begin{array}{l}\text { Fermi } 2 \\
\text { Palo Vorde } 12\end{array}$ \\
\hline
\end{tabular}

2002
2004
Beaver Valloy 2

\section{Callaway}

Catawba 1,2

Clinton 1

Diablo Canyon 1,2

Harris 1

MoGuire 1,2

Nine Mile Point 2

Quad Cities 1,2

Palo Verdo 3

Parry 1

River Bend 1

Salem 2

South Texas 1

St. Lucie 1

Summer 1

Surry 1,2

Wolf Crook 1 


\section{LOW LEVEL NUCLEAR WASTE DISPOSAL}

1.8 MILLION CUBIC FEET OF LOW LEVEL RADIOACTIVE WASTE GENERATED IN 1987.

- 3 ACTIVE LICENSED SITES (SEE EXHIBIT 16, PAGE 50).

- BARNWELL, SOUTH CAROLINA.

- BEATTY, NEVADA.

- HANFORD, WASHINGTON.

LOW LEVEL RADIOACTIVE WASTE POLICY AMENDMENTS ACT OF 1985 AUTHORIZED

- FORMATION OF REGIONAL COMPACTS: 9 COMPACTS NOW ACTIVE (SEE EXHIBIT 17, AGE 51).

- EXCLUSION OF WASTE GENERATED OUTSIDE A COMPACT.

- EACH COMPACT to DESIgNATE 1 ACTIVE SITE.

- SYSTEM OF MILESTONES, INCENTIVES, AND PENALTIES TO ENSURE THAT STATES AND COMPACTS WILL BE RESPONSIBLE FOR THEIR OWN WASTE IN 1993. 
DO NOT MICROFLM THIS PAGE 


\section{LOW LEVEL RADIOACTIVE WASTE BY SOURCE}

1.8M CU. FT. GENERATED ANNUALLY IN THE U.S.

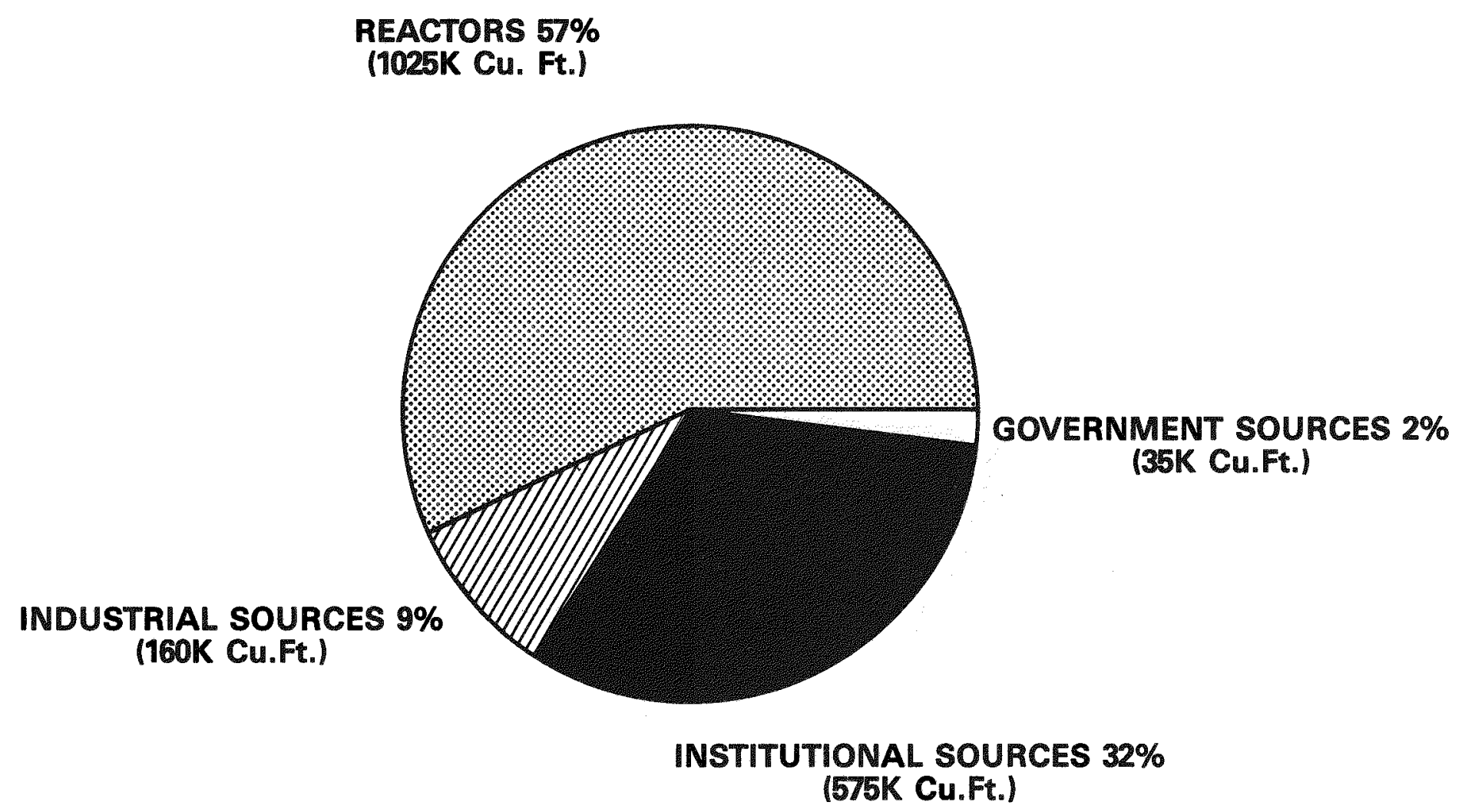




\section{COMMERCIAL LOW LEVEL WASTE DISPOSAL SITES}

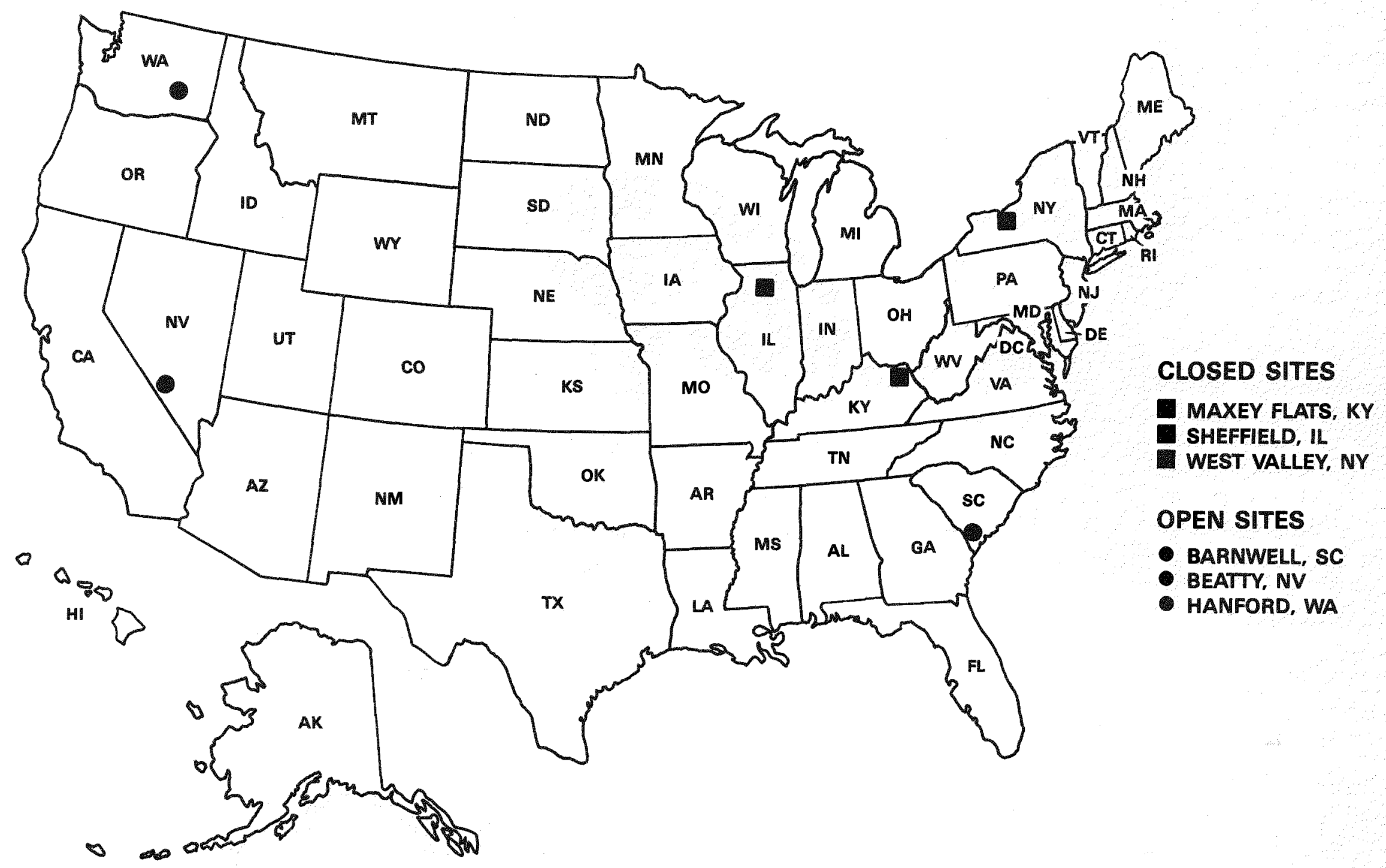




\section{REGIONAL LOW LEVEL WASTE COMPACTS}

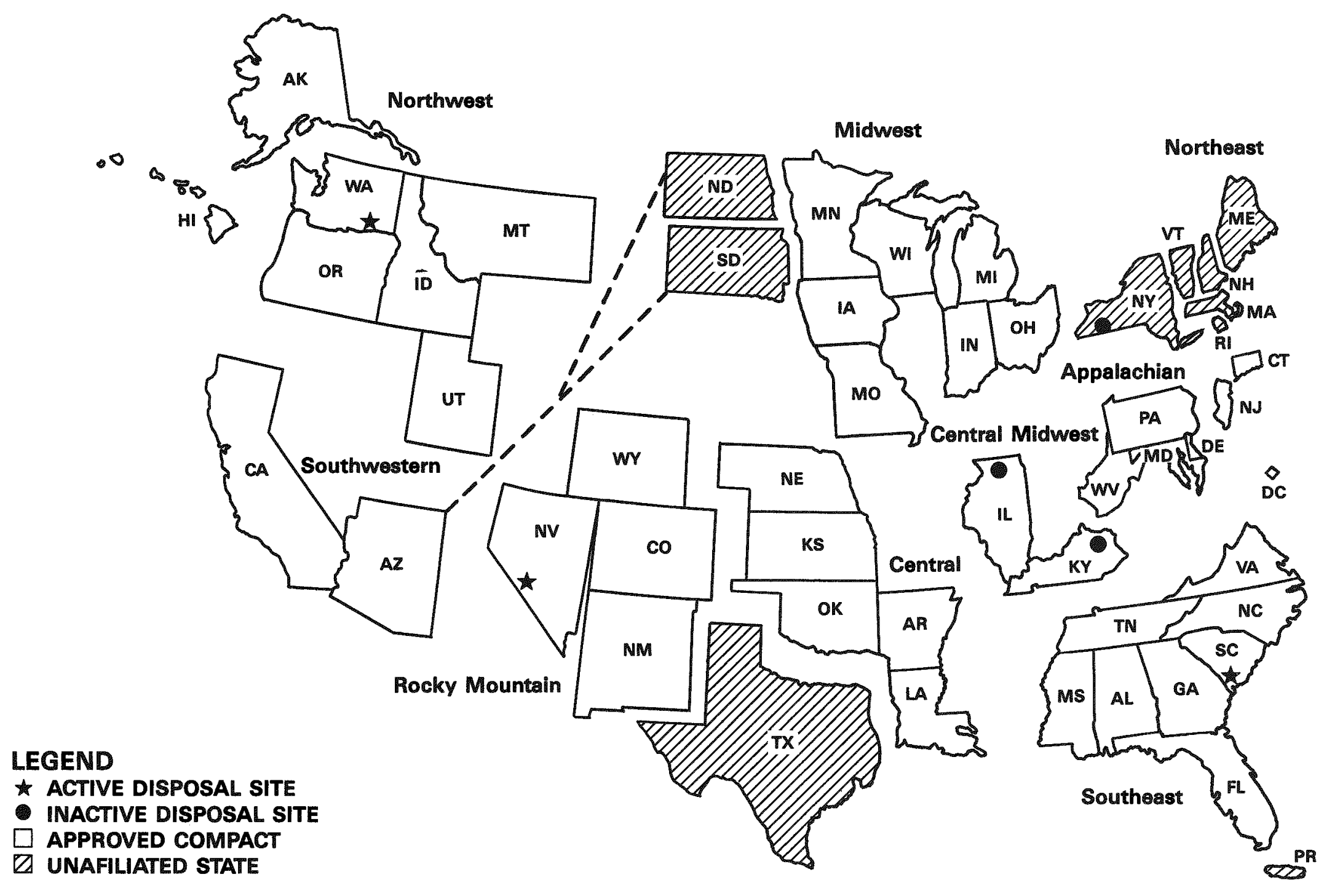




\section{DO NOT MICROFILM THIS PAGE}


PART II: NRC LICENSEE AND FOREIGN POWER REACTOR DATA 


\section{U.S. COMMERCIAL NUCLEAR POWER REACTORS}

\begin{tabular}{|l|l||l|l|}
\hline \multicolumn{3}{|c|}{ ABBREVIATIONS USED IN THIS SECTION } \\
\hline AE: & Architect-Engineer & DPR: & Demonstration Power Reactor \\
AEP: & American Electric Power & DUKE: & Duke Power \\
B\&R & Burns and Roe & EBSO: & Ebasco \\
B\&W: & Babcock \& Wilcox & EXP. DATE: & Expiration Date of OL \\
BALD: & Baldwin Associates & FLUR: & Fluor Pioneer \\
BECH: & Bechtel & G\&H: & Gibbs and Hill \\
BRRT: & Brown and Root & GAC: & General Atomic \\
BWR: & Boiling Water Reactor & GE: & General Electric \\
COMB: & Combustion Engineering & GHDR: & Gibbs \& Hill \& Durham \& Richardson \\
COMM: OP:: & Date of Commercial Operation & GIL: & Gilbert Associates \\
CONST: & Constructor & GPO: & Georgia Power \\
CONTYPE & Containment Type & HTGR: & High Temperature Gas-Cooled Reactor \\
DRYAMB & Dry, Ambient Pressure & JONES: & J. A. Jones \\
DRYSUB & Dry, Subatmospheric & KAIS: & Kaiser Engineers \\
GASCLD & Gas Cooled & LIC. TYPE: & License Type \\
ICECND & Wet, Ice Condenser & CP: & Construction Permit \\
MARK 1 & Wet, Mark I & OL-FL: & Operating License - Fuel Load \\
MARK 2 & Wet, Mark II & OL-FP: & Operating License - Full Power \\
MARK 3 & Wet, Mark III & OL-LP: & Operating License - Low Power \\
CP ISSUED: & Date of Construction Permit Issuance & OL-P: & Provisional Operating License \\
CPPR: & Construction Permit Power Reactor & MDC: & Maximum Dependable Capacity - Net \\
CWE: & Commonwealth Edison & MWE: & Megawatts Electrical \\
DANI: & Daniel International & MWT: & Megawatts Thermal \\
DBDB: & Duke and Bechtel & NIAG: & Niagara Mohawk \\
DER: & Design Electric Rating & NPF: & Nuclear Power Facility \\
\hline
\end{tabular}




\section{U.S. COMMERCIAL NUCLEAR POWER REACTORS}

\begin{tabular}{|l|l||l|l|}
\hline \multicolumn{3}{|c|}{ ABBREVIATIONS USED IN THIS SECTION } \\
\hline NSP: & Northern States & TVA: & Tennessee Valley Authority \\
NSSS: & Nuclear Steam System Supplier \& Design Type & UEC: & United Engineering \\
1: & GE Type 1 & WDCO: & Westinghouse Development \\
2: & GE Type 2 & \\
3: & GE Type 3 & \\
4: & GE Type 4 & \\
5: & GE Type 5 & \\
6: & GE Type 6 & \\
2LP: & West Two-Loop & \\
3LP: & West. Three-Loop & \\
4LP: & West. Four-Loop & \\
CE: & Combustion Engineering & \\
CE80: & CE Standard Design & \\
LLP: & B\&W Lowered Loop & \\
RLP: & B\&W Raised Loop & \\
OL ISSUED: & Date of Most Recent OL & \\
PG\&E: & Pacific Gas and Electric & \\
PSE: & Pioneer Services and Engineering & \\
PUBS: & Public Service Electric and Gas & \\
PWR: & Pressurized Water Reactor & \\
S\&L: & Sargent and Lundy & \\
S\&W: & Stone and Webster & \\
SBEC: & Southern Services and Bechtel & \\
SSI: & Southern Services & \\
\hline
\end{tabular}




\section{U.S. COMMERCIAL NUCLEAR POWER REACTORS}

\begin{tabular}{|c|c|c|c|c|c|c|c|}
\hline $\begin{array}{c}\text { UNIT } \\
\text { OPERATING UTILITY } \\
\text { LOCATION } \\
\text { DOCKET NUMBER }\end{array}$ & $\begin{array}{c}\text { NRC } \\
\text { REGION }\end{array}$ & $\begin{array}{l}\text { CONTYPE } \\
\text { NSSS } \\
\text { AE } \\
\text { CONST }\end{array}$ & $\begin{array}{l}\text { LIC } \\
\text { MWT }\end{array}$ & $\begin{array}{l}\text { MDC } \\
\text { NET } \\
\text { OR } \\
\text { DER }\end{array}$ & $\begin{array}{l}\text { CP ISSUED } \\
\text { OL ISSUED } \\
\text { COMM. OP } \\
\text { EXP. DATE }\end{array}$ & $\begin{array}{c}\text { LICENSE } \\
\text { TYPE } \\
8 \\
\text { NUMBER }\end{array}$ & REMARKS \\
\hline $\begin{array}{l}\text { Arkansas } 1 \\
\text { Arkansas Power \& Light Co. } \\
6 \text { MI WNW of Russellville, AR } \\
050-00313\end{array}$ & IV & $\begin{array}{l}\text { PWR-DRYAMB } \\
\text { BEW LLP } \\
\text { BECH } \\
\text { BECH }\end{array}$ & 2568 & $\begin{array}{c}0836 \\
\text { (MDC) }\end{array}$ & $\begin{array}{l}12 / 06 / 1968 \\
05 / 21 / 1974 \\
12 / 19 / 1974 \\
12 / 06 / 2008\end{array}$ & $\begin{array}{l}\text { OL-FP } \\
\text { DPR-51 }\end{array}$ & \\
\hline $\begin{array}{l}\text { Arkansas } 2 \\
\text { Arkansas Power \& Light Co. } \\
6 \mathrm{MI} \text { WNW of Russellville, AR } \\
050-00368\end{array}$ & IV & $\begin{array}{l}\text { PWR-DRYAMB } \\
\text { COMB CE } \\
\text { BECH } \\
\text { BECH }\end{array}$ & 2815 & $\begin{array}{c}0858 \\
\text { (MDC) }\end{array}$ & $\begin{array}{l}12 / 06 / 1972 \\
12 / 14 / 1978 \\
04 / 26 / 1980 \\
12 / 06 / 2012\end{array}$ & $\begin{array}{l}\text { OL-FP } \\
\text { NPF-6 }\end{array}$ & \\
\hline $\begin{array}{l}\text { Beaver Valley } 1 \\
\text { Duquesne Light Co. } \\
17 \mathrm{MI} W \text { of McCandless, PA } \\
05-00334\end{array}$ & I & $\begin{array}{l}\text { PWR-DRYSUB } \\
\text { WEST 3LP } \\
\text { S\&W } \\
\text { S\&W }\end{array}$ & 2652 & $\begin{array}{c}0810 \\
\text { (MDC) }\end{array}$ & $\begin{array}{l}06 / 26 / 1970 \\
07 / 02 / 1976 \\
10 / 01 / 1976 \\
01 / 29 / 2016\end{array}$ & $\begin{array}{l}\text { OL-FP } \\
\text { DPR-66 }\end{array}$ & \\
\hline $\begin{array}{l}\text { Beaver Valley } 2 \\
\text { Duquesne Light Co. } \\
17 \mathrm{MI} \text { W of McCandless, PA } \\
050-00412\end{array}$ & 1 & $\begin{array}{l}\text { PWR-DRYSUB } \\
\text { WEST 3LP } \\
\text { S\&W } \\
\text { S\&W }\end{array}$ & 2652 & $\begin{array}{c}0833 \\
\text { (MDC) }\end{array}$ & $\begin{array}{l}05 / 03 / 1974 \\
08 / 14 / 1987 \\
11 / 17 / 1987 \\
05 / 27 / 2027\end{array}$ & $\begin{array}{l}\text { OL-FP } \\
\text { NPF-73 }\end{array}$ & \\
\hline $\begin{array}{l}\text { Bellefonte } 1 \\
\text { Tennessee Valley Authority } \\
6 \mathrm{MI} \text { NE of Scottsboro, AL } \\
050-00438\end{array}$ & II & $\begin{array}{l}\text { PWR-DRYAMB } \\
\text { B\&W r RLP } \\
\text { TVA } \\
\text { TVA }\end{array}$ & 0000 & $\begin{array}{c}1235 \\
\text { (DER) }\end{array}$ & $12 / 24 / 1974$ & $\begin{array}{l}\text { CP } \\
\text { CPPR-122 }\end{array}$ & $\begin{array}{l}\text { Construction } \\
\text { Halted }\end{array}$ \\
\hline $\begin{array}{l}\text { Bellefonte } 2 \\
\text { Tennessee Valley Authority } \\
6 \text { MI NE of Scottsboro, AL } \\
050-00439\end{array}$ & II & $\begin{array}{l}\text { PWR-DRYAMB } \\
\text { B\&W RLP } \\
\text { TVA } \\
\text { TVA }\end{array}$ & 0000 & $\begin{array}{l}1235 \\
\text { (DER) }\end{array}$ & $12 / 24 / 1974$ & $\begin{array}{l}\text { CP } \\
\text { CPPR-123 }\end{array}$ & $\begin{array}{l}\text { Construction } \\
\text { Halted }\end{array}$ \\
\hline
\end{tabular}




\section{U.S. COMMERCIAL NUCLEAR POWER REACTORS}

\begin{tabular}{|c|c|c|c|c|c|c|c|}
\hline $\begin{array}{c}\text { UNIT } \\
\text { OPERATING UTILITY } \\
\text { LOCATION } \\
\text { DOCKET NUMBER }\end{array}$ & $\begin{array}{c}\text { NRC } \\
\text { REGION }\end{array}$ & $\begin{array}{l}\text { CONTYPE } \\
\text { NSSS } \\
\text { AE } \\
\text { CONST }\end{array}$ & $\begin{array}{l}\text { LIC } \\
\text { MWT }\end{array}$ & $\begin{array}{l}\text { MDC } \\
\text { NET } \\
\text { OR } \\
\text { DER }\end{array}$ & $\begin{array}{l}\text { CP ISSUED } \\
\text { OL ISSUED } \\
\text { COMM. OP } \\
\text { EXP. DATE }\end{array}$ & $\begin{array}{c}\text { LICENSE } \\
\text { TYPE } \\
\& \\
\text { NUMBER }\end{array}$ & REMARKS \\
\hline $\begin{array}{l}\text { Big Rock Polnt } \\
\text { Consumers Power Co. } \\
4 \text { MI NE of Charlevoix, MI } \\
\text { C-00155 }\end{array}$ & III & $\begin{array}{l}\text { BWR-DRYAMB } \\
\text { GE } \\
\text { BECH } \\
\text { BECH }\end{array}$ & 0240 & $\begin{array}{c}0069 \\
\text { (MDC) }\end{array}$ & $\begin{array}{l}05 / 31 / 1960 \\
05 / 01 / 1964 \\
03 / 29 / 1963 \\
05 / 31 / 2000\end{array}$ & $\begin{array}{l}\text { OL-FP } \\
\text { DPR-6 }\end{array}$ & \\
\hline $\begin{array}{l}\text { Braidwood } 1 \\
\text { Commonwealth Edison Co. } \\
24 \text { MI SSW of Joilet, IL } \\
\text { C.0-00456 }\end{array}$ & III & $\begin{array}{l}\text { PWR-DRYAMB } \\
\text { WEST 4LP } \\
\text { S\&L } \\
\text { CWE }\end{array}$ & 3411 & $\begin{array}{c}1120 \\
\text { (MDC) }\end{array}$ & $\begin{array}{l}12 / 31 / 1975 \\
07 / 02 / 1987 \\
07 / 29 / 1988 \\
10 / 17 / 2026\end{array}$ & $\begin{array}{l}\text { OL-FP } \\
\text { NPF-72 }\end{array}$ & \\
\hline $\begin{array}{l}\text { Braidwood } 2 \\
\text { Commonwealth Edison Co. } \\
24 \text { MI SSW of Joilet, IL } \\
\text { C-00457 }\end{array}$ & III & $\begin{array}{l}\text { PWR-DRYAMB } \\
\text { WEST 4LP } \\
\text { S\&L } \\
\text { CWE }\end{array}$ & 3411 & $\begin{array}{c}1120 \\
\text { (MDC) }\end{array}$ & $\begin{array}{l}12 / 31 / 1975 \\
05 / 20 / 1988 \\
10 / 17 / 1988 \\
12 / 18 / 2027\end{array}$ & $\begin{array}{l}\text { OL-FP } \\
\text { NPF-T7 }\end{array}$ & \\
\hline $\begin{array}{l}\text { Browns Ferry } 1 \\
\text { Tennessee Valley Authority } \\
10 \text { MI NW of Decatur, AL } \\
\text { C } 0-00259\end{array}$ & II & $\begin{array}{l}\text { BWR-MARK1 } \\
\text { GE } \\
\text { TVA } \\
\text { TVA }\end{array}$ & 3293 & $\begin{array}{c}1065 \\
\text { (MDC) }\end{array}$ & $\begin{array}{l}05 / 10 / 1967 \\
12 / 20 / 1973 \\
08 / 01 / 1974 \\
05 / 10 / 2007\end{array}$ & $\begin{array}{l}\text { OL-FP } \\
\text { DPR-33 }\end{array}$ & \\
\hline $\begin{array}{l}\text { Browns Ferry } 2 \\
\text { Tennessee Valley Authority } \\
10 \text { MI NW of Decatur, AL } \\
60-00260\end{array}$ & II & $\begin{array}{l}\text { BWR-MARK1 } \\
\text { GE } \\
\text { TVA } \\
\text { TVA }\end{array}$ & 3293 & $\begin{array}{c}1065 \\
\text { (MDC) }\end{array}$ & $\begin{array}{l}05 / 10 / 1967 \\
08 / 02 / 1974 \\
03 / 01 / 1975 \\
05 / 10 / 2007\end{array}$ & $\begin{array}{l}\text { OL-FP } \\
\text { DPR-52 }\end{array}$ & \\
\hline $\begin{array}{l}\text { Browns Ferry } 3 \\
\text { Tennessee Valley Authority } \\
10 \text { MI NW of Decatur, AL } \\
\text { C-00296 }\end{array}$ & II & $\begin{array}{l}\text { BWR-MARK1 } \\
\text { GE } \\
\text { TVA } \\
\text { TVA }\end{array}$ & 3293 & $\begin{array}{c}1065 \\
\text { (MDC) }\end{array}$ & $\begin{array}{l}07 / 31 / 1968 \\
08 / 18 / 1976 \\
03 / 01 / 1977 \\
07 / 21 / 2008\end{array}$ & $\begin{array}{l}\text { OL-FP } \\
\text { DPR-68 }\end{array}$ & \\
\hline
\end{tabular}




\section{U.S. COMMERCIAL NUCLEAR POWER REACTORS}

\begin{tabular}{|c|c|c|c|c|c|c|c|}
\hline $\begin{array}{l}\text { UNIT } \\
\text { OPERATING UTILITY } \\
\text { LOCATION } \\
\text { DOCKET NUMBER }\end{array}$ & $\begin{array}{c}\text { NRC } \\
\text { REGION }\end{array}$ & $\begin{array}{l}\text { CONTYPE } \\
\text { NSSS } \\
\text { AE } \\
\text { CONST }\end{array}$ & $\begin{array}{l}\text { LIC } \\
\text { MWT }\end{array}$ & $\begin{array}{l}\text { MDC } \\
\text { NET } \\
\text { OR } \\
\text { DER }\end{array}$ & $\begin{array}{l}\text { CP ISSUED } \\
\text { OL ISSUED } \\
\text { COMM. OP } \\
\text { EXP. DATE }\end{array}$ & $\begin{array}{l}\text { LICENSE } \\
\text { TYPE } \\
\text { \& } \\
\text { NUMBER }\end{array}$ & REMARKS \\
\hline $\begin{array}{l}\text { Brunswick } 1 \\
\text { Carolina Power \& Light Co. } \\
2 \text { MI N of Southport, NC } \\
050-00325\end{array}$ & II & $\begin{array}{l}\text { BWR-MARK1 } \\
\text { GE } \\
\text { UEC } \\
\text { BRRT }\end{array}$ & 2436 & $\begin{array}{c}0790 \\
\text { (MDC) }\end{array}$ & $\begin{array}{l}02 / 07 / 1970 \\
11 / 12 / 1976 \\
03 / 18 / 1977 \\
02 / 07 / 2010\end{array}$ & $\begin{array}{l}\text { OL-FP } \\
\text { DPR-71 }\end{array}$ & \\
\hline $\begin{array}{l}\text { Brunswick } 2 \\
\text { Carolina Power \& Light Co. } \\
2 \text { MI N of Southport, NC } \\
050-00324\end{array}$ & II & $\begin{array}{l}\text { BWR-MARK1 } \\
\text { GE } \\
\text { UEC } \\
\text { BRRT }\end{array}$ & 2436 & $\begin{array}{c}0790 \\
\text { (MDC) }\end{array}$ & $\begin{array}{l}02 / 07 / 1970 \\
12 / 27 / 1974 \\
11 / 03 / 1975 \\
02 / 06 / 2010\end{array}$ & $\begin{array}{l}\text { OL-FP } \\
\text { DPR-62 }\end{array}$ & \\
\hline $\begin{array}{l}\text { Byron } 1 \\
\text { Commonwealth Edison Co. } \\
17 \mathrm{MI} \text { SW of Rockford, IL } \\
050-00454\end{array}$ & III & $\begin{array}{l}\text { PWR-DRYAMB } \\
\text { WEST 4LP } \\
\text { SEL } \\
\text { CWE }\end{array}$ & 3411 & $\begin{array}{l}1105 \\
\text { (MDC) }\end{array}$ & $\begin{array}{l}12 / 31 / 1975 \\
02 / 14 / 1985 \\
09 / 16 / 1985 \\
10 / 31 / 2024\end{array}$ & $\begin{array}{l}\text { OL-FP } \\
\text { NPF-37 }\end{array}$ & \\
\hline $\begin{array}{l}\text { Byron } 2 \\
\text { Commonwealth Edison Co. } \\
17 \mathrm{MI} \text { SW of Rockford, IL } \\
050-00455\end{array}$ & III & $\begin{array}{l}\text { PWR-DRYAMB } \\
\text { WEST } \quad 4 \text { P } \\
\text { S\&L } \\
\text { CWE }\end{array}$ & 3411 & $\begin{array}{c}1105 \\
\text { (MDC) }\end{array}$ & $\begin{array}{l}12 / 31 / 1975 \\
01 / 30 / 1987 \\
08 / 21 / 1987 \\
11 / 06 / 2026\end{array}$ & $\begin{array}{l}\text { OL-FP } \\
\text { NPF-66 }\end{array}$ & \\
\hline $\begin{array}{l}\text { Callaway } \\
\text { Union Electric Co. } \\
10 \text { Mi SE of Fulton, MO } \\
050-00483\end{array}$ & III & $\begin{array}{l}\text { PWR-DRYAMB } \\
\text { WEST } \quad 4 \text { LP } \\
\text { BECH } \\
\text { DANI }\end{array}$ & 3565 & $\begin{array}{c}1145 \\
\text { (MDC) }\end{array}$ & $\begin{array}{l}04 / 16 / 1976 \\
10 / 18 / 1984 \\
12 / 19 / 1984 \\
10 / 18 / 2024\end{array}$ & $\begin{array}{l}\text { OL-FP } \\
\text { NPF-30 }\end{array}$ & \\
\hline $\begin{array}{l}\text { Calvert Cliffs } 1 \\
\text { Baltimore Gas \& Electric } \\
40 \mathrm{MI} S \text { of Annapolis, MD } \\
050-00317\end{array}$ & 1 & $\begin{array}{l}\text { PWR-DRYAMB } \\
\text { COMB CE } \\
\text { BECH } \\
\text { BECH }\end{array}$ & 2700 & $\begin{array}{c}0825 \\
\text { (MDC) }\end{array}$ & $\begin{array}{l}07 / 07 / 1969 \\
07 / 31 / 1974 \\
05 / 08 / 1975 \\
07 / 31 / 2014\end{array}$ & $\begin{array}{l}\text { OL-FP } \\
\text { DPR-53 }\end{array}$ & \\
\hline
\end{tabular}




\section{U.S. COMMERCIAL NUCLEAR POWER REACTORS}

\begin{tabular}{|c|c|c|c|c|c|c|c|}
\hline $\begin{array}{c}\text { UNIT } \\
\text { OPERATING UTILITY } \\
\text { LOCATION } \\
\text { DOCKET NUMBER }\end{array}$ & $\begin{array}{c}\text { NRC } \\
\text { REGION }\end{array}$ & $\begin{array}{l}\text { CONTYPE } \\
\text { NSSS } \\
\text { AE } \\
\text { CONST }\end{array}$ & $\begin{array}{l}\text { LIC } \\
\text { MWT }\end{array}$ & $\begin{array}{l}\text { MDC } \\
\text { NET } \\
\text { OR } \\
\text { DER }\end{array}$ & $\begin{array}{l}\text { CP ISSUED } \\
\text { OL ISSUED } \\
\text { COMM. OP } \\
\text { EXP. DATE }\end{array}$ & $\begin{array}{c}\text { LICENSE } \\
\text { TYPE } \\
\& \\
\text { NUMBER }\end{array}$ & REMARKS \\
\hline $\begin{array}{l}\text { Calvert Cliffs } 2 \\
\text { Baltimore Gas \& Electric CO. } \\
40 \text { MI S of Annapolis, MD } \\
060-00318\end{array}$ & 1 & $\begin{array}{l}\text { PWR-DRYAMB } \\
\text { COMB CE } \\
\text { BECH } \\
\text { BECH }\end{array}$ & 2700 & $\begin{array}{c}0825 \\
\text { (MDC) }\end{array}$ & $\begin{array}{l}07 / 07 / 1969 \\
11 / 30 / 1976 \\
04 / 01 / 1977 \\
08 / 31 / 2016\end{array}$ & $\begin{array}{l}\text { OL-FP } \\
\text { DPR-69 }\end{array}$ & \\
\hline $\begin{array}{l}\text { Catawba } 1 \\
\text { Duke Power Co. } \\
6 \text { MI NNW of Rock Hill, SC } \\
050-00413\end{array}$ & II & $\begin{array}{l}\text { PWR-ICECND } \\
\text { WEST } \quad 4 L P \\
\text { DUKE } \\
\text { DUKE }\end{array}$ & 3411 & $\begin{array}{c}1129 \\
\text { (MDC) }\end{array}$ & $\begin{array}{l}08 / 07 / 1975 \\
01 / 17 / 1985 \\
06 / 29 / 1985 \\
12 / 06 / 2024\end{array}$ & $\begin{array}{l}\text { OL-FP } \\
\text { NPF-35 }\end{array}$ & \\
\hline $\begin{array}{l}\text { Catawba } 2 \\
\text { Duke Power Co. } \\
6 \text { MI NNW of Rock Hill, SC } \\
060-00414\end{array}$ & II & $\begin{array}{l}\text { PWR-ICECND } \\
\text { WEST 4LP } \\
\text { DUKE } \\
\text { DUKE }\end{array}$ & 3411 & $\begin{array}{c}1129 \\
\text { (MDC) }\end{array}$ & $\begin{array}{l}08 / 07 / 1975 \\
05 / 15 / 1986 \\
08 / 19 / 1986 \\
02 / 24 / 2026\end{array}$ & $\begin{array}{l}\text { OL-FP } \\
\text { NPF-52 }\end{array}$ & \\
\hline $\begin{array}{l}\text { Clinton } \\
\text { Illinois Power Co. } \\
6 \text { MI E of Clinton, IL } \\
050-00461\end{array}$ & III & $\begin{array}{l}\text { BWR-MARK3 } \\
\text { GE } \\
\text { SEL } \\
\text { BALD }\end{array}$ & 2894 & $\begin{array}{c}0930 \\
\text { (MDC) }\end{array}$ & $\begin{array}{l}02 / 24 / 1976 \\
04 / 17 / 1987 \\
11 / 24 / 1987 \\
09 / 29 / 2026\end{array}$ & $\begin{array}{l}\text { OL-FP } \\
\text { NPF-62 }\end{array}$ & \\
\hline $\begin{array}{l}\text { Comanche Peak } 1 \\
\text { Texas Utilities Electric Co. } \\
4 \text { MI N of Glen Rose, TX } \\
050-00445\end{array}$ & IV & $\begin{array}{l}\text { PWR-DRYAMB } \\
\text { WEST 4LP } \\
\text { GEH } \\
\text { BRRT }\end{array}$ & 0000 & $\begin{array}{c}1150 \\
\text { (DER) }\end{array}$ & $12 / 19 / 1974$ & $\begin{array}{l}\text { CP } \\
\text { CPPR-126 }\end{array}$ & $\begin{array}{l}\text { Under Active } \\
\text { Construction }\end{array}$ \\
\hline $\begin{array}{l}\text { Comanche Poak } 2 \\
\text { Texas Utilitios Electric Co. } \\
4 \text { MI N of Glen Rose, TX } \\
050-00446\end{array}$ & IV & $\begin{array}{l}\text { PWR-DRYAMB } \\
\text { WEST ALP } \\
\text { GEH } \\
\text { BRRT }\end{array}$ & 0000 & $\begin{array}{c}1150 \\
\text { (DER) }\end{array}$ & $12 / 19 / 1974$ & $\begin{array}{l}\text { CP } \\
\text { CPPR-127 }\end{array}$ & $\begin{array}{l}\text { Construction } \\
\text { Deferred }\end{array}$ \\
\hline
\end{tabular}




\section{U.S. COMMERCIAL NUCLEAR POWER REACTORS}

\begin{tabular}{|c|c|c|c|c|c|c|c|}
\hline $\begin{array}{c}\text { UNIT } \\
\text { OPERATING UTILITY } \\
\text { LOCATION } \\
\text { DOCKET NUMBER }\end{array}$ & $\begin{array}{c}\text { NRC } \\
\text { REGION }\end{array}$ & $\begin{array}{l}\text { CONTYPE } \\
\text { NSSS } \\
\text { AE } \\
\text { CONST }\end{array}$ & $\begin{array}{l}\text { LIC } \\
\text { MWT }\end{array}$ & $\begin{array}{l}\text { MDC } \\
\text { NET } \\
\text { OR } \\
\text { DER }\end{array}$ & $\begin{array}{l}\text { CP ISSUED } \\
\text { OL ISSUED } \\
\text { COMM. OP } \\
\text { EXP. DATE }\end{array}$ & $\begin{array}{l}\text { LICENSE } \\
\text { TYPE } \\
\text { \& } \\
\text { NUMBER }\end{array}$ & REMARKS \\
\hline $\begin{array}{l}\text { Cook } 1 \\
\text { Indiana \& Michigan Electric Co. } \\
11 \text { MI S of Benton Harbor, MI. } \\
050-00315\end{array}$ & III & $\begin{array}{l}\text { PWR-ICECND } \\
\text { WEST 4LP } \\
\text { AEP } \\
\text { AEP }\end{array}$ & 3250 & $\begin{array}{c}1020 \\
\text { (MDC) }\end{array}$ & $\begin{array}{l}03 / 25 / 1969 \\
10 / 25 / 1974 \\
08 / 28 / 1975 \\
03 / 25 / 2009\end{array}$ & $\begin{array}{l}\text { OL-FP } \\
\text { DPR-58 }\end{array}$ & \\
\hline $\begin{array}{l}\text { Cook } 2 \\
\text { Indiana \& Michigan Power Co. } \\
11 \text { MI S of Benton Harbor, MI } \\
050-00316\end{array}$ & III & $\begin{array}{l}\text { PWR-ICECND } \\
\text { WEST 4LP } \\
\text { AEP }\end{array}$ & 3411 & $\begin{array}{c}1060 \\
\text { (MDC) }\end{array}$ & $\begin{array}{l}03 / 25 / 1969 \\
12 / 23 / 1977 \\
07 / 01 / 1978\end{array}$ & $\begin{array}{l}\text { OL-FP } \\
\text { DPR-74 }\end{array}$ & \\
\hline $\begin{array}{l}\text { Cooper } \\
\text { Nebraska Public Power District } \\
23 \text { MI S of Nebraska City, NE } \\
050-00298\end{array}$ & IV & $\begin{array}{l}\text { BWR-MARK1 } \\
\text { GE } \\
\text { BER } \\
\text { B\&R }\end{array}$ & 2381 & $\begin{array}{c}0764 \\
\text { (MDC) }\end{array}$ & $\begin{array}{l}06 / 04 / 1968 \\
01 / 18 / 1974 \\
07 / 01 / 1974 \\
06 / 04 / 2008\end{array}$ & $\begin{array}{l}\text { OL-FP } \\
\text { DPR-46 }\end{array}$ & \\
\hline $\begin{array}{l}\text { Crystal River } 3 \\
\text { Florida Power Corporation } \\
7 \text { MI NW of Crystal River, FL } \\
050-00302\end{array}$ & II & $\begin{array}{l}\text { PWR-DRYAMB } \\
\text { BEW LLP } \\
\text { GIL } \\
\text { JONES }\end{array}$ & 2544 & $\begin{array}{c}0821 \\
\text { (MDC) }\end{array}$ & $\begin{array}{l}09 / 25 / 1968 \\
01 / 28 / 1977 \\
03 / 13 / 1977 \\
12 / 03 / 2016\end{array}$ & $\begin{array}{l}\text { OL-FP } \\
\text { DPR-72 }\end{array}$ & \\
\hline $\begin{array}{l}\text { Davis-Besse } \\
\text { Toledo Edison Co. } \\
21 \text { MI ESE of Toledo, OH } \\
050-00346\end{array}$ & III & $\begin{array}{l}\text { PWR-DRYAMB } \\
\text { BEW LLP } \\
\text { BECH } \\
\text { BECH }\end{array}$ & $2 \pi / 2$ & $\begin{array}{c}0860 \\
\text { (MDC) }\end{array}$ & $\begin{array}{l}03 / 24 / 1971 \\
04 / 22 / 1977 \\
07 / 31 / 1978 \\
03 / 24 / 2011\end{array}$ & $\begin{array}{l}\text { OL-FP } \\
\text { NPF-3 }\end{array}$ & \\
\hline $\begin{array}{l}\text { Diablo Canyon } 1 \\
\text { Pacific Gas \& Electric Co. } \\
12 \text { MI WSW of San Luis Obispo, CA } \\
050-00275\end{array}$ & v & $\begin{array}{l}\text { PWR-DRYAMB } \\
\text { WEST 4LP } \\
\text { PG\&E } \\
\text { PG\&E }\end{array}$ & 3338 & $\begin{array}{c}1073 \\
\text { (MDC) }\end{array}$ & $\begin{array}{l}04 / 23 / 1968 \\
11 / 02 / 1984 \\
05 / 07 / 1985 \\
04 / 23 / 2008\end{array}$ & $\begin{array}{l}\text { OL-FP } \\
\text { DPR-80 }\end{array}$ & \\
\hline
\end{tabular}




\section{U.S. COMMERCIAL NUCLEAR POWER REACTORS}

\begin{tabular}{|c|c|c|c|c|c|c|c|}
\hline $\begin{array}{c}\text { UNIT } \\
\text { OPERATING UTILITY } \\
\text { LOCATION } \\
\text { DOCKET NUMBER }\end{array}$ & $\begin{array}{c}\text { NRC } \\
\text { REGION }\end{array}$ & $\begin{array}{l}\text { CONTYPE } \\
\text { NSSS } \\
\text { AE } \\
\text { CONST }\end{array}$ & $\begin{array}{l}\text { LIC } \\
\text { MWT }\end{array}$ & $\begin{array}{l}\text { MDC } \\
\text { NET } \\
\text { OR } \\
\text { DER }\end{array}$ & $\begin{array}{l}\text { CP ISSUED } \\
\text { OL ISSUED } \\
\text { COMM. OP } \\
\text { EXP. DATE }\end{array}$ & $\begin{array}{l}\text { LICENSE } \\
\text { TYPE } \\
\text { \& } \\
\text { NUMBER }\end{array}$ & REMARKS \\
\hline $\begin{array}{l}\text { Diablo Canyon } 2 \\
\text { Pacific Gas \& Electric Co. } \\
12 \text { MI WSW of San Luis Obispo } \\
050-00323\end{array}$ & v & $\begin{array}{l}\text { PWR-DRYAMB } \\
\text { WEST ALP } \\
\text { PGEE } \\
\text { PGEE }\end{array}$ & 3411 & $\begin{array}{c}1087 \\
\text { (MDC) }\end{array}$ & $\begin{array}{l}12 / 09 / 1970 \\
08 / 26 / 1985 \\
03 / 13 / 1986 \\
12 / 09 / 2010\end{array}$ & $\begin{array}{l}\text { OL-FP } \\
\text { DPR-82 }\end{array}$ & \\
\hline $\begin{array}{l}\text { Dresden } 2 \\
\text { Commonweaith Edison Co. } \\
9 \text { MI E of Morris, IL } \\
050-00237\end{array}$ & III & $\begin{array}{l}\text { BWR-MARK1 } \\
\text { GE } \\
\text { SEL } \\
\text { UEC }\end{array}$ & 2527 & $\begin{array}{c}0772 \\
(\mathrm{MDC})\end{array}$ & $\begin{array}{l}01 / 10 / 1966 \\
12 / 22 / 1969 \\
06 / 09 / 1970 \\
12 / 22 / 1972\end{array}$ & $\begin{array}{l}\text { OL-P } \\
\text { DPR-19 }\end{array}$ & $\begin{array}{l}\text { License } \\
\text { Not Expired } \\
\text { Under } \\
10 \text { CFR 2.109 }\end{array}$ \\
\hline $\begin{array}{l}\text { Dresden } 3 \\
\text { Commonwealth Edison CO. } \\
9 \text { MI E of Morris, IL } \\
050-00249\end{array}$ & III & $\begin{array}{l}\text { BWR-MARK1 } \\
\text { GE } \\
\text { S\&L } \\
\text { UEC }\end{array}$ & 2527 & $\begin{array}{c}0773 \\
(\mathrm{MDC})\end{array}$ & $\begin{array}{l}10 / 14 / 1966 \\
03 / 02 / 1971 \\
11 / 16 / 1971 \\
10 / 14 / 2006\end{array}$ & $\begin{array}{l}\text { OL-FP } \\
\text { DPR-25 }\end{array}$ & \\
\hline $\begin{array}{l}\text { Duane Arnold } \\
\text { lowa Electric Light \& Power Co. } \\
8 \mathrm{MI} \text { NW of Cedar Rapids, IA } \\
050-00331\end{array}$ & III & $\begin{array}{l}\text { BWR-MARK1 } \\
\text { GE } \quad 4 \\
\text { BECH } \\
\text { BECH }\end{array}$ & 1658 & $\begin{array}{c}0515 \\
\text { (MDC) }\end{array}$ & $\begin{array}{l}06 / 22 / 1970 \\
02 / 22 / 1974 \\
02 / 01 / 1975 \\
06 / 21 / 2010\end{array}$ & $\begin{array}{l}\text { OL-FP } \\
\text { DPR-49 }\end{array}$ & \\
\hline $\begin{array}{l}\text { Farley } 1 \\
\text { Alabama Power Co. } \\
18 \text { MI SE of Dothan, AL } \\
050-00348\end{array}$ & II & $\begin{array}{l}\text { PWR-DRYAMB } \\
\text { WEST } \quad 3 \text { LP } \\
\text { SSI } \\
\text { DANI }\end{array}$ & 2652 & $\begin{array}{c}0813 \\
(\mathrm{MDC})\end{array}$ & $\begin{array}{l}08 / 16 / 1972 \\
06 / 25 / 1977 \\
12 / 01 / 1977 \\
08 / 16 / 2012\end{array}$ & $\begin{array}{l}\text { OL-FP } \\
\text { NPF-2 }\end{array}$ & \\
\hline $\begin{array}{l}\text { Farley } 2 \\
\text { Alabama Power Co. } \\
18 \text { MI SE of Dothan, AL } \\
050-00364\end{array}$ & II & $\begin{array}{l}\text { PWR-DRYAMB } \\
\text { WEST 3LP } \\
\text { SSI } \\
\text { BECH }\end{array}$ & 2652 & $\begin{array}{c}0823 \\
\text { (MDC) }\end{array}$ & $\begin{array}{l}08 / 16 / 1972 \\
03 / 31 / 1981 \\
07 / 30 / 1981 \\
08 / 16 / 2012\end{array}$ & $\begin{array}{l}\text { OL-FP } \\
\text { NPF-8 }\end{array}$ & \\
\hline
\end{tabular}




\section{U.S. COMMERCIAL NUCLEAR POWER REACTORS}

\begin{tabular}{|c|c|c|c|c|c|c|c|}
\hline $\begin{array}{c}\text { UNIT } \\
\text { OPERATING UTILITY } \\
\text { LOCATION } \\
\text { DOCKET NUMBER }\end{array}$ & $\begin{array}{c}\text { NRC } \\
\text { REGION }\end{array}$ & $\begin{array}{l}\text { CONTYPE } \\
\text { NSSS } \\
\text { AE } \\
\text { CONST }\end{array}$ & $\begin{array}{l}\text { LIC } \\
\text { MWT }\end{array}$ & $\begin{array}{l}\text { MDC } \\
\text { NET } \\
\text { OR } \\
\text { DER }\end{array}$ & $\begin{array}{l}\text { CP ISSUED } \\
\text { OL ISSUED } \\
\text { COMM. OP } \\
\text { EXP. DATE }\end{array}$ & $\begin{array}{l}\text { LICENSE } \\
\text { TYPE } \\
\text { \& } \\
\text { NUMBER }\end{array}$ & REMARKS \\
\hline $\begin{array}{l}\text { Fermi } 2 \\
\text { Detroit Edison Co. } \\
25 \text { MI NE of Toledo, OH } \\
050-00341\end{array}$ & III & $\begin{array}{l}\text { BWR-MARK1 } \\
\text { GE } \\
\text { S\&L } \\
\text { DANI }\end{array}$ & 3292 & $\begin{array}{c}1093 \\
\text { (MDC) }\end{array}$ & $\begin{array}{l}09 / 26 / 1972 \\
07 / 15 / 1985 \\
01 / 23 / 1988 \\
03 / 20 / 2025\end{array}$ & $\begin{array}{l}\text { OL-FP } \\
\text { NPF- } 43\end{array}$ & \\
\hline $\begin{array}{l}\text { Fitzpatrick } \\
\text { New York Power Authority } \\
8 \text { MI NE of Oswego, NY } \\
050-00333\end{array}$ & I & $\begin{array}{l}\text { BWR-MARK1 } \\
\text { GE } \\
\text { S\&W } \\
\text { S\&W }\end{array}$ & 2436 & $\begin{array}{c}0778 \\
\text { (MDC) }\end{array}$ & $\begin{array}{l}05 / 20 / 1970 \\
10 / 17 / 1974 \\
07 / 28 / 1975 \\
05 / 20 / 2010\end{array}$ & $\begin{array}{l}\text { OL-FP } \\
\text { DPR-59 }\end{array}$ & \\
\hline $\begin{array}{l}\text { Fort Calhoun } 1 \\
\text { Omaha Public Power District } \\
19 \text { MI N of Omaha, NE } \\
050-00285\end{array}$ & IV & $\begin{array}{l}\text { PWR-DRYAMB } \\
\text { COMB CE } \\
\text { GHDR } \\
\text { GHDR }\end{array}$ & 1500 & $\begin{array}{c}0478 \\
\text { (MDC) }\end{array}$ & $\begin{array}{l}06 / 07 / 1968 \\
08 / 09 / 1973 \\
06 / 20 / 1974 \\
06 / 07 / 2008\end{array}$ & $\begin{array}{l}\text { OL-FP } \\
\text { DPR-40 }\end{array}$ & \\
\hline $\begin{array}{l}\text { Fort St. Vrain } \\
\text { Public Service Co. of Colorado } \\
35 \text { MI N of Denver, CO } \\
050-00267\end{array}$ & IV & $\begin{array}{l}\text { HTGR-GASCLD } \\
\text { GAC } \\
\text { SEL } \\
\text { EBSO }\end{array}$ & 0842 & $\begin{array}{c}0330 \\
(\mathrm{MDC})\end{array}$ & $\begin{array}{l}09 / 17 / 1968 \\
12 / 21 / 1973 \\
07 / 01 / 1979 \\
09 / 17 / 2008\end{array}$ & $\begin{array}{l}\text { OL-FP } \\
\text { DPR-34 }\end{array}$ & \\
\hline $\begin{array}{l}\text { Ginna } \\
\text { Rochester Gas \& Electric Co. } \\
20 \text { MI NE of Rochester, NY } \\
050-00244\end{array}$ & 1 & $\begin{array}{l}\text { PWR-DRYAMB } \\
\text { WEST 2LP } \\
\text { GIL } \\
\text { BECH }\end{array}$ & 1520 & $\begin{array}{c}0470 \\
\text { (MDC) }\end{array}$ & $\begin{array}{l}04 / 25 / 1966 \\
12 / 10 / 1984 \\
07 / 01 / 1970 \\
04 / 25 / 2006\end{array}$ & $\begin{array}{l}\text { OL-FP } \\
\text { DPR-18 }\end{array}$ & \\
\hline $\begin{array}{l}\text { Grand Gulf } 1 \\
\text { System Energy Resources, Inc. } \\
25 \text { MI S of Vicksburg, MS } \\
050-00416\end{array}$ & II & $\begin{array}{l}\text { BWR-MARK3 } \\
\text { GE } \\
\text { BECH } \\
\text { BECH }\end{array}$ & 3833 & $\begin{array}{c}1142 \\
\text { (MDC) }\end{array}$ & $\begin{array}{l}09 / 04 / 1974 \\
11 / 01 / 1984 \\
07 / 01 / 1985 \\
06 / 16 / 2022\end{array}$ & $\begin{array}{l}\text { OL-FP } \\
\text { NPF-29 }\end{array}$ & \\
\hline
\end{tabular}




\section{U.S. COMMERCIAL NUCLEAR POWER REACTORS}

\begin{tabular}{|c|c|c|c|c|c|c|c|}
\hline $\begin{array}{c}\text { UNIT } \\
\text { OPERATING UTILITY } \\
\text { LOCATION } \\
\text { DOCKET NUMBER }\end{array}$ & $\begin{array}{c}\text { NRC } \\
\text { REGION }\end{array}$ & $\begin{array}{l}\text { CONTYPE } \\
\text { NSSS } \\
\text { AE } \\
\text { CONST }\end{array}$ & $\begin{array}{l}\text { LIC } \\
\text { MWT }\end{array}$ & $\begin{array}{l}\text { MDC } \\
\text { NET } \\
\text { OR } \\
\text { DER }\end{array}$ & $\begin{array}{l}\text { CP ISSUED } \\
\text { OL ISSUED } \\
\text { COMM. OP } \\
\text { EXP. DATE }\end{array}$ & $\begin{array}{l}\text { LICENSE } \\
\text { TYPE } \\
\text { \& } \\
\text { NUMBER }\end{array}$ & REMARKS \\
\hline $\begin{array}{l}\text { Grand Gulf } 2 \\
\text { System Energy Resources, Inc. } \\
25 \text { MI S of Vicksburg, MS } \\
050-00417\end{array}$ & II & $\begin{array}{l}\text { BWR-MARK3 } \\
\text { GE } \\
\text { BECH } \\
\text { BECH }\end{array}$ & 0000 & $\begin{array}{l}1250 \\
\text { (DER) }\end{array}$ & 09/04/1974 & $\begin{array}{l}\text { CP } \\
\text { CPPR-119 }\end{array}$ & $\begin{array}{l}\text { Construction } \\
\text { Halted }\end{array}$ \\
\hline $\begin{array}{l}\text { Haddam Neck } \\
\text { Connecticut Yankee Atomic Power } \\
13 \text { MI E of Meriden, CT } \\
050-00213\end{array}$ & I & $\begin{array}{l}\text { PWR-DRYAMB } \\
\text { WEST 4LP } \\
\text { S\&W } \\
\text { S\&W }\end{array}$ & 1825 & $\begin{array}{c}0569 \\
\text { (MDC) }\end{array}$ & $\begin{array}{l}05 / 26 / 1964 \\
12 / 27 / 1974 \\
01 / 01 / 1968 \\
05 / 26 / 2004\end{array}$ & $\begin{array}{l}\text { OL-FP } \\
\text { DPR-61 }\end{array}$ & \\
\hline $\begin{array}{l}\text { Harris } 1 \\
\text { Carolina Power } \& \text { Light Co. } \\
20 \text { MI SW of Raleigh, NC } \\
050-00400\end{array}$ & II & $\begin{array}{l}\text { PWR-DRYAMB } \\
\text { WEST } 3 \text { LP } \\
\text { EBSO } \\
\text { DANI }\end{array}$ & 2775 & $\begin{array}{c}0860 \\
(\mathrm{MDC})\end{array}$ & $\begin{array}{l}01 / 27 / 1978 \\
01 / 12 / 1987 \\
05 / 02 / 1987 \\
10 / 24 / 2026\end{array}$ & $\begin{array}{l}\text { OL-FP } \\
\text { NPF-63 }\end{array}$ & \\
\hline $\begin{array}{l}\text { Hatch } 1 \\
\text { Georgia Power Co. } \\
11 \text { MI N of Baxley, GA } \\
050-00321\end{array}$ & II & $\begin{array}{l}\text { BWR-MARK1 } \\
\text { GE } \\
\text { BECH } \\
\text { GPO }\end{array}$ & 2436 & $\begin{array}{c}0756 \\
\text { (MDC) }\end{array}$ & $\begin{array}{l}09 / 30 / 1969 \\
10 / 13 / 1974 \\
12 / 31 / 1975 \\
09 / 30 / 2009\end{array}$ & $\begin{array}{l}\text { OL-FP } \\
\text { DPR-57 }\end{array}$ & \\
\hline $\begin{array}{l}\text { Hatch } 2 \\
\text { Georgia Power Co. } \\
11 \text { MI N of Baxley, GA } \\
050-00366\end{array}$ & II & $\begin{array}{l}\text { BWR-MARK1 } \\
\text { GE } \\
\text { BECH } \\
\text { GPO }\end{array}$ & 2436 & $\begin{array}{c}0768 \\
\text { (MDC) }\end{array}$ & $\begin{array}{l}12 / 27 / 1972 \\
06 / 13 / 1978 \\
09 / 05 / 1979 \\
12 / 27 / 2012\end{array}$ & $\begin{array}{l}\text { OL-FP } \\
\text { NPF-5 }\end{array}$ & \\
\hline $\begin{array}{l}\text { Hope Creek } 1 \\
\text { Public Service Electric \& Gas } \\
18 \text { MI SE of Wilmington, DE } \\
050-00354\end{array}$ & I & $\begin{array}{l}\text { BWR-MARK1 } \\
\text { GE } \\
\text { BECH } \\
\text { BECH }\end{array}$ & 3293 & $\begin{array}{c}1067 \\
\text { (MDC) }\end{array}$ & $\begin{array}{l}11 / 04 / 1974 \\
07 / 25 / 1986 \\
12 / 20 / 1986 \\
04 / 11 / 2026\end{array}$ & $\begin{array}{l}\text { OL-FP } \\
\text { NPF-57 }\end{array}$ & \\
\hline
\end{tabular}




\section{U.S. COMMERCIAL NUCLEAR POWER REACTORS}

\begin{tabular}{|c|c|c|c|c|c|c|c|}
\hline $\begin{array}{c}\text { UNIT } \\
\text { OPERATING UTILITY } \\
\text { LOCATION } \\
\text { DOCKET NUMBER }\end{array}$ & $\begin{array}{c}\text { NRC } \\
\text { REGION }\end{array}$ & $\begin{array}{l}\text { CONTVPE } \\
\text { NSSS } \\
\text { AE } \\
\text { CONST }\end{array}$ & $\begin{array}{l}\text { LIC } \\
\text { MWT }\end{array}$ & $\begin{array}{l}\text { MDC } \\
\text { NET } \\
\text { OR } \\
\text { DER }\end{array}$ & $\begin{array}{l}\text { CP ISSUED } \\
\text { OL ISSUED } \\
\text { COMM. OP } \\
\text { EXP. DATE }\end{array}$ & $\begin{array}{l}\text { LICENSE } \\
\text { TYPE } \\
\quad 8 \\
\text { NUMBER }\end{array}$ & REMARKS \\
\hline $\begin{array}{l}\text { Indian Point } 2 \\
\text { Consolidated Edison Co. of NY } \\
24 \text { MI N of New York City, NY } \\
050-00247\end{array}$ & I & $\begin{array}{l}\text { PWR-DRYAMB } \\
\text { WEST 4LP } \\
\text { UEC } \\
\text { WDCO }\end{array}$ & 2758 & $\begin{array}{c}0849 \\
\text { (MDC) }\end{array}$ & $\begin{array}{l}10 / 14 / 1966 \\
09 / 28 / 1973 \\
08 / 01 / 1974 \\
09 / 28 / 2013\end{array}$ & $\begin{array}{l}\text { OL-FP } \\
\text { DPR-26 }\end{array}$ & \\
\hline $\begin{array}{l}\text { Indian Point } 3 \\
\text { New York Power Authority } \\
24 \text { MI N of New York City, NY } \\
050-00286\end{array}$ & I & $\begin{array}{l}\text { PWR-DRYAMB } \\
\text { WEST } \quad \text { LLP } \\
\text { UEC } \\
\text { WDCO }\end{array}$ & 3025 & $\begin{array}{c}0965 \\
\text { (MDC) }\end{array}$ & $\begin{array}{l}08 / 13 / 1969 \\
04 / 05 / 1976 \\
08 / 30 / 1976 \\
08 / 13 / 2009\end{array}$ & $\begin{array}{l}\text { OL-FP } \\
\text { DPR-64 }\end{array}$ & \\
\hline $\begin{array}{l}\text { Kewaunee } \\
\text { Wisconsin Public Service Corp. } \\
27 \text { MI E of Green Bay, WI } \\
050-00305\end{array}$ & III & $\begin{array}{l}\text { PWR-DRYAMB } \\
\text { WEST } \quad 2 L P \\
\text { PSE } \\
\text { PSE }\end{array}$ & 1650 & $\begin{array}{c}0503 \\
\text { (MDC) }\end{array}$ & $\begin{array}{l}08 / 06 / 1968 \\
12 / 21 / 1973 \\
06 / 16 / 1974 \\
08 / 06 / 2008\end{array}$ & $\begin{array}{l}\text { OL-FP } \\
\text { DPR-43 }\end{array}$ & \\
\hline $\begin{array}{l}\text { La Salle } 1 \\
\text { Commonwealth Edison Co. } \\
11 \text { Mi SE of Ottawa, IL } \\
050-00373\end{array}$ & III & $\begin{array}{l}\text { BWR-MARK2 } \\
\text { GE } \\
\text { S\&L } \\
\text { CWE }\end{array}$ & 3323 & $\begin{array}{c}1036 \\
\text { (MDC) }\end{array}$ & $\begin{array}{l}09 / 10 / 1973 \\
08 / 13 / 1982 \\
01 / 01 / 1984 \\
05 / 17 / 2022\end{array}$ & $\begin{array}{l}\text { OL-FP } \\
\text { NPF-11 }\end{array}$ & \\
\hline $\begin{array}{l}\text { La Salle } 2 \\
\text { Commonwealth Edison Co. } \\
11 \text { MI SE of Ottawa, IL } \\
050-00374\end{array}$ & III & $\begin{array}{l}\text { BWR-MARK2 } \\
\text { GE } \\
\text { S\&L } \\
\text { CWE }\end{array}$ & 3323 & $\begin{array}{l}1036 \\
\text { (MDC) }\end{array}$ & $\begin{array}{l}09 / 10 / 1973 \\
03 / 23 / 1984 \\
10 / 19 / 1984 \\
12 / 16 / 2023\end{array}$ & $\begin{array}{l}\text { OL-FP } \\
\text { NPF-18 }\end{array}$ & \\
\hline $\begin{array}{l}\text { Limerick } 1 \\
\text { Philadelphia Electric Co. } \\
21 \text { MI NW of Philadelphia, PA } \\
050-00352\end{array}$ & 1 & $\begin{array}{l}\text { BWR-MARK2 } \\
\text { GE } \\
\text { BECH } \\
\text { BECH }\end{array}$ & 3293 & $\begin{array}{c}1055 \\
\text { (MDC) }\end{array}$ & $\begin{array}{l}06 / 19 / 1974 \\
08 / 08 / 1985 \\
02 / 01 / 1986 \\
10 / 26 / 2024\end{array}$ & $\begin{array}{l}\text { OL-FP } \\
\text { NPF-39 }\end{array}$ & \\
\hline
\end{tabular}




\section{U.S. COMMERCIAL NUCLEAR POWER REACTORS}

\begin{tabular}{|c|c|c|c|c|c|c|c|}
\hline $\begin{array}{c}\text { UNIT } \\
\text { OPERATING UTILITY } \\
\text { LOCATION } \\
\text { DOCKET NUMBER }\end{array}$ & $\begin{array}{c}\text { NRC } \\
\text { REGION }\end{array}$ & $\begin{array}{l}\text { CONTYPE } \\
\text { NSSS } \\
\text { AE } \\
\text { CONST }\end{array}$ & $\begin{array}{l}\text { LIC } \\
\text { MWT }\end{array}$ & $\begin{array}{l}\text { MDC } \\
\text { NET } \\
\text { OR } \\
\text { DER }\end{array}$ & $\begin{array}{l}\text { CP ISSUED } \\
\text { OL ISSUED } \\
\text { COMM. OP } \\
\text { EXP. DATE }\end{array}$ & $\begin{array}{l}\text { LICENSE } \\
\text { TYPE } \\
\& \\
\text { NUMBER }\end{array}$ & REMARKS \\
\hline $\begin{array}{l}\text { Limerick } 2 \\
\text { Philadelphia Electric Co. } \\
21 \text { MI NW of Philadelphia, PA } \\
050-00353\end{array}$ & 1 & $\begin{array}{l}\text { BWR-MARK2 } \\
\text { GE } \\
\text { BECH } \\
\text { BECH }\end{array}$ & 0000 & $\begin{array}{l}1065 \\
\text { (DER) }\end{array}$ & $06 / 19 / 1974$ & $\begin{array}{l}\text { CP } \\
\text { CPPR-107 }\end{array}$ & $\begin{array}{l}\text { Under Active } \\
\text { Construction }\end{array}$ \\
\hline $\begin{array}{l}\text { Maine Yankee } \\
\text { Maine Yankee Atomic Power Co. } \\
10 \text { MI N of Bath, ME } \\
050-00309\end{array}$ & I & $\begin{array}{l}\text { PWR-DRYAMB } \\
\text { COMB CE } \\
\text { S\&W } \\
\text { S\&W }\end{array}$ & 2630 & $\begin{array}{l}0810 \\
\text { (MDC) }\end{array}$ & $\begin{array}{l}10 / 21 / 1968 \\
06 / 29 / 1973 \\
12 / 28 / 1972 \\
10 / 21 / 2008\end{array}$ & $\begin{array}{l}\text { OL-FP } \\
\text { DPR-36 }\end{array}$ & \\
\hline $\begin{array}{l}\text { McGuire } 1 \\
\text { Duke Power Co. } \\
17 \text { MI S of Charlotte, NC } \\
060-00369\end{array}$ & II & $\begin{array}{l}\text { PWR-ICECND } \\
\text { WEST 4LP } \\
\text { DUKE } \\
\text { DUKE }\end{array}$ & 3411 & $\begin{array}{c}1129 \\
(\mathrm{MDC})\end{array}$ & $\begin{array}{l}02 / 23 / 1973 \\
07 / 08 / 1981 \\
12 / 01 / 1981 \\
06 / 12 / 2021\end{array}$ & $\begin{array}{l}\text { OL-FP } \\
\text { NPF-9 }\end{array}$ & \\
\hline $\begin{array}{l}\text { McGuire } 2 \\
\text { Duke Power Co. } \\
17 \text { MI S of Charlotte, NC } \\
050-00370\end{array}$ & II & $\begin{array}{l}\text { PWR-ICECND } \\
\text { WEST 4LP } \\
\text { DUKE } \\
\text { DUKE }\end{array}$ & 3411 & $\begin{array}{c}1129 \\
(\mathrm{MDC})\end{array}$ & $\begin{array}{l}02 / 23 / 1973 \\
05 / 27 / 1983 \\
03 / 01 / 1984 \\
03 / 03 / 2023\end{array}$ & $\begin{array}{l}\text { OL-FP } \\
\text { NPF-17 }\end{array}$ & \\
\hline $\begin{array}{l}\text { Millstone } 1 \\
\text { Northeast Nuclear Energy Co. } \\
3.2 \text { MI ENE to New London, CT } \\
050-00245 \text { to }\end{array}$ & I & $\begin{array}{l}\text { BWR-MARK1 } \\
\text { GE } \\
\text { EBSO } \\
\text { EBSO }\end{array}$ & 2011 & $\begin{array}{c}0654 \\
\text { (MDC) }\end{array}$ & $\begin{array}{l}05 / 19 / 1966 \\
10 / 31 / 1986 \\
03 / 01 / 1971 \\
05 / 19 / 2006\end{array}$ & $\begin{array}{l}\text { OL-FP } \\
\text { DPR-21 }\end{array}$ & \\
\hline $\begin{array}{l}\text { Millstone } 2 \\
\text { Northeast Nuclear Energy Co. } \\
3.2 \text { MI ENE to New London, CT } \\
050-00336 \text { to }\end{array}$ & I & $\begin{array}{l}\text { PWR-DRYAMB } \\
\text { COMB CE } \\
\text { BECH } \\
\text { BECH }\end{array}$ & 2700 & $\begin{array}{c}0863 \\
(\mathrm{MDC})\end{array}$ & $\begin{array}{l}12 / 11 / 1970 \\
09 / 30 / 1975 \\
12 / 26 / 1975 \\
07 / 31 / 2015\end{array}$ & $\begin{array}{l}\text { OL-FP } \\
\text { DPR-65 }\end{array}$ & \\
\hline
\end{tabular}




\section{U.S. COMMERCIAL NUCLEAR POWER REACTORS}

\begin{tabular}{|c|c|c|c|c|c|c|c|}
\hline $\begin{array}{c}\text { UNIT } \\
\text { OPERATING UTILITY } \\
\text { LOCATION } \\
\text { DOCKET NUMBER }\end{array}$ & $\begin{array}{c}\text { NRC } \\
\text { REGION }\end{array}$ & $\begin{array}{l}\text { CONTYPE } \\
\text { NSSS } \\
\text { AE } \\
\text { CONST }\end{array}$ & $\begin{array}{l}\text { LIC } \\
\text { MWT }\end{array}$ & $\begin{array}{l}\text { MDC } \\
\text { NET } \\
\text { OR } \\
\text { DER }\end{array}$ & $\begin{array}{l}\text { CP ISSUED } \\
\text { OL ISSUED } \\
\text { COMM. OP } \\
\text { EXP. DATE }\end{array}$ & $\begin{array}{c}\text { LICENSE } \\
\text { TYPE } \\
\text { \& } \\
\text { NUMBER }\end{array}$ & REMARKS \\
\hline $\begin{array}{l}\text { Millstone } 3 \\
\text { Northeast Nuclear Energy Co. } \\
3.2 \text { MI ENE to New London, CT } \\
050-00423 \text { to }\end{array}$ & 1 & $\begin{array}{l}\text { PWR-DRYSUB } \\
\text { WEST 4LP } \\
\text { S\&W } \\
\text { S\&W }\end{array}$ & 3411 & $\begin{array}{c}1142 \\
\text { (MDC) }\end{array}$ & $\begin{array}{l}08 / 09 / 1974 \\
01 / 31 / 1986 \\
04 / 23 / 1986 \\
11 / 25 / 2025\end{array}$ & $\begin{array}{l}\text { OL-FP } \\
\text { NPF-49 }\end{array}$ & \\
\hline $\begin{array}{l}\text { Monticello } \\
\text { Northern States Power Co. } \\
30 \text { MI NW of Minneapolis, MN } \\
050-00263\end{array}$ & III & $\begin{array}{l}\text { BWR-MARK1 } \\
\text { GE } \\
\text { BECH } \\
\text { BECH }\end{array}$ & 1670 & $\begin{array}{c}0536 \\
\text { (MDC) }\end{array}$ & $\begin{array}{l}06 / 19 / 1967 \\
01 / 09 / 1981 \\
06 / 30 / 1971 \\
09 / 08 / 2010\end{array}$ & $\begin{array}{l}\text { OL-FP } \\
\text { DPR-22 }\end{array}$ & \\
\hline $\begin{array}{l}\text { Nine Mile Point } 1 \\
\text { Niagara Mohawk Power Corp. } \\
6 \text { MI NE of Oswego, NY } \\
050-00220\end{array}$ & 1 & $\begin{array}{l}\text { BWR-MARK1 } \\
\text { GE } \\
\text { NIAG } \\
\text { S\&W }\end{array}$ & 1850 & $\begin{array}{c}0610 \\
\text { (MDC) }\end{array}$ & $\begin{array}{l}04 / 12 / 1965 \\
12 / 26 / 1974 \\
12 / 01 / 1969 \\
04 / 11 / 2005\end{array}$ & $\begin{array}{l}\text { OL-FP } \\
\text { DPR-63 }\end{array}$ & \\
\hline $\begin{array}{l}\text { Nine Mile Point } 2 \\
\text { Niagara Mohawk Power Corp. } \\
6 \text { Mi NE of Oswego, NY } \\
050-00410\end{array}$ & 1 & $\begin{array}{l}\text { BWR-MARK2 } \\
\text { GE } \\
\text { S\&W } \\
\text { S\&W }\end{array}$ & 3323 & $\begin{array}{c}1080 \\
\text { (MDC) }\end{array}$ & $\begin{array}{l}06 / 24 / 1974 \\
07 / 02 / 1987 \\
04 / 05 / 1988 \\
10 / 31 / 2026\end{array}$ & $\begin{array}{l}\text { OL-FP } \\
\text { NPF-69 }\end{array}$ & \\
\hline $\begin{array}{l}\text { North Anna } 1 \\
\text { Virginia Power Co. } \\
40 \text { MI NW of Richmond, VA } \\
050-00338\end{array}$ & II & $\begin{array}{l}\text { PWR-DRYSUB } \\
\text { WEST 3LP } \\
\text { S\&W } \\
\text { S\&W }\end{array}$ & 2893 & $\begin{array}{c}0915 \\
\text { (MDC) }\end{array}$ & $\begin{array}{l}02 / 19 / 1971 \\
04 / 01 / 1978 \\
06 / 06 / 1978 \\
04 / 01 / 2018\end{array}$ & $\begin{array}{l}\text { OL-FP } \\
\text { NPF-4 }\end{array}$ & \\
\hline $\begin{array}{l}\text { North Anna } 2 \\
\text { Virginia Power Co. } \\
40 \text { MI NW of Richmond, VA } \\
050-00339\end{array}$ & II & $\begin{array}{l}\text { PWR-DRYSUB } \\
\text { WEST 3LP } \\
\text { S\&W } \\
\text { S\&W }\end{array}$ & 2893 & $\begin{array}{c}0915 \\
\text { (MDC) }\end{array}$ & $\begin{array}{l}02 / 19 / 1971 \\
08 / 21 / 1980 \\
12 / 14 / 1980 \\
08 / 21 / 2020\end{array}$ & $\begin{array}{l}\text { OL-FP } \\
\text { NPF-7 }\end{array}$ & \\
\hline
\end{tabular}




\section{U.S. COMMERCIAL NUCLEAR POWER REACTORS}

\begin{tabular}{|c|c|c|c|c|c|c|c|}
\hline $\begin{array}{c}\text { UNIT } \\
\text { OPERATING UTILITY } \\
\text { LOCATION } \\
\text { DOCKET NUMBER }\end{array}$ & $\begin{array}{c}\text { NRC } \\
\text { REGION }\end{array}$ & $\begin{array}{l}\text { CONTYPE } \\
\text { NSSS } \\
\text { AE } \\
\text { CONST }\end{array}$ & $\begin{array}{l}\text { LIC } \\
\text { MWT }\end{array}$ & $\begin{array}{l}\text { MDC } \\
\text { NET } \\
\text { OR } \\
\text { DER }\end{array}$ & $\begin{array}{l}\text { CP ISSUED } \\
\text { OL ISSUED } \\
\text { COMM. OP } \\
\text { EXP. DATE }\end{array}$ & $\begin{array}{c}\text { LICENSE } \\
\text { TYPE } \\
\& \\
\text { NUMBER }\end{array}$ & REMARKS \\
\hline $\begin{array}{l}\text { Oconee } 1 \\
\text { Duke Power Co. } \\
30 \mathrm{MI} \text { W of Greenville, SC } \\
050-00269\end{array}$ & II & $\begin{array}{l}\text { PWR-DRYAMB } \\
\text { BEW LLP } \\
\text { DBDB } \\
\text { DUKE }\end{array}$ & 2568 & $\begin{array}{c}0846 \\
\text { (MDC) }\end{array}$ & $\begin{array}{l}11 / 06 / 1967 \\
02 / 06 / 1973 \\
07 / 15 / 1973 \\
02 / 06 / 2013\end{array}$ & $\begin{array}{l}\text { OL-FP } \\
\text { DPR-38 }\end{array}$ & \\
\hline $\begin{array}{l}\text { Oconee } 2 \\
\text { Duke Power Co. } \\
30 \mathrm{MI} \mathrm{W} \text { of Greenville, sC } \\
050-00270\end{array}$ & II & $\begin{array}{l}\text { PWR-DRYAMB } \\
\text { B\&W LLP } \\
\text { DBDB } \\
\text { DUKE }\end{array}$ & 2568 & $\begin{array}{c}0846 \\
\text { (MDC) }\end{array}$ & $\begin{array}{l}11 / 06 / 1967 \\
10 / 06 / 1973 \\
09 / 09 / 1974 \\
10 / 06 / 2013\end{array}$ & $\begin{array}{l}\text { OL-FP } \\
\text { DPR-47 }\end{array}$ & \\
\hline $\begin{array}{l}\text { Oconee } 3 \\
\text { Duke Power Co. } \\
30 \mathrm{MI} \mathrm{W} \text { of Greenville, SC } \\
050-00287\end{array}$ & II & $\begin{array}{l}\text { PWR-DRYAMB } \\
\text { B\&W LLP } \\
\text { DBDB } \\
\text { DUKE }\end{array}$ & 2568 & $\begin{array}{c}0846 \\
\text { (MDC) }\end{array}$ & $\begin{array}{l}11 / 06 / 1967 \\
07 / 19 / 1974 \\
12 / 16 / 1974 \\
07 / 19 / 2014\end{array}$ & $\begin{array}{l}\text { OL-FP } \\
\text { DPR-55 }\end{array}$ & \\
\hline $\begin{array}{l}\text { Oyster Creek } \\
\text { GPU Nuclear Corp. } \\
9 \text { MI S of Toms River, NJ } \\
050-00219\end{array}$ & 1 & $\begin{array}{l}\text { BWR-MARK1 } \\
\text { GE } 2 \\
\text { B\&R } \\
\text { B\&R }\end{array}$ & 1930 & $\begin{array}{c}0620 \\
\text { (MDC) }\end{array}$ & $\begin{array}{l}12 / 15 / 1964 \\
08 / 01 / 1969 \\
12 / 01 / 1969 \\
04 / 09 / 1972\end{array}$ & $\begin{array}{l}\text { OL-P } \\
\text { DPR-16 }\end{array}$ & $\begin{array}{l}\text { License } \\
\text { Not Expired } \\
\text { Under } \\
10 \text { CFR } 2.109\end{array}$ \\
\hline $\begin{array}{l}\text { Palisades } \\
\text { Consumers Power Co. } \\
5 \text { MI S of South Haven, MI } \\
050-00255\end{array}$ & III & $\begin{array}{l}\text { PWR-DRYAMB } \\
\text { COMB CE } \\
\text { BECH } \\
\text { BECH }\end{array}$ & 2530 & $\begin{array}{c}0730 \\
\text { (MDC) }\end{array}$ & $\begin{array}{l}03 / 14 / 1967 \\
10 / 16 / 1972 \\
12 / 31 / 1971 \\
03 / 01 / 1974\end{array}$ & $\begin{array}{l}\text { OL-P } \\
\text { DPR-20 }\end{array}$ & $\begin{array}{l}\text { License } \\
\text { Not Expired } \\
\text { Under } \\
10 \text { CFR } 2.109\end{array}$ \\
\hline $\begin{array}{l}\text { Palo Verde } 1 \\
\text { Arizona Public Service Co. } \\
36 \text { MI W of Phoenix, AZ } \\
050-00528\end{array}$ & v & $\begin{array}{l}\text { PWR-DRYAMB } \\
\text { COMB CE } \\
\text { BECH } \\
\text { BCH }\end{array}$ & 3800 & $\begin{array}{c}1221 \\
\text { (MDC) }\end{array}$ & $\begin{array}{l}05 / 25 / 1976 \\
06 / 01 / 1985 \\
01 / 28 / 1986 \\
12 / 31 / 2024\end{array}$ & $\begin{array}{l}\text { OL-FP } \\
\text { NPF-41 }\end{array}$ & \\
\hline
\end{tabular}




\section{U.S. COMMERCIAL NUCLEAR POWER REACTORS}

\begin{tabular}{|c|c|c|c|c|c|c|c|}
\hline $\begin{array}{c}\text { UNIT } \\
\text { OPERATING UTILITY } \\
\text { LOCATION } \\
\text { DOCKET NUMBER }\end{array}$ & $\begin{array}{c}\text { NRC } \\
\text { REGION }\end{array}$ & $\begin{array}{l}\text { CONTYPE } \\
\text { NSSS } \\
\text { AE } \\
\text { CONST }\end{array}$ & $\begin{array}{l}\text { LIC } \\
\text { MWT }\end{array}$ & $\begin{array}{c}\text { MDC } \\
\text { NET } \\
\text { OR } \\
\text { DER }\end{array}$ & $\begin{array}{l}\text { CP ISSUED } \\
\text { OL ISSUED } \\
\text { COMM. OP } \\
\text { EXP. DATE }\end{array}$ & $\begin{array}{l}\text { LICENSE } \\
\text { TYPE } \\
\text { \& } \\
\text { NUMBER }\end{array}$ & REMARKS \\
\hline $\begin{array}{l}\text { Palo Verde } 2 \\
\text { Arizona Public Service Co. } \\
36 \text { MI W of Phoenix, AZ } \\
050-00529\end{array}$ & v & $\begin{array}{l}\text { PWR-DRYAMB } \\
\text { COMB CE80 } \\
\text { BECH } \\
\text { BECH }\end{array}$ & 3800 & $\begin{array}{c}1221 \\
\text { (MDC) }\end{array}$ & $\begin{array}{l}05 / 25 / 1976 \\
04 / 24 / 1986 \\
09 / 19 / 1986 \\
12 / 09 / 2025\end{array}$ & $\begin{array}{l}\text { OL-FP } \\
\text { NPF-51 }\end{array}$ & \\
\hline $\begin{array}{l}\text { Palo Verde } 3 \\
\text { Arizona Public Service Co. } \\
36 \mathrm{MI} \text { W of Phoenix, AZ } \\
050-00530\end{array}$ & v & $\begin{array}{l}\text { PWR-DRYAMB } \\
\text { COMB CE80 } \\
\text { BECH } \\
\text { BECH }\end{array}$ & 3800 & $\begin{array}{c}1221 \\
\text { (MDC) }\end{array}$ & $\begin{array}{l}05 / 25 / 1976 \\
11 / 25 / 1987 \\
01 / 08 / 1988 \\
03 / 25 / 2027\end{array}$ & $\begin{array}{l}\text { OL-FP } \\
\text { NPF-74 }\end{array}$ & \\
\hline $\begin{array}{l}\text { Peach Bottom } 2 \\
\text { Philadelphia Electric Co. } \\
17.9 \text { MI S of Lancaster, PA } \\
050-002 \pi\end{array}$ & 1 & $\begin{array}{l}\text { BWR-MARK1 } \\
\text { GE } \\
\text { BECH } \\
\text { BECH }\end{array}$ & 3293 & $\begin{array}{c}1051 \\
\text { (MDC) }\end{array}$ & $\begin{array}{l}01 / 31 / 1968 \\
12 / 14 / 1973 \\
07 / 05 / 1974 \\
01 / 31 / 2008\end{array}$ & $\begin{array}{l}\text { OL-FP } \\
\text { DPR-44 }\end{array}$ & \\
\hline $\begin{array}{l}\text { Peach Bottom } 3 \\
\text { Philadelphia Electric Co. } \\
17.9 \text { MI S of Lancaster, PA } \\
050-00278\end{array}$ & 1 & $\begin{array}{l}\text { BWR-MARK1 } \\
\text { GE } \\
\text { BECH } \\
\text { BECH }\end{array}$ & 3293 & $\begin{array}{c}1035 \\
\text { (MDC) }\end{array}$ & $\begin{array}{l}01 / 31 / 968 \\
07 / 02 / 1974 \\
12 / 23 / 1974 \\
01 / 31 / 2008\end{array}$ & $\begin{array}{l}\text { OL-FP } \\
\text { DPR-56 }\end{array}$ & \\
\hline $\begin{array}{l}\text { Perry } 1 \\
\text { Cleveland Electric Illumination } \\
7 \mathrm{MI} \text { NE of Painesville, OH } \\
050-00440\end{array}$ & III & $\begin{array}{l}\text { BWR-MARK3 } \\
\text { GE } \\
\text { GIL } \\
\text { KAIS }\end{array}$ & 3579 & $\begin{array}{c}1205 \\
\text { (MDC) }\end{array}$ & $\begin{array}{l}05 / 03 / 1977 \\
11 / 13 / 1986 \\
11 / 18 / 1987 \\
03 / 18 / 2026\end{array}$ & $\begin{array}{l}\text { OL-FP } \\
\text { NPF-58 }\end{array}$ & \\
\hline $\begin{array}{l}\text { Perry } 2 \\
\text { Cleveland Electric Illumination } \\
7 \mathrm{MI} \mathrm{NE} \text { of Painesville, OH } \\
050-00441\end{array}$ & IIII & $\begin{array}{l}\text { BWR-MARK3 } \\
\text { GE } \quad 6 \\
\text { GIL } \\
\text { KAIS }\end{array}$ & 0000 & $\begin{array}{l}1205 \\
\text { (DER) }\end{array}$ & $05 / 03 / 1977$ & $\begin{array}{l}\text { CP } \\
\text { CPPR-149 }\end{array}$ & $\begin{array}{l}\text { Construction } \\
\text { Halted }\end{array}$ \\
\hline
\end{tabular}




\section{U.S. COMMERCIAL NUCLEAR POWER REACTORS}

\begin{tabular}{|c|c|c|c|c|c|c|c|}
\hline $\begin{array}{c}\text { UNIT } \\
\text { OPERATING UTILITY } \\
\text { LOCATION } \\
\text { DOCKET NUMBER }\end{array}$ & $\begin{array}{c}\text { NRC } \\
\text { REGION }\end{array}$ & $\begin{array}{l}\text { CONTYPE } \\
\text { NSSS } \\
\text { AE } \\
\text { CONST }\end{array}$ & $\begin{array}{l}\text { LIC } \\
\text { MWT }\end{array}$ & $\begin{array}{l}\text { MDC } \\
\text { NET } \\
\text { OR } \\
\text { DER }\end{array}$ & $\begin{array}{l}\text { CP ISSUED } \\
\text { OL ISSUED } \\
\text { COMM. OP } \\
\text { EXP. DATE }\end{array}$ & $\begin{array}{l}\text { LICENSE } \\
\text { TYPE } \\
\& \\
\text { NUMBER }\end{array}$ & REMARKS \\
\hline $\begin{array}{l}\text { Pilgrim } 1 \\
\text { Boston Edison Co. } \\
4 \text { MI SE of Plymouth, MA } \\
050-00293\end{array}$ & 1 & $\begin{array}{l}\text { BWR-MARK1 } \\
\text { GE } \\
\text { BECH } \\
\text { BECH }\end{array}$ & 1998 & $\begin{array}{c}0670 \\
\text { (MDC) }\end{array}$ & $\begin{array}{l}08 / 26 / 1968 \\
09 / 15 / 1972 \\
12 / 01 / 1972 \\
08 / 26 / 2008\end{array}$ & $\begin{array}{l}\text { OL-FP } \\
\text { DPR-35 }\end{array}$ & \\
\hline $\begin{array}{l}\text { Point Beach } 1 \\
\text { Wisconsin Electric Power Co. } \\
13 \text { MI NNW of Manitowoc, WI } \\
050-00266\end{array}$ & III & $\begin{array}{l}\text { PWR-DRYAMB } \\
\text { WEST 2LP } \\
\text { BECH } \\
\text { BECH }\end{array}$ & 1518 & $\begin{array}{c}0485 \\
\text { (MDC) }\end{array}$ & $\begin{array}{l}07 / 19 / 1967 \\
10 / 05 / 1970 \\
12 / 21 / 1970 \\
10 / 05 / 2010\end{array}$ & $\begin{array}{l}\text { OL-FP } \\
\text { DPR-24 }\end{array}$ & \\
\hline $\begin{array}{l}\text { Point Beach } 2 \\
\text { Wisconsin Electric Power Co. } \\
13 \text { MI NNW of Manitowoc, WI } \\
050-00301\end{array}$ & III & $\begin{array}{l}\text { PWR-DRYAMB } \\
\text { WEST 2LP } \\
\text { BECH } \\
\text { BECH }\end{array}$ & 1518 & $\begin{array}{c}0485 \\
\text { (MDC) }\end{array}$ & $\begin{array}{l}07 / 25 / 1968 \\
03 / 08 / 1973 \\
10 / 01 / 1972 \\
03 / 08 / 2013\end{array}$ & $\begin{array}{l}\text { OL-FP } \\
\text { DPR-27 }\end{array}$ & \\
\hline $\begin{array}{l}\text { Prairie Island } 1 \\
\text { Northern States Power Co. } \\
28 \text { MI SE of Minneapolis, MN } \\
050-00282\end{array}$ & III & $\begin{array}{l}\text { PWR-DRYAMB } \\
\text { WEST 2LP } \\
\text { FLUR } \\
\text { NSP }\end{array}$ & 1650 & $\begin{array}{c}0503 \\
\text { (MDC) }\end{array}$ & $\begin{array}{l}06 / 25 / 1968 \\
04 / 05 / 1974 \\
12 / 16 / 1973 \\
08 / 09 / 2013\end{array}$ & $\begin{array}{l}\text { OL-FP } \\
\text { DPR-42 }\end{array}$ & \\
\hline $\begin{array}{l}\text { Prairie Island } 2 \\
\text { Northern States Power Co. } \\
28 \text { MI SE of Minneapolis, MN } \\
050-00306\end{array}$ & III & $\begin{array}{l}\text { PWR-DRYAMB } \\
\text { WEST 2LP } \\
\text { FLUR } \\
\text { NSP }\end{array}$ & 1650 & $\begin{array}{c}0503 \\
(\mathrm{MDC})\end{array}$ & $\begin{array}{l}06 / 25 / 1968 \\
10 / 29 / 1974 \\
12 / 21 / 1974 \\
10 / 29 / 2014\end{array}$ & $\begin{array}{l}\text { OL-FP } \\
\text { DPR-60 }\end{array}$ & \\
\hline $\begin{array}{l}\text { Quad Cities } 1 \\
\text { Commonwealth Edison Co. } \\
20 \text { MI NE of Moline, IL } \\
050-00254\end{array}$ & III & $\begin{array}{l}\text { BWR-MARK1 } \\
\text { GE } \\
\text { S\&L } \\
\text { UEC }\end{array}$ & 2511 & $\begin{array}{c}0769 \\
\text { (MDC) }\end{array}$ & $\begin{array}{l}02 / 15 / 1967 \\
12 / 14 / 1972 \\
02 / 18 / 1973 \\
02 / 15 / 2007\end{array}$ & $\begin{array}{l}\text { OL-FP } \\
\text { DPR-29 }\end{array}$ & \\
\hline
\end{tabular}




\section{U.S. COMMERCIAL NUCLEAR POWER REACTORS}

\begin{tabular}{|c|c|c|c|c|c|c|c|}
\hline $\begin{array}{c}\text { UNIT } \\
\text { OPERATING UTILITY } \\
\text { LOCATION } \\
\text { DOCKET NUMBER }\end{array}$ & $\begin{array}{c}\text { NRC } \\
\text { REGION }\end{array}$ & $\begin{array}{l}\text { CONTYPE } \\
\text { NSSS } \\
\text { AE } \\
\text { CONST }\end{array}$ & $\begin{array}{l}\text { LIC } \\
\text { MWT }\end{array}$ & $\begin{array}{l}\text { MDC } \\
\text { NET } \\
\text { OR } \\
\text { DER }\end{array}$ & $\begin{array}{l}\text { CP ISSUED } \\
\text { OL ISSUED } \\
\text { COMM. OP } \\
\text { EXP. DATE }\end{array}$ & $\begin{array}{l}\text { LICENSE } \\
\text { TYPE } \\
\mathbb{8} \\
\text { NUMBER }\end{array}$ & REMARKS \\
\hline $\begin{array}{l}\text { Quad Cities } 2 \\
\text { Commonwealth Edison Co. } \\
20 \text { MI NE of Moline, IL } \\
050-00265\end{array}$ & III & $\begin{array}{l}\text { BWR-MARK1 } \\
\text { GE } \\
\text { S\&L } \\
\text { UEC }\end{array}$ & 2511 & $\begin{array}{c}0769 \\
\text { (MDC) }\end{array}$ & $\begin{array}{l}02 / 15 / 1967 \\
12 / 14 / 1972 \\
03 / 10 / 1973 \\
02 / 15 / 2007\end{array}$ & $\begin{array}{l}\text { OL-FP } \\
\text { DPR-30 }\end{array}$ & \\
\hline $\begin{array}{l}\text { Rancho Seco } \\
\text { Sacramento Mun. Util. District } \\
25 \text { MI SE of Sacramento, CA } \\
050-00312\end{array}$ & v & $\begin{array}{l}\text { PWR-DRYAMB } \\
\text { BEW LLP } \\
\text { BECH } \\
\text { BECH }\end{array}$ & 2772 & $\begin{array}{c}0873 \\
\text { (MDC) }\end{array}$ & $\begin{array}{l}10 / 11 / 1968 \\
08 / 16 / 1974 \\
04 / 17 / 1975 \\
10 / 11 / 2008\end{array}$ & $\begin{array}{l}\text { OL-FP } \\
\text { DPR-54 }\end{array}$ & \\
\hline $\begin{array}{l}\text { River Bend } 1 \\
\text { Gulf States Utilities Co. } \\
24 \text { MI NNW of Baton Rouge, LA } \\
050-00458\end{array}$ & IV & $\begin{array}{l}\text { BWR-MARK3 } \\
\text { GE } \\
\text { S\&W } \\
\text { S\&W }\end{array}$ & 2894 & $\begin{array}{c}0936 \\
\text { (MDC) }\end{array}$ & $\begin{array}{l}03 / 25 / 1977 \\
11 / 20 / 1985 \\
06 / 16 / 1986 \\
08 / 29 / 2025\end{array}$ & $\begin{array}{l}\text { OL-FP } \\
\text { NPF-47 }\end{array}$ & \\
\hline $\begin{array}{l}\text { Robinson } 2 \\
\text { Carolina Power \& Light Co. } \\
26 \text { MI from Florence, SC } \\
050-00261\end{array}$ & II & $\begin{array}{l}\text { PWR-DRYAMB } \\
\text { WEST } \quad 3 L P \\
\text { EBSO } \\
\text { EBSO }\end{array}$ & 2300 & $\begin{array}{l}0665 \\
\text { (MDC) }\end{array}$ & $\begin{array}{l}04 / 13 / 1967 \\
09 / 23 / 1970 \\
03 / 07 / 1971 \\
04 / 13 / 2007\end{array}$ & $\begin{array}{l}\text { OL-FP } \\
\text { DPR-23 }\end{array}$ & \\
\hline $\begin{array}{l}\text { Salem } 1 \\
\text { Public Service Electric \& Gas } \\
18 \text { MI S of Wilmington, DE } \\
050-00272\end{array}$ & I & $\begin{array}{l}\text { PWR-DRYAMB } \\
\text { WEST } \quad 4 L P \\
\text { PUBS } \\
\text { UEC }\end{array}$ & 3411 & $\begin{array}{c}1106 \\
\text { (MDC) }\end{array}$ & $\begin{array}{l}09 / 25 / 1968 \\
12 / 01 / 1976 \\
06 / 30 / 1977 \\
09 / 25 / 2008\end{array}$ & $\begin{array}{l}\text { OL-FP } \\
\text { DPR-70 }\end{array}$ & \\
\hline $\begin{array}{l}\text { Salem } 2 \\
\text { Public Service Electric \& Gas } \\
18 \text { MI S of Wilmington, DE } \\
050-00311\end{array}$ & 1 & $\begin{array}{l}\text { PWR-DRYAMB } \\
\text { WEST 4LP } \\
\text { PUBS } \\
\text { UEC }\end{array}$ & 3411 & $\begin{array}{c}1106 \\
\text { (MDC) }\end{array}$ & $\begin{array}{l}09 / 25 / 1968 \\
05 / 20 / 1981 \\
10 / 13 / 1981 \\
09 / 25 / 2008\end{array}$ & $\begin{array}{l}\text { OL-FP } \\
\text { DPR-75 }\end{array}$ & \\
\hline
\end{tabular}




\section{U.S. COMMERCIAL NUCLEAR POWER REACTORS}

\begin{tabular}{|c|c|c|c|c|c|c|c|}
\hline $\begin{array}{c}\text { UNIT } \\
\text { OPERATING UTILITY } \\
\text { LOCATION } \\
\text { DOCKET NUMBER }\end{array}$ & $\begin{array}{c}\text { NRC } \\
\text { REGION }\end{array}$ & $\begin{array}{l}\text { CONTYPE } \\
\text { NSSS } \\
\text { AE } \\
\text { CONST }\end{array}$ & $\begin{array}{l}\text { LIC } \\
\text { MWT }\end{array}$ & $\begin{array}{l}\text { MDC } \\
\text { NET } \\
\text { OR } \\
\text { DER }\end{array}$ & $\begin{array}{l}\text { CP ISSUED } \\
\text { OL ISSUED } \\
\text { COMM. OP } \\
\text { EXP. DATE }\end{array}$ & $\begin{array}{l}\text { LICENSE } \\
\text { TYPE } \\
\& \\
\text { NUMBER }\end{array}$ & REMARKS \\
\hline $\begin{array}{l}\text { San Onofre } 1 \\
\text { Southern California Edison Co. } \\
4 \text { MI SE of San Clemente, CA } \\
050-00206\end{array}$ & $v$ & $\begin{array}{l}\text { PWR-DRYAMB } \\
\text { WEST 3LP } \\
\text { BECH } \\
\text { BECH }\end{array}$ & 1347 & $\begin{array}{c}0436 \\
\text { (MDC) }\end{array}$ & $\begin{array}{l}03 / 02 / 1964 \\
03 / 27 / 1967 \\
01 / 01 / 1968 \\
09 / 27 / 1972\end{array}$ & $\begin{array}{l}\text { OL-P } \\
\text { DPR-13 }\end{array}$ & $\begin{array}{l}\text { License } \\
\text { Not Expired } \\
\text { Under } \\
10 \text { CFR 2.109 }\end{array}$ \\
\hline $\begin{array}{l}\text { San Onofre } 2 \\
\text { Southern California Edison Co. } \\
4 \mathrm{MI} \text { SE of San Clemente, CA } \\
050-00361\end{array}$ & v & $\begin{array}{l}\text { PWR-DRYAMB } \\
\text { COMB CE } \\
\text { BECH } \\
\text { BECH }\end{array}$ & 3390 & $\begin{array}{c}1070 \\
\text { (MDC) }\end{array}$ & $\begin{array}{l}10 / 18 / 1973 \\
09 / 07 / 1982 \\
08 / 08 / 1983 \\
10 / 18 / 2013\end{array}$ & $\begin{array}{l}\text { OL-FP } \\
\text { NPF-10 }\end{array}$ & \\
\hline $\begin{array}{l}\text { San Onofre } 3 \\
\text { Southern California Edison Co. } \\
4 \text { MI SE of San Clemente, CA } \\
050-00362\end{array}$ & v & $\begin{array}{l}\text { PWR-DRYAMB } \\
\text { COMB CE } \\
\text { BECH } \\
\text { BECH }\end{array}$ & 3390 & $\begin{array}{c}1080 \\
\text { (MDC) }\end{array}$ & $\begin{array}{l}10 / 18 / 1973 \\
09 / 16 / 1983 \\
04 / 01 / 1984 \\
10 / 18 / 2013\end{array}$ & $\begin{array}{l}\text { OL-FP } \\
\text { NPF-15 }\end{array}$ & \\
\hline $\begin{array}{l}\text { Seabrook } 1 \\
\text { Public Service Co. of NH } \\
13 \text { MI S of Portsmouth, NH } \\
050-00443\end{array}$ & 1 & $\begin{array}{l}\text { PWR-DRYAMB } \\
\text { WEST } \quad 4 L P \\
\text { UEC } \\
\text { UEC }\end{array}$ & 0000 & $\begin{array}{c}1198 \\
\text { (DER) }\end{array}$ & $\begin{array}{l}07 / 07 / 1976 \\
10 / 17 / 1986 \\
10 / 17 / 2026\end{array}$ & $\begin{array}{l}\text { OL-FL } \\
\text { NPF-56 }\end{array}$ & \\
\hline $\begin{array}{l}\text { Sequoyah } 1 \\
\text { Tennessee Valley Authority } \\
9.5 \text { MI NE of Chattanooga, TN } \\
050-00327\end{array}$ & II & $\begin{array}{l}\text { PWR-ICECND } \\
\text { WEST 4LP } \\
\text { TVA } \\
\text { TVA }\end{array}$ & 3411 & $\begin{array}{c}1148 \\
\text { (MDC) }\end{array}$ & $\begin{array}{l}05 / 27 / 1970 \\
09 / 17 / 1980 \\
07 / 01 / 1981 \\
05 / 27 / 2010\end{array}$ & $\begin{array}{l}\text { OL-FP } \\
\text { DPR- } \pi\end{array}$ & \\
\hline $\begin{array}{l}\text { Sequoyah } 2 \\
\text { Tennessee Valley Authority } \\
9.5 \text { MI NE of Chattanooga, TN } \\
050-00328\end{array}$ & II & $\begin{array}{l}\text { PWR-ICECND } \\
\text { WEST 4LP } \\
\text { TVA } \\
\text { TVA }\end{array}$ & 3411 & $\begin{array}{c}1148 \\
\text { (MDC) }\end{array}$ & $\begin{array}{l}05 / 27 / 1970 \\
09 / 15 / 1981 \\
06 / 01 / 1982 \\
05 / 27 / 2010\end{array}$ & $\begin{array}{l}\text { OL-FP } \\
\text { DPR-79 }\end{array}$ & \\
\hline
\end{tabular}




\section{U.S. COMMERCIAL NUCLEAR POWER REACTORS}

\begin{tabular}{|c|c|c|c|c|c|c|c|}
\hline $\begin{array}{l}\text { UNIT } \\
\text { OPERATING UTILITY } \\
\text { LOCATION } \\
\text { DOCKET NUMBER }\end{array}$ & $\begin{array}{c}\text { NRC } \\
\text { REGION }\end{array}$ & $\begin{array}{l}\text { CONTYPE } \\
\text { NSSS } \\
\text { AE } \\
\text { CONST }\end{array}$ & $\begin{array}{l}\text { LIC } \\
\text { MWT }\end{array}$ & $\begin{array}{l}\text { MDC } \\
\text { NET } \\
\text { OR } \\
\text { DER }\end{array}$ & $\begin{array}{l}\text { CP ISSUED } \\
\text { OL ISSUED } \\
\text { COMM. OP } \\
\text { EXP. DATE }\end{array}$ & $\begin{array}{l}\text { LICENSE } \\
\text { TYPE } \\
\mathbf{Q} \\
\text { NUMBER }\end{array}$ & REMARKS \\
\hline $\begin{array}{l}\text { Shoreham } \\
\text { Long Island Lighting Co. } \\
7.1 \mathrm{MI} \text { SSW of Brookhaven, NY (LI) } \\
050-00322\end{array}$ & I & $\begin{array}{l}\text { BWR-MARK2 } \\
\text { GE } \\
\text { S\&W } \\
\text { S\&W }\end{array}$ & 0121 & $\begin{array}{c}0820 \\
\text { (DER) }\end{array}$ & $\begin{array}{l}04 / 14 / 1973 \\
07 / 03 / 1985 \\
04 / 13 / 2013\end{array}$ & $\begin{array}{l}\text { OL-LP } \\
\text { NPF-36 }\end{array}$ & \\
\hline $\begin{array}{l}\text { South Texas } 1 \\
\text { Houston Lighting \& Power Co. } \\
12 \text { MI SSW of Bay City, TX } \\
050-00498\end{array}$ & IV & $\begin{array}{l}\text { PWR-DRYAMB } \\
\text { WEST 4LP } \\
\text { BECH } \\
\text { EBSO }\end{array}$ & 3800 & $\begin{array}{c}1250 \\
\text { (MDC) }\end{array}$ & $\begin{array}{l}12 / 22 / 1975 \\
03 / 22 / 1988 \\
08 / 25 / 1988 \\
08 / 20 / 2027\end{array}$ & $\begin{array}{l}\text { OL-FP } \\
\text { NPF-76 }\end{array}$ & \\
\hline $\begin{array}{l}\text { South Texas } 2 \\
\text { Houston Lighting \& Power Co. } \\
12 \text { MI SSW of Bay City, TX } \\
050-00499\end{array}$ & IV & $\begin{array}{l}\text { PWR-DRYAMB } \\
\text { WEST 4LP } \\
\text { BECH } \\
\text { EBSO }\end{array}$ & 0190 & $\begin{array}{l}1250 \\
\text { (DER) }\end{array}$ & $\begin{array}{l}12 / 22 / 1975 \\
12 / 16 / 1988 \\
12 / 16 / 2028\end{array}$ & $\begin{array}{l}\text { OL-LP } \\
\text { NPF-78 }\end{array}$ & \\
\hline $\begin{array}{l}\text { St. Lucie } 1 \\
\text { Florida Power \& Light Co. } \\
12 \text { MI SE of Ft. Pierce, FL } \\
050-00335\end{array}$ & II & $\begin{array}{l}\text { PWR-DRYAMB } \\
\text { COMB CE } \\
\text { EBSO } \\
\text { EBSO }\end{array}$ & 2700 & $\begin{array}{c}0839 \\
\text { (MDC) }\end{array}$ & $\begin{array}{l}07 / 01 / 1970 \\
03 / 01 / 1976 \\
12 / 21 / 1976 \\
03 / 01 / 2016\end{array}$ & $\begin{array}{l}\text { OL-FP } \\
\text { DPR-67 }\end{array}$ & \\
\hline $\begin{array}{l}\text { St. Lucie } 2 \\
\text { Florida Power \& Light Co. } \\
12 \text { MI SE of Ft. Pierce, FL } \\
050-00389\end{array}$ & II & $\begin{array}{l}\text { PWR-DRYAMB } \\
\text { COMB CE } \\
\text { EBSO } \\
\text { EBSO }\end{array}$ & 2700 & $\begin{array}{c}0839 \\
\text { (MDC) }\end{array}$ & $\begin{array}{l}05 / 02 / 1977 \\
06 / 10 / 1983 \\
08 / 08 / 1983 \\
04 / 06 / 2023\end{array}$ & $\begin{array}{l}\text { OL-FP } \\
\text { NPF-16 }\end{array}$ & \\
\hline $\begin{array}{l}\text { Summer } \\
\text { South Carolina Electric \& Gas } \\
26 \text { MI NW of Columbia, SC } \\
050-00395\end{array}$ & II & $\begin{array}{l}\text { PWR-DRYAMB } \\
\text { WEST } \quad 3 L P \\
\text { GIL } \\
\text { DANI }\end{array}$ & 2775 & $\begin{array}{c}0885 \\
\text { (MDC) }\end{array}$ & $\begin{array}{l}03 / 21 / 1973 \\
11 / 12 / 1982 \\
01 / 01 / 1984 \\
03 / 21 / 2023\end{array}$ & $\begin{array}{l}\text { OL-FP } \\
\text { NPF-12 }\end{array}$ & \\
\hline
\end{tabular}




\section{U.S. COMMERCIAL NUCLEAR POWER REACTORS}

\begin{tabular}{|c|c|c|c|c|c|c|c|}
\hline $\begin{array}{c}\text { UNIT } \\
\text { OPERATING UTILITY } \\
\text { LOCATION } \\
\text { DOCKET NUMBER }\end{array}$ & $\begin{array}{c}\text { NRC } \\
\text { REGION }\end{array}$ & $\begin{array}{l}\text { CONTYPE } \\
\text { NSSS } \\
\text { AE } \\
\text { CONST }\end{array}$ & $\begin{array}{l}\text { LIC } \\
\text { MWT }\end{array}$ & $\begin{array}{l}\text { MDC } \\
\text { NET } \\
\text { OR } \\
\text { DER }\end{array}$ & $\begin{array}{l}\text { CP ISSUED } \\
\text { OL ISSUED } \\
\text { COMM. OP } \\
\text { EXP. DATE }\end{array}$ & $\begin{array}{l}\text { LICENSE } \\
\text { TYPE } \\
8 \\
\text { NUMBER }\end{array}$ & REMARKS \\
\hline $\begin{array}{l}\text { Surry } 1 \\
\text { Virginia Power Co. } \\
17 \text { MI NW of Newport News, VA } \\
050-00280\end{array}$ & II & $\begin{array}{l}\text { PWR-DRYSUB } \\
\text { WEST 3LP } \\
\text { S\&W } \\
\text { S\&W }\end{array}$ & 2441 & $\begin{array}{c}0781 \\
\text { (MDC) }\end{array}$ & $\begin{array}{l}06 / 25 / 1968 \\
05 / 25 / 1972 \\
12 / 22 / 1972 \\
05 / 25 / 2012\end{array}$ & $\begin{array}{l}\text { OL-FP } \\
\text { DPR-32 }\end{array}$ & \\
\hline $\begin{array}{l}\text { Surry } 2 \\
\text { Virginia Power Co. } \\
17 \text { MI NW of Newport News, VA } \\
050-00281\end{array}$ & II & $\begin{array}{l}\text { PWR-DRYSUB } \\
\text { WEST 3LP } \\
\text { S\&W } \\
\text { S\&W }\end{array}$ & 2441 & $\begin{array}{c}0781 \\
\text { (MDC) }\end{array}$ & $\begin{array}{l}06 / 25 / 1968 \\
01 / 29 / 1973 \\
05 / 01 / 1973 \\
01 / 29 / 2013\end{array}$ & $\begin{array}{l}\text { OL-FP } \\
\text { DPR-37 }\end{array}$ & \\
\hline $\begin{array}{l}\text { Susquehanna } 1 \\
\text { Pennsylvania Power \& Light Co. } \\
7 \text { MI NE of Berwick, PA } \\
\text { 050-00387 }\end{array}$ & 1 & $\begin{array}{l}\text { BWR-MARK2 } \\
\text { GE } \\
\text { BECH } \\
\text { BECH }\end{array}$ & 3293 & $\begin{array}{c}1032 \\
\text { (MDC) }\end{array}$ & $\begin{array}{l}11 / 02 / 1973 \\
11 / 12 / 1982 \\
06 / 08 / 1983 \\
07 / 17 / 2022\end{array}$ & $\begin{array}{l}\text { OL-FP } \\
\text { NPF-14 }\end{array}$ & \\
\hline $\begin{array}{l}\text { Susquehanna } 2 \\
\text { Pennsylvania Power ( Light Co. } \\
7 \text { MI NE of Berwick, PA } \\
050-00388\end{array}$ & I & $\begin{array}{l}\text { BWR-MARK2 } \\
\text { GE } \\
\text { BECH } \\
\text { BECH }\end{array}$ & 3293 & $\begin{array}{c}1032 \\
\text { (MDC) }\end{array}$ & $\begin{array}{l}11 / 02 / 1973 \\
06 / 27 / 1984 \\
02 / 12 / 1985 \\
03 / 23 / 2024\end{array}$ & $\begin{array}{l}\text { OL-FP } \\
\text { NPF-22 }\end{array}$ & \\
\hline $\begin{array}{l}\text { Three Mile Island 1 } \\
\text { GPU Nuclear Corp. } \\
10 \text { MI SE of Harrisburg, PA } \\
050-00289\end{array}$ & 1 & $\begin{array}{l}\text { PWR-DRYAMB } \\
\text { B\&W LLP } \\
\text { GIL } \\
\text { UEC }\end{array}$ & 2535 & $\begin{array}{c}0776 \\
\text { (MDC) }\end{array}$ & $\begin{array}{l}05 / 18 / 1968 \\
04 / 19 / 1974 \\
09 / 02 / 1974 \\
05 / 18 / 2008\end{array}$ & $\begin{array}{l}\text { OL-FP } \\
\text { DPR-50 }\end{array}$ & \\
\hline $\begin{array}{l}\text { Trojan } \\
\text { Portland General Electric Co. } \\
32 \text { MI N of Portland, OR } \\
050-00344\end{array}$ & v & $\begin{array}{l}\text { PWR-DRYAMB } \\
\text { WEST } \quad 4 \text { LP } \\
\text { BECH } \\
\text { BECH }\end{array}$ & 3411 & $\begin{array}{c}1095 \\
\text { (MDC) }\end{array}$ & $\begin{array}{l}02 / 08 / 1971 \\
11 / 21 / 1975 \\
05 / 20 / 1976 \\
02 / 08 / 2011\end{array}$ & $\begin{array}{l}\text { OL-FP } \\
\text { NPF-1 }\end{array}$ & \\
\hline
\end{tabular}




\section{U.S. COMMERCIAL NUCLEAR POWER REACTORS}

\begin{tabular}{|c|c|c|c|c|c|c|c|}
\hline $\begin{array}{c}\text { UNIT } \\
\text { OPERATING UTILITY } \\
\text { LOCATION } \\
\text { DOCKET NUMBER }\end{array}$ & $\begin{array}{c}\text { NRC } \\
\text { REGION }\end{array}$ & $\begin{array}{l}\text { CONTYPE } \\
\text { NSSS } \\
\text { AE } \\
\text { CONST }\end{array}$ & $\begin{array}{l}\text { LIC } \\
\text { MWT }\end{array}$ & $\begin{array}{l}\text { MDC } \\
\text { NET } \\
\text { OR } \\
\text { DER }\end{array}$ & $\begin{array}{l}\text { CP ISSUED } \\
\text { OL ISSUED } \\
\text { COMM. OP } \\
\text { EXP. DATE }\end{array}$ & $\begin{array}{l}\text { LICENSE } \\
\text { TYPE } \\
\text { \& } \\
\text { NUMBER }\end{array}$ & REMARKS \\
\hline $\begin{array}{l}\text { Turkey Point } 3 \\
\text { Florida Power \& Light Co. } \\
25 \text { Mi S of Miami, FL } \\
050-00250\end{array}$ & II & $\begin{array}{l}\text { PWR-DRYAMB } \\
\text { WEST } \quad 3 \text { PP } \\
\text { BECH } \\
\text { BECH }\end{array}$ & 2200 & $\begin{array}{c}0666 \\
\text { (MDC) }\end{array}$ & $\begin{array}{l}04 / 27 / 1967 \\
07 / 19 / 1972 \\
12 / 14 / 1972 \\
04 / 27 / 2007\end{array}$ & $\begin{array}{l}\text { OL-FP } \\
\text { DPR-31 }\end{array}$ & \\
\hline $\begin{array}{l}\text { Turkey Point } 4 \\
\text { Florida Power \& Light Co. } \\
25 \text { MI S of Miaml, FL } \\
050-00251\end{array}$ & II & $\begin{array}{l}\text { PWR-DRYAMB } \\
\text { WEST 3LP } \\
\text { BECH } \\
\text { BECH }\end{array}$ & 2200 & $\begin{array}{c}0666 \\
\text { (MDC) }\end{array}$ & $\begin{array}{l}04 / 27 / 1967 \\
04 / 10 / 1973 \\
09 / 07 / 1973 \\
04 / 27 / 2007\end{array}$ & $\begin{array}{l}\text { OL-FP } \\
\text { DPR-41 }\end{array}$ & \\
\hline $\begin{array}{l}\text { Vermont Yankee } \\
\text { Vermont Yankee Nuclear Power Co. } \\
5 \text { MI S of Brattleboro, VT } \\
050-00271\end{array}$ & 1 & $\begin{array}{l}\text { BWR-MARK1 } \\
\text { GE } \\
\text { EBSO } \\
\text { EBSO }\end{array}$ & 1593 & $\begin{array}{c}0504 \\
\text { (MDC) }\end{array}$ & $\begin{array}{l}12 / 11 / 1967 \\
02 / 28 / 1973 \\
11 / 30 / 1972 \\
12 / 11 / 2007\end{array}$ & $\begin{array}{l}\text { OL-FP } \\
\text { DPR-28 }\end{array}$ & \\
\hline $\begin{array}{l}\text { Vogtle } 1 \\
\text { Georgia Power Co. } \\
26 \text { MI SE of Augusta, GA } \\
050-00424\end{array}$ & II & $\begin{array}{l}\text { PWR-DRYAMB } \\
\text { WEST } \quad 4 L P \\
\text { SBEC } \\
\text { GPO }\end{array}$ & 3411 & $\begin{array}{c}1079 \\
\text { (MDC) }\end{array}$ & $\begin{array}{l}06 / 28 / 1974 \\
03 / 16 / 1987 \\
06 / 01 / 1987 \\
01 / 16 / 2027\end{array}$ & $\begin{array}{l}\text { OL-FP } \\
\text { NPF-68 }\end{array}$ & \\
\hline $\begin{array}{l}\text { Vogtle } 2 \\
\text { Georgia Power Co. } \\
26 \text { MI SE of Augusta, GA } \\
050-00425\end{array}$ & II & $\begin{array}{l}\text { PWR-DRYAMB } \\
\text { WEST } \quad 4 L P \\
\text { SBEC } \\
\text { GPO }\end{array}$ & 0000 & $\begin{array}{l}1101 \\
\text { (DER) }\end{array}$ & 06/28/1974 & $\begin{array}{l}\text { CP } \\
\text { CPPR-109 }\end{array}$ & $\begin{array}{l}\text { Under Active } \\
\text { Construction }\end{array}$ \\
\hline $\begin{array}{l}\text { Washington Nuclear } 1 \\
\text { Washington Public Power Supply } \\
12 \text { MI NW of Richland, WA } \\
050-00460\end{array}$ & v & $\begin{array}{l}\text { PWR-DRYAMB } \\
\text { B\&W LLP } \\
\text { UEC } \\
\text { BECH }\end{array}$ & 0000 & $\begin{array}{l}1266 \\
\text { (DER) }\end{array}$ & $12 / 24 / 1975$ & $\begin{array}{l}\text { CP } \\
\text { CPPR-134 }\end{array}$ & $\begin{array}{l}\text { Construction } \\
\text { Halted }\end{array}$ \\
\hline
\end{tabular}




\section{U.S. COMMERCIAL NUCLEAR POWER REACTORS}

\begin{tabular}{|c|c|c|c|c|c|c|c|}
\hline $\begin{array}{c}\text { UNIT } \\
\text { OPERATING UTILITY } \\
\text { LOCATION } \\
\text { DOCKET NUMBER }\end{array}$ & $\begin{array}{c}\text { NRC } \\
\text { REGION }\end{array}$ & $\begin{array}{l}\text { CONTYPE } \\
\text { NSSS } \\
\text { AE } \\
\text { CONST }\end{array}$ & $\begin{array}{l}\text { LIC } \\
\text { MWT }\end{array}$ & $\begin{array}{l}\text { MDC } \\
\text { NET } \\
\text { OR } \\
\text { DER }\end{array}$ & $\begin{array}{l}\text { CP ISSUED } \\
\text { OL ISSUED } \\
\text { COMM. OP } \\
\text { EXP. DATE }\end{array}$ & $\begin{array}{l}\text { LICENSE } \\
\text { TYPE } \\
8 \\
\text { NUMBER }\end{array}$ & REMARKS \\
\hline $\begin{array}{l}\text { Washington Nuclear } 2 \\
\text { Washington Public Power Supply } \\
12 \text { MI NW of Richland, WA } \\
050-00397\end{array}$ & v & $\begin{array}{l}\text { BWR-MARK2 } \\
\text { GE } \\
\text { B\&R } \\
\text { BECH }\end{array}$ & 3323 & $\begin{array}{c}1095 \\
(\mathrm{MDC})\end{array}$ & $\begin{array}{l}03 / 19 / 1973 \\
04 / 13 / 1984 \\
12 / 13 / 1984 \\
12 / 20 / 2023\end{array}$ & $\begin{array}{l}\text { OL-FP } \\
\text { NPF-21 }\end{array}$ & \\
\hline $\begin{array}{l}\text { Washington Nuclear } 3 \\
\text { Washington Pubiic Power Supply } \\
26 \mathrm{MI} \text { W of Olympia, WA } \\
050-00508\end{array}$ & v & $\begin{array}{l}\text { PWR-DRYAMB } \\
\text { COMB CE80 } \\
\text { EBSO } \\
\text { EBSO }\end{array}$ & 0000 & $\begin{array}{c}1242 \\
\text { (DER) }\end{array}$ & 04/11/1978 & $\begin{array}{l}\text { CP } \\
\text { CPPR-154 }\end{array}$ & $\begin{array}{l}\text { Construction } \\
\text { Halted }\end{array}$ \\
\hline $\begin{array}{l}\text { Waterford } 3 \\
\text { Louisiana Power \& Light Co. } \\
20 \text { MI W of New Orleans, LA } \\
050-00382\end{array}$ & IV & $\begin{array}{l}\text { PWR-DRYAMB } \\
\text { COMB CE } \\
\text { EBSO } \\
\text { EBSO }\end{array}$ & 3390 & $\begin{array}{c}1075 \\
\text { (MDC) }\end{array}$ & $\begin{array}{l}11 / 14 / 1974 \\
03 / 16 / 1985 \\
09 / 24 / 1985 \\
12 / 18 / 2024\end{array}$ & $\begin{array}{l}\text { OL-FP } \\
\text { NPF-38 }\end{array}$ & \\
\hline $\begin{array}{l}\text { Watts Bar } 1 \\
\text { Tennessee Valley Authority } \\
10 \text { MI S of Spring City, TN } \\
050-00390\end{array}$ & II & $\begin{array}{l}\text { PWR-ICECND } \\
\text { WEST 4LP } \\
\text { TVA } \\
\text { TVA }\end{array}$ & 0000 & $\begin{array}{l}1165 \\
\text { (DER) }\end{array}$ & $01 / 23 / 1973$ & $\begin{array}{l}\text { CP } \\
\text { CPPR-91 }\end{array}$ & $\begin{array}{l}\text { Under Active } \\
\text { Construction }\end{array}$ \\
\hline $\begin{array}{l}\text { Watts Bar } 2 \\
\text { Tennessee Valley Authority } \\
10 \text { MI S of Spring City, TN } \\
050-00391\end{array}$ & II & $\begin{array}{l}\text { PWR-ICECND } \\
\text { WEST 4LP } \\
\text { TVA } \\
\text { TVA }\end{array}$ & 0000 & $\begin{array}{l}1165 \\
\text { (DER) }\end{array}$ & $01 / 23 / 1973$ & $\begin{array}{l}\text { CP } \\
\text { CPPR-92 }\end{array}$ & $\begin{array}{l}\text { Under Active } \\
\text { Construction }\end{array}$ \\
\hline $\begin{array}{l}\text { Wolf Creek } 1 \\
\text { Wolf Creek Nuclear Operating Co. } \\
3.5 \text { MI NE of Burlington, KS } \\
050-00482\end{array}$ & IV & $\begin{array}{l}\text { PWR-DRYAMB } \\
\text { WEST 4LP } \\
\text { BECH } \\
\text { DANI }\end{array}$ & 3411 & $\begin{array}{c}1128 \\
\text { (MDC) }\end{array}$ & $\begin{array}{l}05 / 31 / 1977 \\
06 / 04 / 1985 \\
09 / 03 / 1985 \\
03 / 11 / 2025\end{array}$ & $\begin{array}{l}\text { OL-FP } \\
\text { NPF-42 }\end{array}$ & \\
\hline
\end{tabular}




\section{U.S. COMMERCIAL NUCLEAR POWER REACTORS}

\begin{tabular}{|c|c|c|c|c|c|c|c|}
\hline $\begin{array}{c}\text { UNIT } \\
\text { OPERATING UTILITY } \\
\text { LOCATION } \\
\text { DOCKET NUMBER }\end{array}$ & $\begin{array}{c}\text { NRC } \\
\text { REGION }\end{array}$ & $\begin{array}{l}\text { CONTYPE } \\
\text { NSSS } \\
\text { AE } \\
\text { CONST }\end{array}$ & $\begin{array}{l}\text { LIC } \\
\text { MWT }\end{array}$ & $\begin{array}{l}\text { MDC } \\
\text { NET } \\
\text { OR } \\
\text { DER }\end{array}$ & $\begin{array}{l}\text { CP ISSUED } \\
\text { OL ISSUED } \\
\text { COMM. OP } \\
\text { EXP. DATE }\end{array}$ & $\begin{array}{l}\text { LICENSE } \\
\text { TYPE } \\
8 \\
\text { NUMBER }\end{array}$ & REMARKS \\
\hline $\begin{array}{l}\text { Yankee-Rowe } \\
\text { Yankee Atomic Electric Co. } \\
21 \text { MI NE of Pittsfield, MA } \\
050-00029\end{array}$ & I & $\begin{array}{l}\text { PWR-DRYAMB } \\
\text { WEST 4LP } \\
\text { S\&W } \\
\text { S\&W }\end{array}$ & 0600 & $\begin{array}{c}0167 \\
\text { (MDC) }\end{array}$ & $\begin{array}{l}11 / 04 / 1957 \\
12 / 24 / 1963 \\
07 / 01 / 1961 \\
07 / 09 / 2000\end{array}$ & $\begin{array}{l}\text { OL-FP } \\
\text { DPR-3 }\end{array}$ & \\
\hline $\begin{array}{l}\text { Zion } 1 \\
\text { Commonwealth Edison Co. } \\
40 \mathrm{MI} N \text { of Chicago, IL } \\
050-00295\end{array}$ & III & $\begin{array}{l}\text { PWR-DRYAMB } \\
\text { WEST 4LP } \\
\text { S\&L } \\
\text { CWE }\end{array}$ & 3250 & $\begin{array}{c}1040 \\
\text { (MDC) }\end{array}$ & $\begin{array}{l}12 / 26 / 1968 \\
10 / 19 / 1973 \\
12 / 31 / 1973 \\
12 / 26 / 2008\end{array}$ & $\begin{array}{l}\text { OL-FP } \\
\text { DPR-39 }\end{array}$ & \\
\hline $\begin{array}{l}\text { Zion } 2 \\
\text { Commonwealth Edison Co. } \\
40 \mathrm{MI} \mathrm{N} \text { of Chicago, IL } \\
050-00304\end{array}$ & III & $\begin{array}{l}\text { PWR-DRYAMB } \\
\text { WEST 4LP } \\
\text { S\&L } \\
\text { CWE }\end{array}$ & 3250 & $\begin{array}{c}1040 \\
\text { (MDC) }\end{array}$ & $\begin{array}{l}12 / 26 / 1968 \\
11 / 14 / 1973 \\
09 / 17 / 1974 \\
12 / 26 / 2008\end{array}$ & $\begin{array}{l}\text { OL-FP } \\
\text { DPR-48 }\end{array}$ & \\
\hline
\end{tabular}




\section{COMMERCIAL NUCLEAR POWER PLANTS FORMERLY LICENSED TO OPERATE}

\begin{tabular}{|l|c|c|c|}
\hline \multicolumn{1}{|c|}{ UNIT NAME } & $\begin{array}{c}\text { INITIAL OPERATING } \\
\text { LICENSE DATE }\end{array}$ & $\begin{array}{c}\text { FIRST ELECTRICAL } \\
\text { GENERATION DATE }\end{array}$ & $\begin{array}{c}\text { SHUTDOWN } \\
\text { DATE }\end{array}$ \\
\hline DRESDEN 1 & $09 / 28 / 59$ & $04 / 15 / 60$ & $10 / 31 / 78$ \\
HALLAM & $01 / 02 / 62$ & $05 / 29 / 63$ & $09 / 01 / 64$ \\
INDIAN POINT 1 & $03 / 26 / 62$ & $09 / 16 / 62$ & $10 / 31 / 74$ \\
PIQUA & $08 / 23 / 62$ & $11 / 04 / 63$ & $01 / 01 / 66$ \\
HUMBOLDT BAY & $08 / 28 / 62$ & $04 / 18 / 63$ & $07 / 02 / 76$ \\
ELK RIVER & $11 / 06 / 62$ & $08 / 24 / 63$ & $02 / 01 / 68$ \\
CVTR & $11 / 27 / 62$ & $12 / 18 / 63$ & $01 / 01 / 67$ \\
FERMI 1 & $05 / 10 / 63$ & $08 / 05 / 66$ & $11 / 29 / 72$ \\
PATHFINDER & $03 / 12 / 64$ & $07 / 25 / 66$ & $10 / 01 / 67$ \\
BONUS & $04 / 02 / 64$ & $08 / 14 / 64$ & $06 / 01 / 68$ \\
PEACH BOTTOM 1 & $01 / 24 / 66$ & $01 / 27 / 67$ & $11 / 01 / 74$ \\
LA CROSSE & $07 / 03 / 67$ & $04 / 26 / 68$ & $04 / 30 / 87$ \\
THREE MILE ISLAND 2 & $02 / 08 / 78$ & $04 / 21 / 78$ & $03 / 28 / 79$ \\
\hline
\end{tabular}


REACTOR CANCELLATIONS*

UNITS UNDER CONSTRUCTION PERMIT REVIEW

\begin{tabular}{|c|c|c|c|c|}
\hline PLANT & UTILITY & TYPE & $\begin{array}{l}\text { MWE/PER } \\
\text { UNIT }\end{array}$ & DATE \\
\hline TYRONE 2 & NORTHERN STATES POWER & PWR & 1250 & FY-75 \\
\hline QUANICASSEE $1 \& 2$ & CONSUMERS POWER & PWR & 1150 & FY-75 \\
\hline PILGRIM 3 & BOSTON EDISON & PWR & 1180 & FY-75 \\
\hline BARTON $3 \& 4$ & ALABAMA POWER \& LIGHT & BWR & 1159 & FY-67 \\
\hline SUMMIT 2 & DELMARVA POWER \& LIGHT & HTGR & 1200 & FY-T \\
\hline ALLENS CREEK 2 & HOUSTON LIGHTING AND POWER COMPANY & BWR & 1213 & FY-TI \\
\hline BLUE HILLS 1 \& 2 & GULF STATES UTILITIES & PWR & 918 & FY-78 \\
\hline SUMMIT 1 & DELMARVA POWER AND LIGHT & HTGR & 1200 & FY-78 \\
\hline SUNDESERT $1 \& 2$ & SAN DIEGO GAS AND ELECTRIC & PWR & 974 & FY-78 \\
\hline DOUGLAS POINT 2 & POTOMAC ELECTRIC POWER & BWR & 1146 & FY.78 \\
\hline BARTON 1 \& 2 & ALABAMA POWER \& LIGHT & BWR & 1159 & FY-78 \\
\hline FORT CALHOUN 2 & OMAHA PUBLIC POWER DISTRICT & PWR & 1136 & FY78 \\
\hline $\begin{array}{l}\text { HAVEN } 2 \\
\text { (formerly KOSHKONONG 2) }\end{array}$ & WISCONSIN ELECTRIC POWER CO. & PWR & 900 & FY-79 \\
\hline ATLANTIC $1 \& 2$ & PUBLIC SERVICE ELECTRIC AND GAS & PWR & 1150 & FY-79 \\
\hline GREENE COUNTY & POWER AUTHORITY OF STATE OF NEW YORK & PWR & 1191 & FY-79 \\
\hline PALO VERDE 4 \& 5 & ARIZONA PUBLIC SERVICE & PWR & 1270 & FY-79 \\
\hline DOUGLAS POINT 1 & POTOMAC ELECTRIC POWER & BWR & 1146 & FY-79 \\
\hline FULTON $1 \& 2$ & PHILADELPHIA ELECTRIC & HTGR & 1160 & FY-79 \\
\hline NORTH COAST 2 & PUERTO RICO WATER RESOURCES AUTHORITY & PWR & 583 & FY-79 \\
\hline ERIE 182 & OHIO EDISON CO. & PWR & 1260 & FY-80 \\
\hline DAVIS-BESSE $2 \& 3$ & TOLEDO EDISON CO. & PWR & 906 & FY-80 \\
\hline NEW ENGLAND $1 \& 2$ & NEW ENGLAND POWER & PWR & 1194 & FY-80 \\
\hline
\end{tabular}




\section{REACTOR CANCELLATIONS* UNITS UNDER CONSTRUCTION PERMIT REVIEW}

\begin{tabular}{|l|l|l|c|c|}
\hline \multicolumn{1}{|c|}{ PLANT } & \multicolumn{1}{|c|}{ UTILITY } & TYPE & $\begin{array}{c}\text { MWE/PER } \\
\text { UNIT }\end{array}$ & DATE \\
\hline $\begin{array}{l}\text { HAVEN 1 } \\
\text { (fOrmerly KOSHKONONG 1) }\end{array}$ & WISCONSIN ELECTRIC POWER CO. & PWR & 900 & FY-80 \\
GREENWOOD 2 \&3 & DETROIT EDISON & PWR & 1200 & FY-80 \\
MONTAGUE 1 \& 2 & NORTHEAST NUCLEAR ENERGY CO. & BWR & 1150 & FY-81 \\
NEW HAVEN 182 & NEW YORK STATE ELECTRIC \& GAS & PWR & 1250 & FY-81 \\
PILGRIM 2 & POSTON EDISON & 1180 & FY-81 \\
PERKINS 1, 8 \& 3 & DUKE POWER & PWR & 1280 & FY-82 \\
BLACK FOX 1 \& 2 & PUBLIC SERVICE OF OKLAHOMA & 1150 & FY-82 \\
ALLENS CREEK 1 & BWR & 150 & FY-82 \\
PEBBLE SPRINGS 1 \& 2 & POUSTON LIGHTING \& POWER & PWR & 1150 \\
SKAGIT/HANFORD 1 \& 2 & PORTLAND GENERAL ELECTRIC & 1260 & FY-83 \\
CLINCH RIVER & PUGET SOUND POWER \& LIGHT & PWR & 1277 & FY-83 \\
\hline
\end{tabular}

"CANCELLATION IS DEFINED AS PUBLIC ANNOUNCEMENT OF CANCELLATION OR WRITTEN NOTIFICATION TO NRC.

ONLY DOCKETED APPLICATIONS ARE INDICATED. 


\section{REACTOR CANCELLATIONS* \\ UNITS WITH CONSTRUCTION PERMITS}

\begin{tabular}{|c|c|c|c|c|}
\hline PLANT & UTILITY & TYPE & $\begin{array}{c}\text { MWE/PER } \\
\text { UNIT }\end{array}$ & DATE \\
\hline 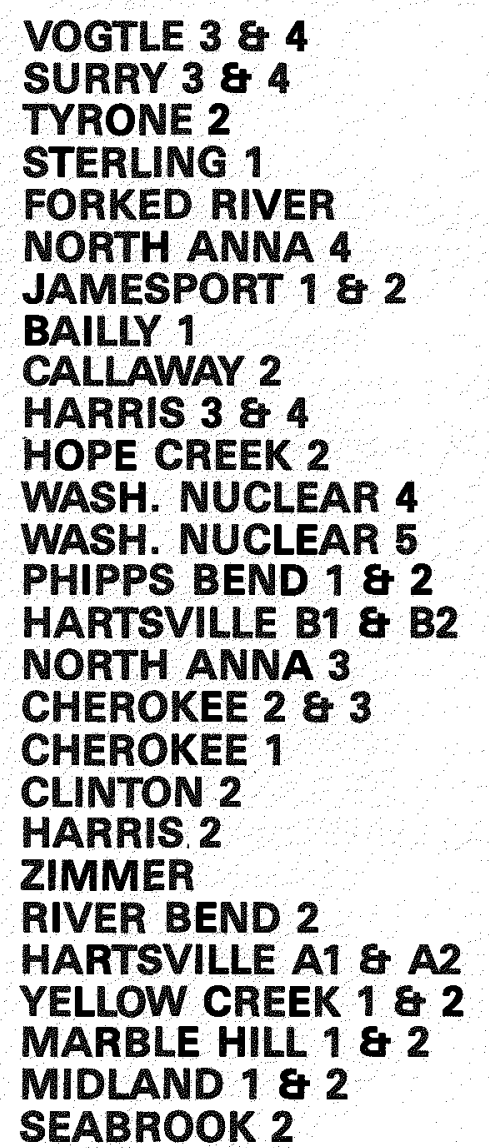 & $\begin{array}{l}\text { GEORGIA POWER } \\
\text { VIRGINIA ELECTRIC \& POWER } \\
\text { NORTHERN STATES POWER } \\
\text { ROCHESTER GAS \& ELECTRIC CORP. } \\
\text { JERSEY CENTRAL POWER \& LIGHT } \\
\text { VIRGINIA ELECTRIC \& POWER CO. } \\
\text { LONG ISLAND LIGHTING CO. } \\
\text { NORTHERN INDIANA PUBLIC SERVICE } \\
\text { UNION ELECTRIC } \\
\text { CAROLINA POWER \& LIGHT } \\
\text { PUBLIC SERVICE ELECTRIC \& GAS } \\
\text { WASH. PUBLIC POWER SUPPLY SYSTEM } \\
\text { WASH. PUBLIC POWER SUPPLY SYSTEM } \\
\text { TENNESSEE VALLEY AUTHORITY } \\
\text { TENNESSEE VALLEY AUTHORITY } \\
\text { VIRGINIA ELECTRIC \& POWER CO. } \\
\text { DUKE POWER } \\
\text { DUKE POWER } \\
\text { ILLINOIS POWER } \\
\text { CAROLINA POWER \& LIGHT } \\
\text { CINCINNATI GAS \& ELECTRIC } \\
\text { GULF STATES UTILITIES } \\
\text { TENNESSEE VALLEY AUTHORITY } \\
\text { TENNESSEE VALLEY AUTHORITY } \\
\text { PUBLIC SERVICE OF INDIANA } \\
\text { CONSUMERS POWER CORPORATION } \\
\text { PUBLIC SERVICE CO. OF NEW HAMPSHIRE }\end{array}$ & $\begin{array}{l}\text { PWR } \\
\text { PWR } \\
\text { PWR } \\
\text { PWR } \\
\text { PWR } \\
\text { PWR } \\
\text { PWR } \\
\text { BWR } \\
\text { PWR } \\
\text { PWR } \\
\text { BWR } \\
\text { PWR } \\
\text { PWR } \\
\text { BWR } \\
\text { BWR } \\
\text { PWR } \\
\text { PWR } \\
\text { PWR } \\
\text { BWR } \\
\text { PWR } \\
\text { BWR } \\
\text { BWR } \\
\text { BWR } \\
\text { BWR } \\
\text { PWR } \\
\text { PWR } \\
\text { PWR }\end{array}$ & $\begin{array}{r}1150 \\
882 \\
1150 \\
1150 \\
1070 \\
907 \\
1150 \\
660 \\
1150 \\
915 \\
1067 \\
1267 \\
1242 \\
1220 \\
1205 \\
907 \\
1280 \\
1280 \\
950 \\
915 \\
810 \\
934 \\
1205 \\
1285 \\
1130 \\
655 \\
1198\end{array}$ & $\begin{array}{c}\text { FY-75 } \\
\text { FY-7 } \\
\text { FY-79 } \\
\text { FY-80 } \\
\text { FY-81 } \\
\text { FY-81 } \\
\text { FY-81 } \\
\text { FY-81 } \\
\text { FY-82 } \\
\text { FY-82 } \\
\text { FY-82 } \\
\text { FY-82 } \\
\text { FY-82 } \\
\text { FY-82 } \\
\text { FY-82 } \\
\text { FY-83 } \\
\text { FY-83 } \\
\text { FY-83 } \\
\text { FY-84 } \\
\text { FY-84 } \\
\text { FY-84 } \\
\text { FY-84 } \\
\text { FY-84 } \\
\text { FY-84 } \\
\text { FY-85 } \\
\text { FY-86 } \\
\text { FY-89 }\end{array}$ \\
\hline
\end{tabular}

* CANCELLATION IS DEFINED AS PUBLIC ANNOUNCEMENT OF CANCELLATION OR WRITTEN NOTIFICATION TO NRC. ONLY DOCKETED APPLICATIONS ARE INDICATED. PRIOR TO FY-75 NO UNITS WERE CANCELLED WHICH HAD RECEIVED CONSTRUCTION PERMITS.

SOURCE: NRC 1/31/89

Part II: Section 3

Page 80 


\section{NUCLEAR POWER REACTORS BY LICENSEE}

\begin{tabular}{|c|c|c|c|}
\hline UTILITY & PLANT & UTILITY & PLANT \\
\hline $\begin{array}{l}\text { Alabama Power Co. } \\
\text { Arizona Public Service } \\
\text { Arkansas Power \& Light } \\
\text { Baltimore Gas \& Elec } \\
\text { Boston Edison } \\
\text { Carolina Power \& Light } \\
\text { Carolina Power \& Light } \\
\text { Carolina Power \& Light } \\
\text { Cleveland Electric Illuminating } \\
\text { Commonwealth Edison } \\
\text { Commonwealth Edison } \\
\text { Commonwealth Edison } \\
\text { Commonwealth Edison } \\
\text { Commonwealth Edison } \\
\text { Commonwealth Edison } \\
\text { Connecticut Yankee Atomic Power } \\
\text { Consolidated Edison } \\
\text { Consumers Power } \\
\text { Consumers Power } \\
\text { Detroit Edison } \\
\text { Duke Power } \\
\text { Duke Power } \\
\text { Duke Power } \\
\text { Duquesne Light } \\
\text { Florida Power \& Light } \\
\text { Florida Power \& Light }\end{array}$ & 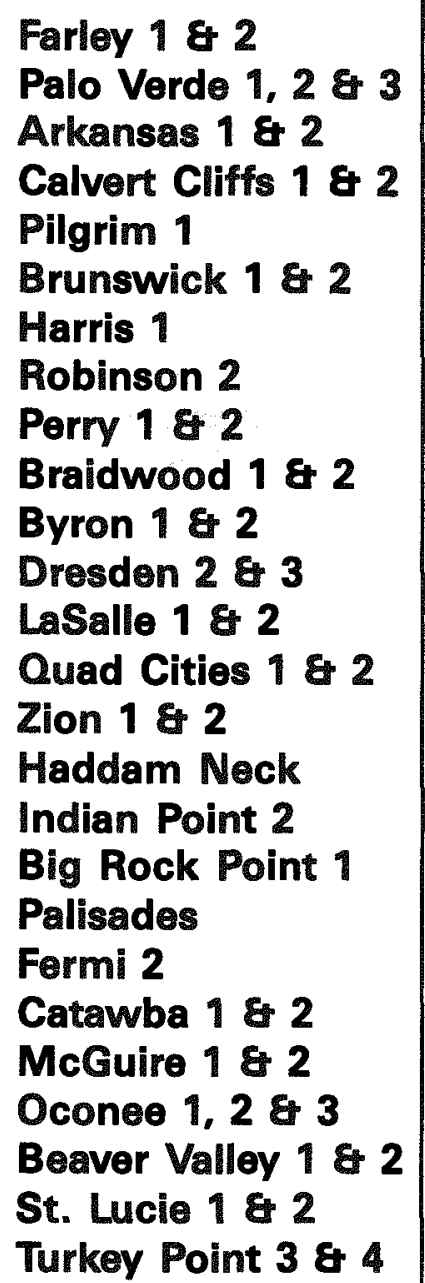 & $\begin{array}{l}\text { Florida Power Corporation } \\
\text { Georgia Power } \\
\text { Georgia Power } \\
\text { GPU Nuclear Corporation } \\
\text { GPU Nuclear Corporation } \\
\text { Gulf States Utilities } \\
\text { Houston Lighting \& Power } \\
\text { Company } \\
\text { Illinois Power } \\
\text { Indiana \& Michigan Electric } \\
\text { lowa Electric Power \& Light } \\
\text { Long Island Lighting Co. } \\
\text { Louisiana Power \& Light } \\
\text { Maine Yankee Atomic Power } \\
\text { Nebraska Public Power District } \\
\text { Niagara Mohawk Power Corp. } \\
\text { Northeast Nuclear Energy Co. } \\
\text { Northern States Power } \\
\text { Northern States Power } \\
\text { Omaha Public Power District } \\
\text { Pacific Gas \& Electric } \\
\text { Pennsylvania Power \& Light } \\
\text { Philadelphia Electric } \\
\text { Philadelphia Electric } \\
\text { Portland General Electric } \\
\text { New York Power Authority }\end{array}$ & $\begin{array}{l}\text { Crystal River } 3 \\
\text { Hatch } 1 \text { \& } 2 \\
\text { Vogtle } 1 \& 2 \\
\text { Three Mile Island } 1 \\
\text { Oyster Creok } 1 \\
\text { River Bend } 1 \\
\text { South Texas } 1 \& 2 \\
\\
\text { Clinton } 1 \\
\text { Cook } 1 \& 2 \\
\text { Duane Arnold } \\
\text { Shoreham } \\
\text { Waterford } 3 \\
\text { Maine Yankee } \\
\text { Cooper Station } \\
\text { Nine Mile Point } 1 \& 2 \\
\text { Millstone } 1,2 \text { \& } 3 \\
\text { Monticello } \\
\text { Prairie Island } 1 \& 2 \\
\text { Fort Calhoun } 1 \\
\text { Diablo Canyon } 1 \& 2 \\
\text { Susquehanna } 1 \& 2 \\
\text { Limerick } 1 \text { \& } 2 \\
\text { Peach Bottom } 2 \text { \& } 3 \\
\text { Trojan } \\
\text { Fitzpatrick }\end{array}$ \\
\hline
\end{tabular}


WO NOT MOROFLM
THY PAGE

Page 81 


\section{NUCLEAR POWER REACTORS BY LICENSEE}

\begin{tabular}{|c|c|c|c|}
\hline UTILITY & PLANT & UTILITY & PLANT \\
\hline 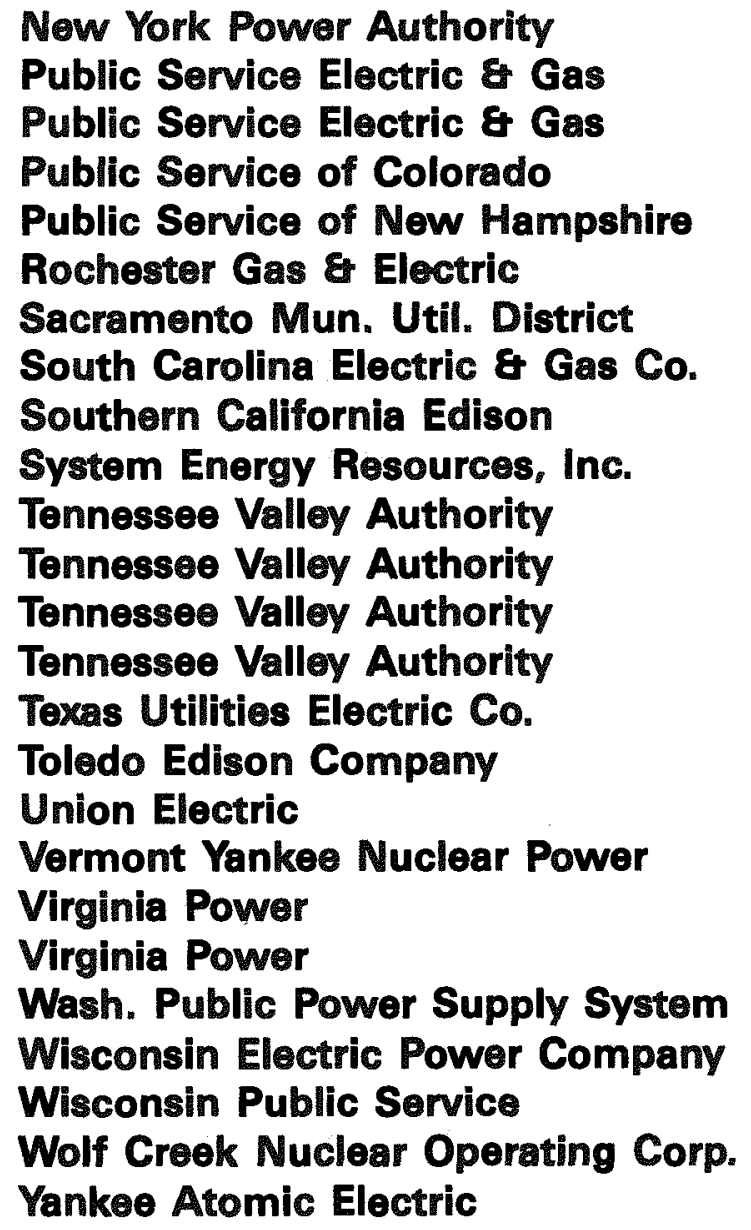 & $\begin{array}{l}\text { Indian Point } 3 \\
\text { Hope Creek } 1 \\
\text { Salem } 1 \text { \& } 2 \\
\text { Fort St. Vrain } \\
\text { Seabrook } 1 \\
\text { Ginna } \\
\text { Rancho Seco } 1 \\
\text { Summer } 1 \\
\text { San Onofre } 1,2 \text { \& } 3 \\
\text { Grand Gulf } 1 \& 2 \\
\text { Bellefonte } 1 \& \text { \& } 2 \\
\text { Browns Ferry } 1,2 \text { \& } 3 \\
\text { Sequoyah } 1 \text { \& } 2 \\
\text { Watts Bar } 1 \& 2 \\
\text { Comanche Peak } 1 \& 2 \\
\text { Davis-Besse } 1 \\
\text { Callaway } 1 \\
\text { Vermont Yankee } 1 \\
\text { North Anna } 1 \& \text { \& } 2 \\
\text { Surry } 1 \& 2 \\
\text { WNP 1, } 2 \text { \& } 3 \\
\text { Point Beach } 1 \& 2 \\
\text { Kewaunee } \\
\text { Wolf Creek } 1 \\
\text { Yankee Rowe } 1\end{array}$ & & \\
\hline
\end{tabular}




\section{WORLD LIST OF NUCLEAR POWER REACTORS}

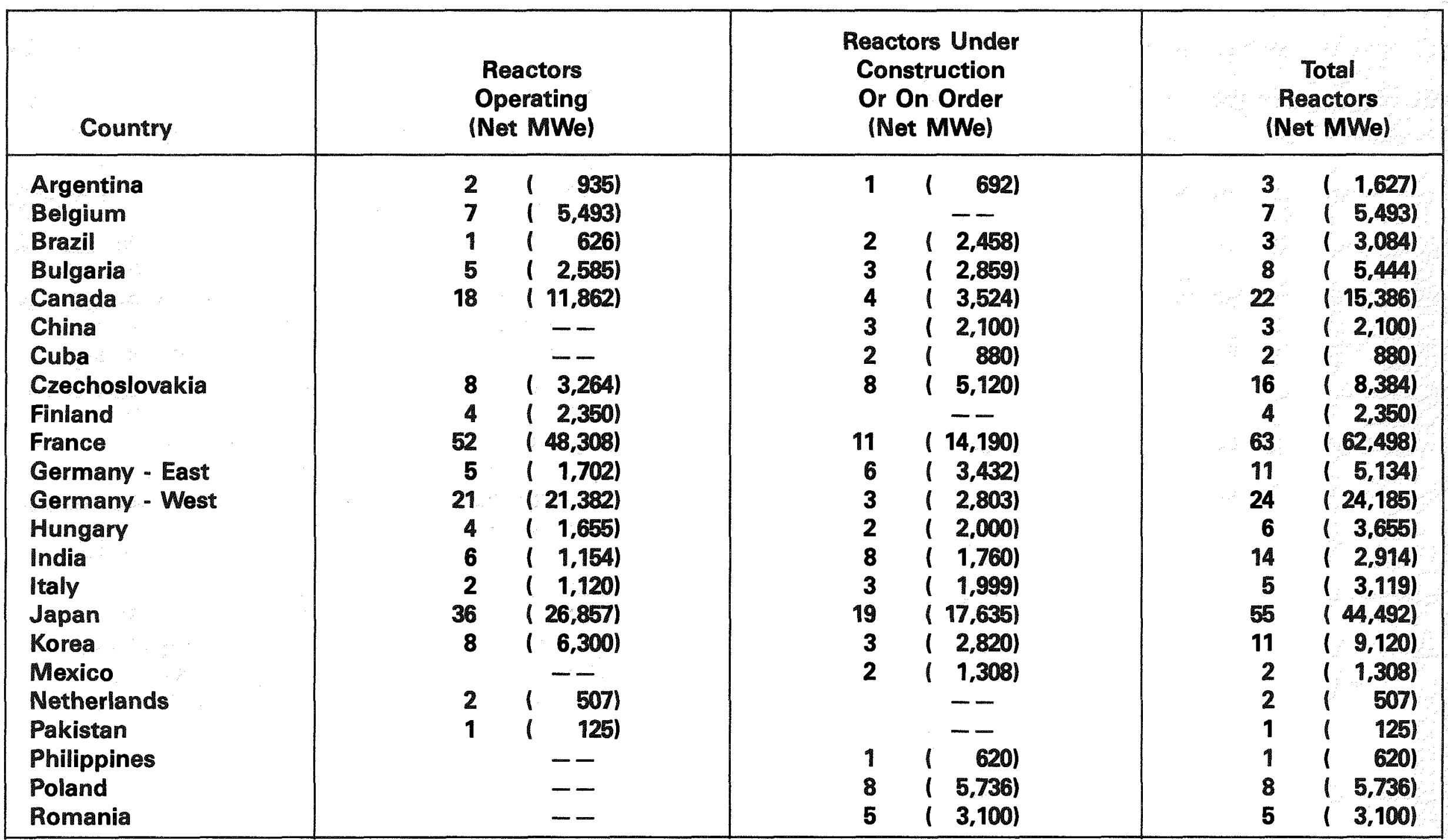




\section{WORLD LIST OF NUCLEAR POWER REACTORS}

\begin{tabular}{|c|c|c|c|c|c|c|}
\hline \multirow{2}{*}{ Country } & \multicolumn{2}{|c|}{$\begin{array}{l}\text { Reactors } \\
\text { Operating } \\
\text { (Net MWe) }\end{array}$} & \multicolumn{2}{|c|}{$\begin{array}{l}\text { Reactors Under } \\
\text { Construction } \\
\text { Or On Order } \\
\text { (Net MWe) }\end{array}$} & \multicolumn{2}{|c|}{$\begin{array}{c}\text { Total } \\
\text { Reactors } \\
\text { (Net MWe) }\end{array}$} \\
\hline & & $(1,840)$ & & -- & 2 & $(1,840)$ \\
\hline Spain & & $(7,526)$ & & ( 6,822) & 17 & $(14,348)$ \\
\hline Sweden & 12 & ( 9,667$)$ & & -- & 12 & $(9,667)$ \\
\hline Switzerland & 5 & ( 2,951) & & -- & 5 & ( 2,951) \\
\hline Taiwan & 6 & ( 4,884$)$ & & -- & 6 & ( 4,884) \\
\hline United Kingdom & 41 & $(13,468)$ & 2 & $(1,875)$ & 43 & ( 15,343$)$ \\
\hline USA* & 111 & $(98,000)$ & 12 & $(14,000)$ & 123 & $(112,000)$ \\
\hline USSR & 50 & $(36,253)$ & 23 & $(22,703)$ & 73 & $(58,956)$ \\
\hline Yugoslavia & 1 & ( 620$)$ & - & - & 1 & 16201 \\
\hline TOTAL & $\overline{420}$ & $(311,434)$ & 138 & $(120,436)$ & $\overline{558}$ & $(431,870)$ \\
\hline
\end{tabular}

* For USA Data NRC 2/89 
NOTES

Page 86$$
\Longrightarrow
$$ 


\section{NOTES}

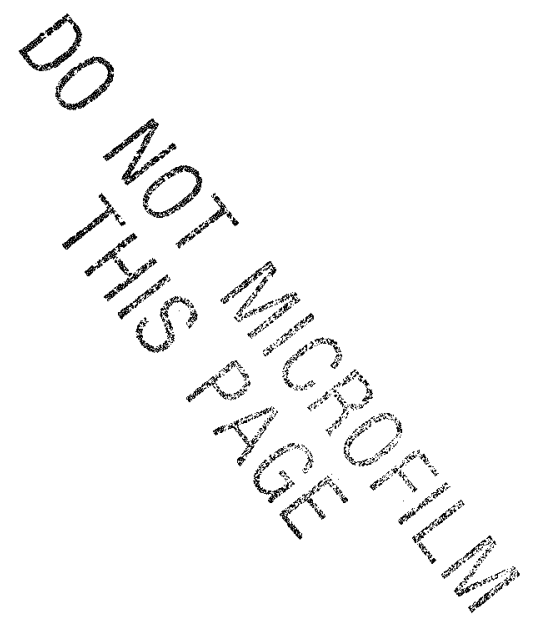

Page 87 


\section{NOTES}

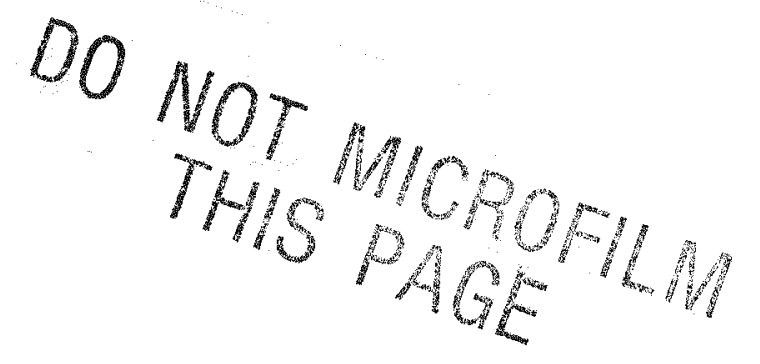




\section{NOTES}

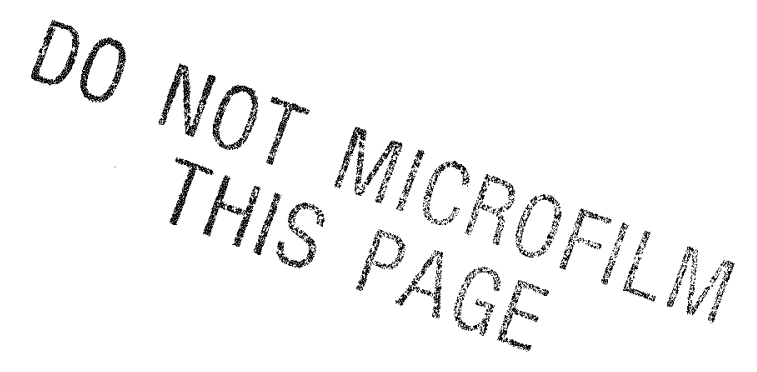

